Switchable and scalable heteroarylation of primary amines with 2-chlorobenzothiazoles under transition-metal-free and solvent-free conditions

Hua Cheng, ${ }^{\text {a }}$ ‡ Yan-Qiu Zhu, ${ }^{\text {a, } ~}$ Peng-Fei Liu, ${ }^{a}$ Kai-Qiang Yang, ${ }^{a}$ Jin Yan, ${ }^{a}$ Wei Sang, Xiao-Sheng Tang, ${ }^{\mathrm{c}, \mathrm{d}}$ Rui Zhang, ${ }^{* \mathrm{a}}$ and Cheng Chen ${ }^{* b}$

${ }^{\text {a }}$ Department of Chemical Engineering and Food Science, Hubei University of Arts and Science, 296 Longzhong Road, Xiangyang 441053, P. R. China.

b State Key Laboratory of Advanced Technology for Materials Synthesis and Processing, Wuhan University of Technology, 122 Luoshi Road, Wuhan 430070, P. R. China.

${ }^{c}$ College of Optoelectronic Engineering, Chongqing University of Posts and Telecommunications, Chongqing 400065, China.

${ }^{\mathrm{d}}$ School of Materials Science and Engineering, Zhengzhou University, Zhengzhou 450001, China.

‡ These authors contributed equally to this work.

\title{
Corresponding Authors
}

*E-mail for Dr. Rui Zhang: rzhangccnu@163.com; *E-mail for Dr. Cheng Chen: chengchen@whut.edu.cn.

\section{Contents:}

1. Supplementary results

2. Green metrics of all target compounds 


\section{Supplementary results}

Table S1. Optimization of reaction conditions ${ }^{a}$

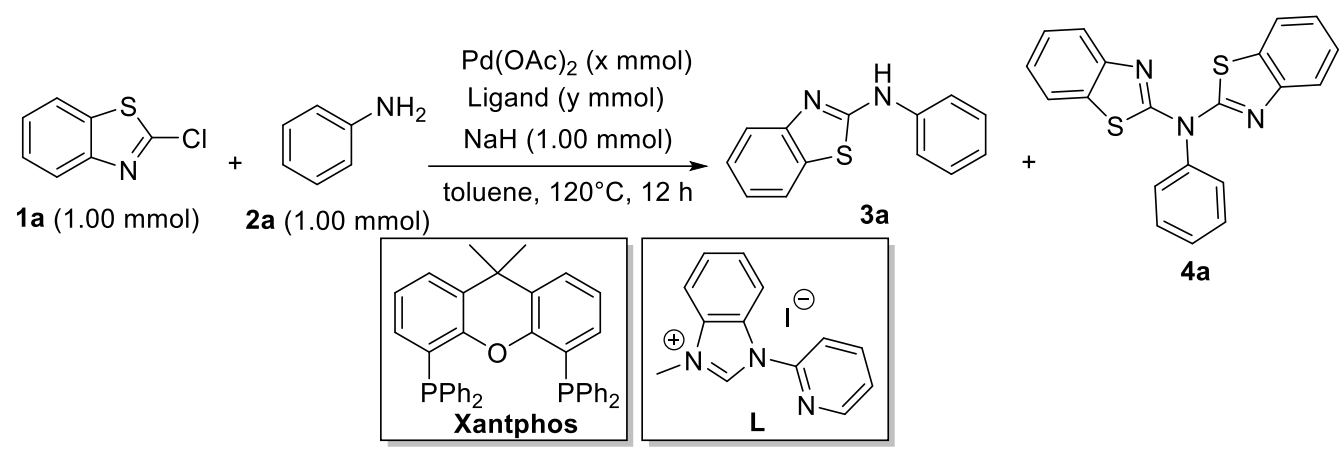

\begin{tabular}{|c|c|c|c|c|c|}
\hline \multirow{2}{*}{ Entry } & \multirow{2}{*}{$\mathrm{x}$} & \multirow{2}{*}{ Ligand (y) } & \multicolumn{3}{|c|}{ Yield $(\%)^{a}$} \\
\hline & & & $3 \mathbf{a}$ & $4 a$ & Unreacted 1a \\
\hline 1 & 0.025 & Xantphos (0.050) & 92 & 5 & - \\
\hline 2 & 0.025 & $\mathbf{L}(0.025)$ & - & 94 & - \\
\hline 3 & 0.025 & - & 5 & 51 & 15 \\
\hline
\end{tabular}

${ }^{a}$ This detailed procedure can be found in the literature ${ }^{[3]} ;{ }^{b} \mathrm{NMR}$ yield using 1,3,5-trimethoxybenzene as an internal standard (average of two consistent runs).

Table S2. The reactions of ethyl 2-chlorobenzo[d]thiazole-6-carboxylate (1w) and aniline (2a) under the base-free condition ${ }^{a}$

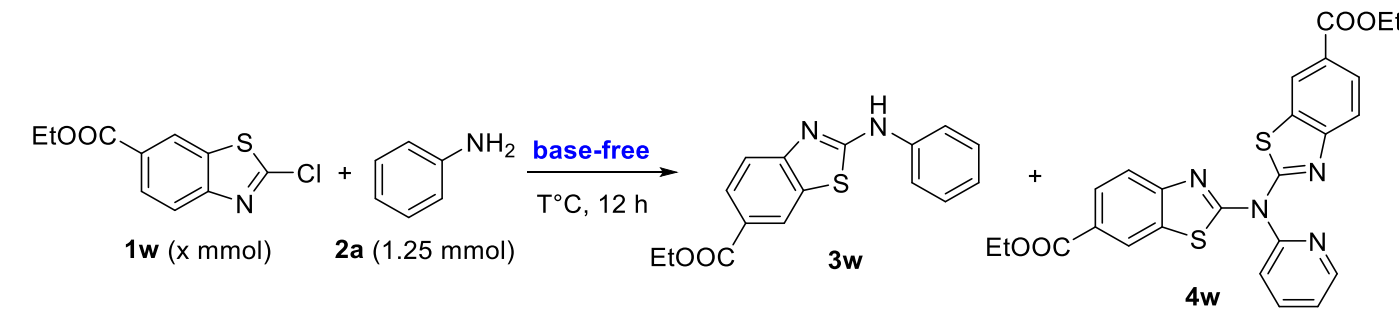

\begin{tabular}{ccccc}
\hline \multirow{2}{*}{ Entry } & $\mathrm{x}$ & $\mathrm{T}$ & \multicolumn{2}{c}{${\text { Yields }(\%)^{a}}$} \\
\cline { 4 - 5 } & & & $\mathbf{3 w}$ & $\mathbf{4 w}$ \\
\hline 1 & 1.00 & 100 & 93 & - \\
3 & 2.00 & 100 & 92 & - \\
\hline
\end{tabular}

${ }^{a}$ NMR yield using 1,3,5-trimethoxybenzene as an internal standard (average of two consistent runs). 


\section{Green metrics of all target compounds}

Table S3. The calculated green metrics for compounds 3 .

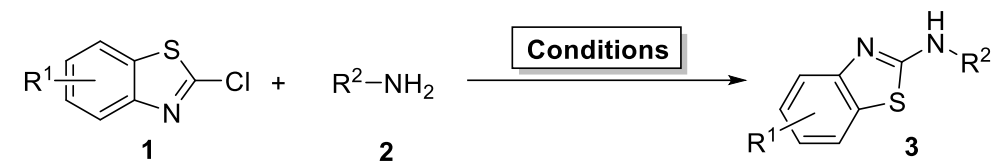

\begin{tabular}{|c|c|c|c|c|c|c|c|}
\hline Ref. & $\begin{array}{c}\text { Compound } \\
\text { No. }\end{array}$ & $\begin{array}{l}\mathrm{AE} \\
(\%) \\
\end{array}$ & $\begin{array}{l}\text { E-factor } \\
(\mathrm{kg} / \mathrm{kg})\end{array}$ & $\begin{array}{l}\text { CE } \\
(\%)\end{array}$ & $\begin{array}{c}\text { RME } \\
(\%)\end{array}$ & $\begin{array}{c}\text { MI } \\
(\mathrm{kg} / \mathrm{kg})\end{array}$ & $\begin{array}{l}\text { MP } \\
(\%) \\
\end{array}$ \\
\hline This work $^{a}$ & $\mathbf{3 a}$ & 86.1 & 0.4 & 82.5 & 72.8 & 1.4 & 72.8 \\
\hline [1] & $3 \mathbf{a}$ & 86.1 & 1.1 & 51.3 & 47.7 & 2.1 & 47.7 \\
\hline$[2]^{b}$ & 3a & 86.1 & 19.9 & 63.6 & 59.1 & 20.9 & 4.8 \\
\hline$[2]^{c}$ & 3a & 86.1 & 8.1 & 26.0 & 25.9 & 9.1 & 11.0 \\
\hline$[3]^{d}$ & $3 a$ & 86.1 & 11.9 & 86.0 & 74.1 & 12.9 & 7.8 \\
\hline This work $^{a}$ & $3 b$ & 86.8 & 0.4 & 80.0 & 71.2 & 1.4 & 71.2 \\
\hline [1] & $3 \mathbf{b}$ & 86.8 & 1.0 & 53.3 & 50.1 & 2.0 & 50.1 \\
\hline$[3]^{d}$ & $3 \mathbf{b}$ & 86.8 & 10.9 & 88.0 & 76.4 & 11.9 & 8.4 \\
\hline This work $^{a}$ & $3 c$ & 87.5 & 0.4 & 82.7 & 73.7 & 1.4 & 73.7 \\
\hline This work $^{a}$ & 3d & 88.0 & 0.4 & 80.7 & 72.9 & 1.4 & 72.9 \\
\hline This work $^{a}$ & $3 e$ & 88.6 & 0.4 & 79.3 & 72.2 & 1.4 & 72.2 \\
\hline$[3]^{d}$ & $3 e$ & 88.6 & 9.7 & 85.0 & 75.3 & 10.7 & 9.4 \\
\hline This work $^{a}$ & $3 f$ & 87.8 & 0.5 & 75.0 & 67.4 & 1.5 & 67.4 \\
\hline This work $^{a}$ & $3 g$ & 90.6 & 0.9 & 58.3 & 51.6 & 1.9 & 51.6 \\
\hline This work $^{a}$ & $3 h$ & 89.3 & 0.6 & 72.6 & 64.3 & 1.6 & 64.3 \\
\hline This work $^{a}$ & $3 \mathbf{i}$ & 87.7 & 0.6 & 71.7 & 63.4 & 1.6 & 63.4 \\
\hline [1] & $3 \mathbf{i}$ & 87.7 & 1.3 & 47.9 & 43.0 & 2.3 & 43.0 \\
\hline This work $^{a}$ & $3 \mathbf{j}$ & 87.0 & 0.5 & 73.5 & 64.9 & 1.5 & 64.9 \\
\hline [1] & $3 \mathbf{j}$ & 87.0 & 1.3 & 47.9 & 43.6 & 2.3 & 43.6 \\
\hline$[3]^{d}$ & $\mathbf{3 j}$ & 87.0 & 11.9 & 80.0 & 69.6 & 12.9 & 7.8 \\
\hline This work $^{a}$ & $3 k$ & 89.5 & 0.5 & 75.6 & 67.4 & 1.5 & 67.4 \\
\hline This work $^{a}$ & 31 & 89.0 & 1.0 & 55.1 & 49.2 & 2.0 & 49.2 \\
\hline$[3]^{d}$ & 31 & 89.0 & 14.6 & 56.0 & 49.8 & 15.6 & 6.4 \\
\hline This work $^{a}$ & $3 \mathrm{~m}$ & 87.3 & 1.8 & 40.0 & 35.6 & 2.8 & 35.6 \\
\hline This work $^{a}$ & $3 n$ & 88.1 & 0.5 & 76.2 & 67.4 & 1.5 & 67.4 \\
\hline This work $^{e}$ & $3 n$ & 88.1 & 2.2 & 41.5 & 36.2 & 3.2 & 31.7 \\
\hline This work $^{a}$ & 30 & 89.2 & 1.3 & 48.4 & 44.4 & 2.3 & 44.4 \\
\hline This work $^{a}$ & $3 p$ & 89.7 & 0.4 & 75.1 & 69.0 & 1.4 & 69.0 \\
\hline$[3]^{d}$ & $3 p$ & 89.7 & 8.3 & 88.0 & 79.0 & 9.3 & 10.8 \\
\hline This work $^{a}$ & $3 q$ & 89.7 & 0.8 & 62.2 & 57.1 & 1.8 & 57.1 \\
\hline$[3]^{d}$ & $3 q$ & 89.7 & 14.7 & 52.0 & 46.7 & 15.7 & 6.4 \\
\hline This work $^{a}$ & $3 r$ & 86.8 & 0.4 & 80.9 & 72.0 & 1.4 & 72.0 \\
\hline$[3]^{d}$ & $3 r$ & 86.8 & 11.9 & 81.0 & 70.3 & 12.9 & 7.7 \\
\hline
\end{tabular}




\begin{tabular}{|c|c|c|c|c|c|c|c|}
\hline This work $^{a}$ & $3 s$ & 86.8 & 0.5 & 74.7 & 66.5 & 1.5 & 66.5 \\
\hline$[3]^{d}$ & $3 s$ & 86.8 & 18.0 & 55.0 & 47.8 & 19.0 & 5.3 \\
\hline This work $^{a}$ & $3 t$ & 86.8 & 0.4 & 82.2 & 72.9 & 1.4 & 72.9 \\
\hline$[3]^{d}$ & $3 t$ & 86.8 & 10.5 & 91.0 & 79.0 & 11.5 & 8.7 \\
\hline This work $^{a}$ & $3 \mathbf{u}$ & 87.5 & 0.4 & 81.3 & 73.0 & 1.4 & 73.0 \\
\hline$[3]^{d}$ & $3 \mathbf{u}$ & 87.5 & 11.0 & 82.0 & 71.8 & 12.0 & 8.3 \\
\hline This work $^{a}$ & $3 v$ & 87.7 & 1.2 & 50.2 & 45.6 & 2.2 & 45.6 \\
\hline$[3]^{d}$ & $3 v$ & 87.7 & 11.6 & 77.0 & 67.6 & 12.6 & 7.9 \\
\hline This work $^{a}$ & $3 w$ & 89.1 & 0.3 & 85.0 & 77.5 & 1.3 & 77.5 \\
\hline 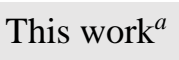 & $3 x$ & 86.8 & 0.7 & 62.0 & 58.2 & 1.7 & 58.2 \\
\hline [1] & $3 x$ & 86.8 & 1.2 & 48.0 & 45.1 & 2.2 & 45.1 \\
\hline$[2]^{b}$ & $3 x$ & 86.8 & 42.9 & 28.0 & 26.3 & 43.9 & 2.3 \\
\hline This work $^{a}$ & $3 y$ & 87.5 & 0.8 & 59.3 & 56.2 & 1.8 & 56.2 \\
\hline This work $^{a}$ & $3 z$ & 86.5 & 0.7 & 62.9 & 58.0 & 1.7 & 58.0 \\
\hline This work $^{a}$ & $3 \mathbf{a}^{\prime}$ & 86.2 & 4.1 & 22.6 & 19.8 & 5.1 & 19.8 \\
\hline This work $^{e}$ & $3 \mathbf{a}^{\prime}$ & 86.2 & 2.0 & 40.0 & 32.9 & 3.0 & 32.9 \\
\hline$[3]^{d}$ & $3 \mathbf{a}^{\prime}$ & 86.2 & 25.9 & 41.0 & 35.3 & 26.9 & 3.7 \\
\hline$[3]^{f}$ & $3 \mathbf{a}^{\prime}$ & 86.2 & 14.6 & 70.0 & 60.3 & 15.6 & 6.4 \\
\hline This work $^{a}$ & $3 b^{\prime}$ & 88.8 & 1.0 & 54.7 & 50.3 & 2.0 & 50.3 \\
\hline
\end{tabular}

${ }^{a}$ Under the base-free condition, ${ }^{b}$ under the Cu-catalyzed condition, ${ }^{c}$ under the Pd-catalyzed condition, ${ }^{d}$ via the $\mathrm{Pd} / \mathrm{Xantphos}$ catalytic system, ${ }^{e}$ under the $\mathrm{NaH}$-promoted condition, ${ }^{f}$ via the $\mathrm{Pd} / \mathrm{NHC}$ catalytic system.

Table S4. The calculated green metrics for compounds 4 .

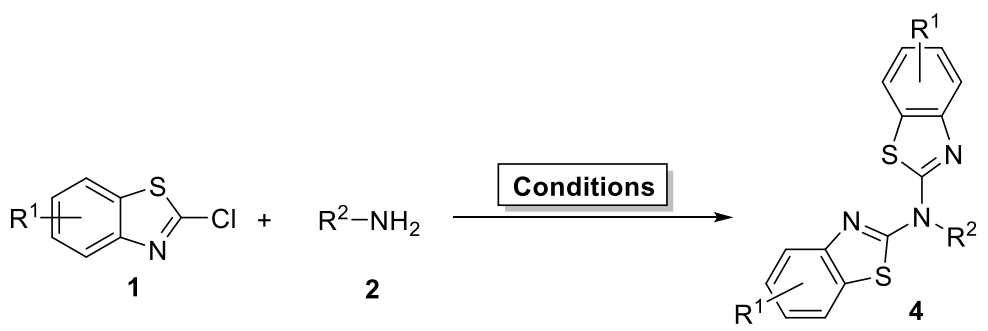

\begin{tabular}{cccccccc}
\hline Ref. & $\begin{array}{c}\text { Compound } \\
\text { No. }\end{array}$ & $\begin{array}{c}\text { AE } \\
(\%)\end{array}$ & $\begin{array}{c}\text { E-factor } \\
(\mathrm{kg} / \mathrm{kg})\end{array}$ & $\begin{array}{c}\text { CE } \\
(\%)\end{array}$ & $\begin{array}{c}\text { RME } \\
(\%)\end{array}$ & $\begin{array}{c}\text { MI } \\
(\mathrm{kg} / \mathrm{kg})\end{array}$ & $\begin{array}{c}\text { MP } \\
(\%)\end{array}$ \\
\hline This work $^{a}$ & $\mathbf{4 a}$ & 83.1 & 0.9 & 76.6 & 54.0 & 1.9 & 62.3 \\
{$[3]^{b}$} & $\mathbf{4 a}$ & 83.1 & 14.2 & 70.0 & 62.2 & 15.2 & 6.6 \\
This work $^{a}$ & $\mathbf{4 b}$ & 83.7 & 0.8 & 78.0 & 55.6 & 1.8 & 64.0 \\
{$[3]^{b}$} & $\mathbf{4 b}$ & 83.7 & 13.1 & 71.3 & 64.1 & 14.1 & 7.1 \\
This work $^{a}$ & $\mathbf{4 c}$ & 84.2 & 0.7 & 80.6 & 58.4 & 1.7 & 66.9 \\
This work $^{a}$ & $\mathbf{4 d}$ & 84.6 & 1.2 & 63.4 & 45.9 & 2.2 & 52.4 \\
This work $^{a}$ & $\mathbf{4 e}$ & 85.1 & 1.3 & 59.3 & 43.3 & 2.3 & 49.3 \\
{$[3]^{b}$} & $\mathbf{4 e}$ & 85.1 & 12.1 & 65.6 & 60.6 & 13.1 & 7.6 \\
This work $^{a}$ & $\mathbf{4 f}$ & 84.7 & 1.1 & 65.6 & 47.8 & 2.1 & 54.6 \\
This work $^{a}$ & $\mathbf{4 g}$ & 86.9 & 1.0 & 63.8 & 50.3 & 2.0 & 56.6 \\
This work $^{a}$ & $\mathbf{4 h}$ & 85.7 & 0.9 & 70.6 & 53.8 & 1.9 & 61.0
\end{tabular}




\begin{tabular}{|c|c|c|c|c|c|c|c|}
\hline This work $^{a}$ & $4 \mathbf{i}$ & 84.4 & 0.9 & 72.3 & 53.0 & 1.9 & 60.7 \\
\hline This work $^{a}$ & $4 j$ & 83.8 & 1.3 & 61.3 & 44.2 & 2.3 & 50.8 \\
\hline$[3]^{b}$ & $4 j$ & 83.8 & 13.7 & 69.2 & 60.5 & 14.7 & 6.8 \\
\hline This work $^{a}$ & $4 k$ & 85.9 & 1.0 & 65.1 & 49.5 & 2.0 & 56.1 \\
\hline This work $^{a}$ & 41 & 85.4 & 6.4 & 18.0 & 13.5 & 7.4 & 15.3 \\
\hline$[3]^{b}$ & 41 & 85.4 & 38.8 & 22.5 & 19.4 & 39.8 & 2.5 \\
\hline This work $^{a}$ & $4 m$ & 84.1 & 1.7 & 51.4 & 37.1 & 2.7 & 42.5 \\
\hline This work $^{a}$ & 40 & 85.7 & 1.5 & 53.8 & 39.4 & 2.5 & 44.8 \\
\hline This work $^{a}$ & $4 p$ & 86.1 & 0.7 & 77.6 & 57.7 & 1.7 & 65.2 \\
\hline$[3]^{b}$ & $4 p$ & 86.1 & 12.3 & 58.8 & 54.7 & 13.3 & 7.5 \\
\hline This work $^{a}$ & $4 q$ & 86.1 & 1.0 & 66.1 & 49.1 & 2.0 & 55.6 \\
\hline$[3]^{b}$ & $4 q$ & 86.1 & 42.9 & 17.8 & 16.6 & 43.9 & 2.3 \\
\hline This work $^{a}$ & $4 r$ & 83.7 & 0.9 & 73.7 & 52.5 & 1.9 & 60.5 \\
\hline$[3]^{b}$ & $4 r$ & 83.7 & 15.7 & 60.0 & 53.9 & 16.7 & 6.0 \\
\hline This work $^{a}$ & $4 s$ & 83.7 & 2.1 & 44.6 & 31.8 & 3.1 & 36.6 \\
\hline$[3]^{b}$ & $4 s$ & 83.7 & 20.6 & 46.5 & 41.8 & 21.6 & 4.6 \\
\hline This work $^{a}$ & $4 t$ & 84.2 & 1.6 & 52.5 & 38.0 & 2.6 & 43.5 \\
\hline$[3]^{b}$ & $4 t$ & 84.2 & 16.9 & 56.6 & 50.4 & 17.9 & 5.6 \\
\hline This work $^{a}$ & $4 u$ & 85.2 & 1.5 & 54.2 & 39.9 & 2.5 & 45.3 \\
\hline$[3]^{b}$ & $4 u$ & 85.2 & 20.4 & 44.0 & 40.1 & 21.4 & 4.7 \\
\hline This work $^{a}$ & $4 v$ & 85.5 & 2.1 & 43.4 & 32.0 & 3.1 & 36.2 \\
\hline$[3]^{b}$ & $4 v$ & 85.5 & 13.0 & 64.6 & 60.5 & 14.0 & 7.1 \\
\hline This work $^{a}$ & $4 w$ & 87.4 & 0.8 & 71.3 & 55.1 & 1.8 & 61.4 \\
\hline$[3]^{b}$ & $4 w$ & 87.4 & 12.5 & 60.9 & 56.4 & 13.5 & 7.4 \\
\hline$[3]^{c}$ & $4 w$ & 87.4 & 17.6 & 44.7 & 41.4 & 18.6 & 5.4 \\
\hline This work $^{a}$ & $4 x$ & 83.7 & 1.6 & 54.9 & 39.1 & 2.6 & 45.0 \\
\hline This work $^{a}$ & $4 y$ & 84.2 & 1.2 & 63.8 & 45.9 & 2.2 & 52.6 \\
\hline This work $^{a}$ & $4 z$ & 83.4 & 1.6 & 53.6 & 38.3 & 2.6 & 44.1 \\
\hline
\end{tabular}

${ }^{a}$ Under the NaH-promoted condition, ${ }^{b}$ via the $\mathrm{Pd} / \mathrm{NHC}$ catalytic system, ${ }^{c}$ via the $\mathrm{Pd} / \mathrm{Xantphos}$ catalytic system.

\section{References}

[1] H. F. Motiwala, R. Kumar and A. K. Chakraborti, Aust. J. Chem., 2007, 60, 369-374.

[2] S. Toulot, T. Heinrich and F. R. Leroux, Adv. Synth. Catal., 2013, 355, 3263-3272.

[3] Y. Q. Zhu, R. Zhang, W. Sang, H. J. Wang, Y. Wu, B. Y. Yu, J. C. Zhang, H.

Cheng and C. Chen, Org. Chem. Front., 2020, 7, 1981-1990. 


\section{$\underline{\text { 3. Original NMR spectra for compounds } 3 \text { and } 4}$}

${ }^{1} \mathrm{H}$ NMR spectrum for $\mathbf{3 a}$
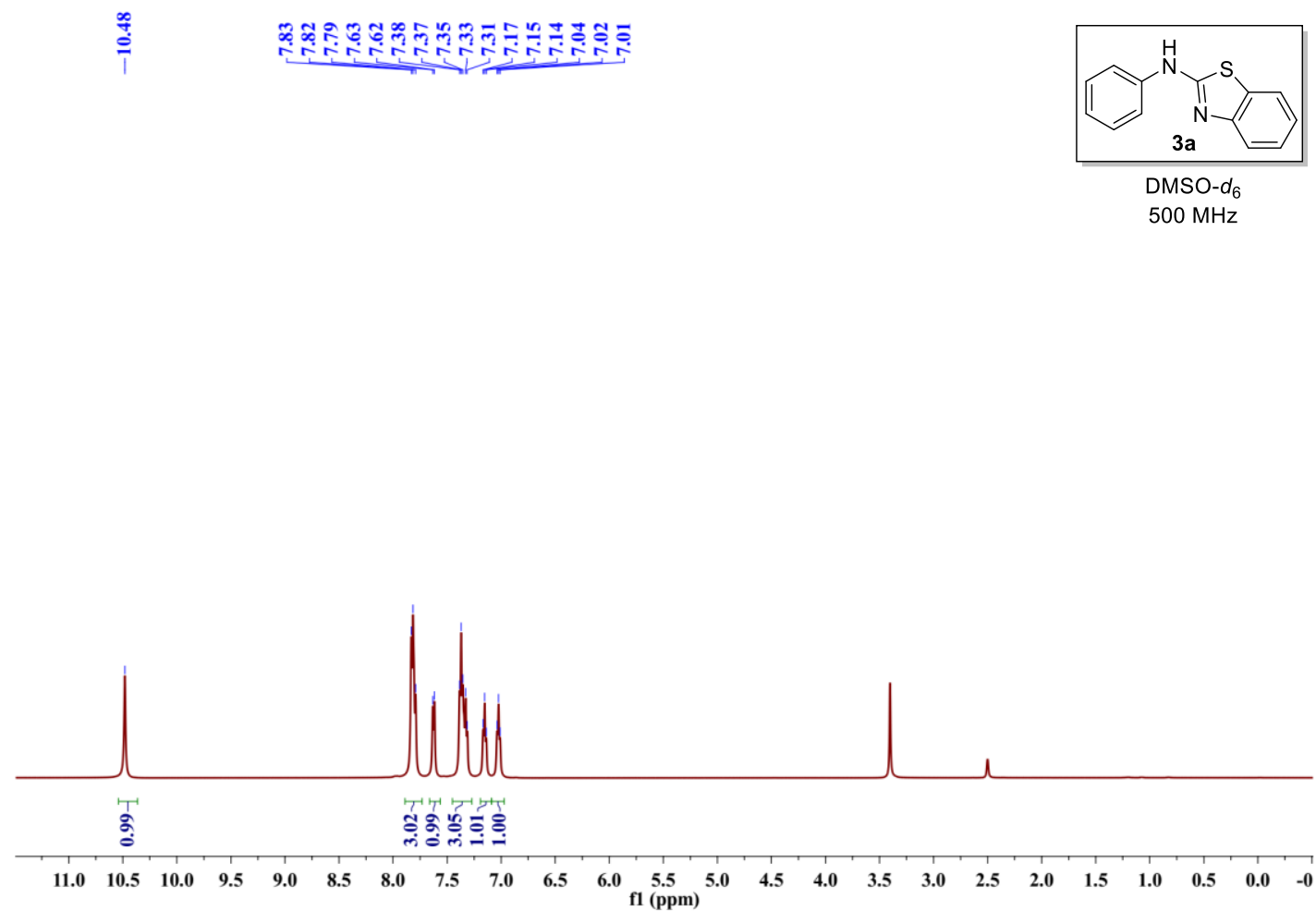

${ }^{13} \mathrm{C}$ NMR spectrum for $\mathbf{3 a}$

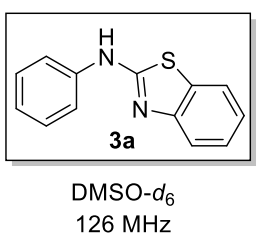

ๆ

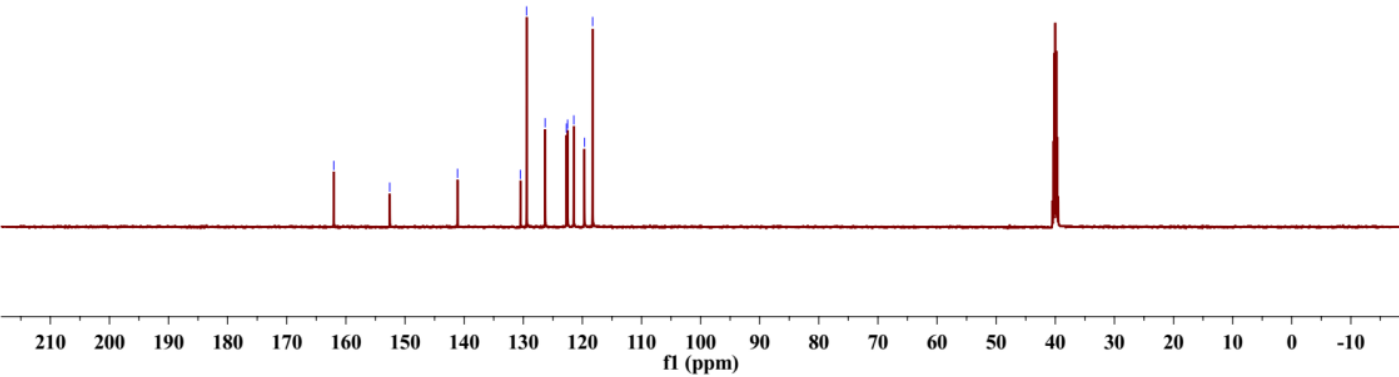


${ }^{1} \mathrm{H}$ NMR spectrum for $\mathbf{3 b}$

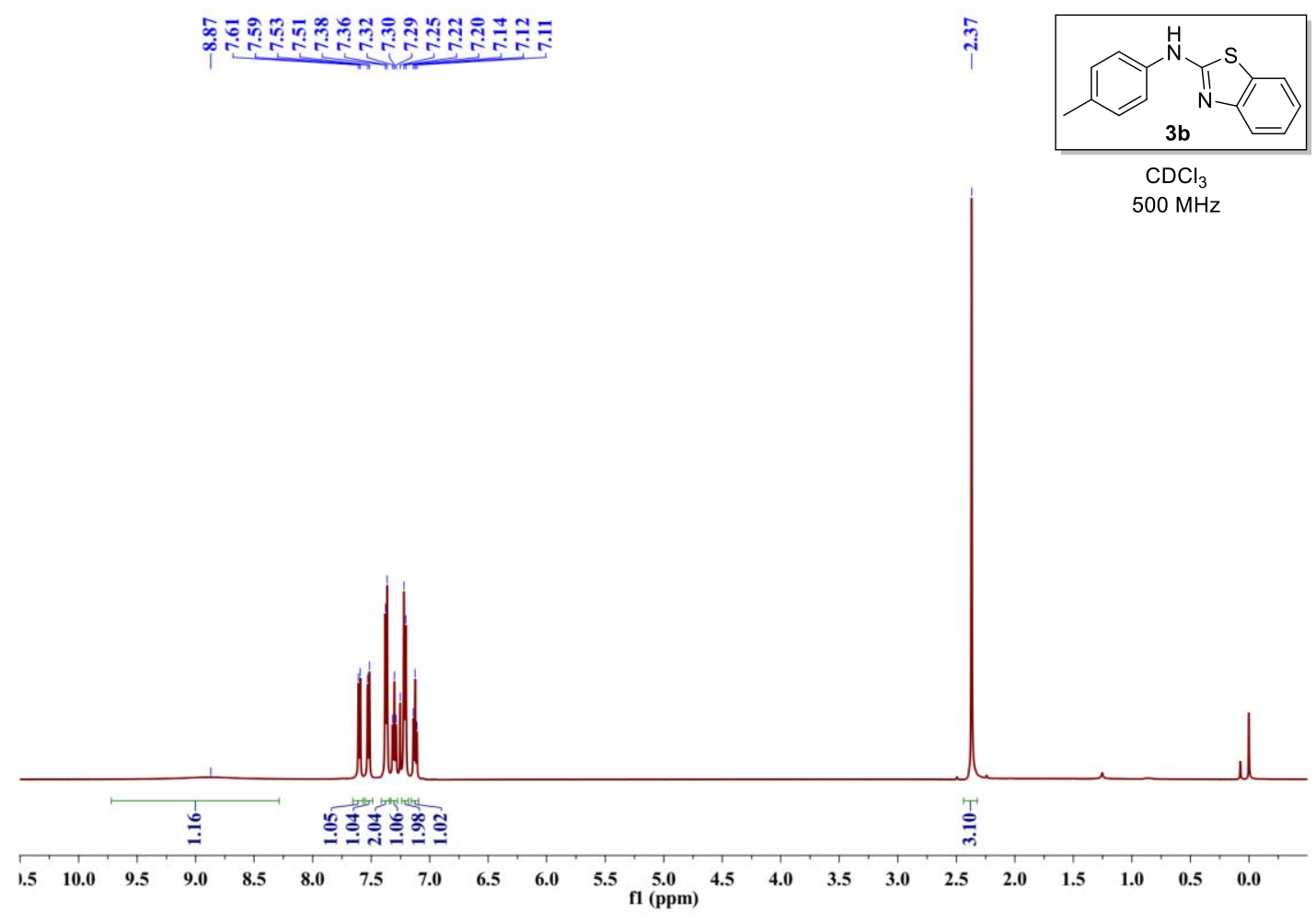

$>{ }^{13} \mathrm{C}$ NMR spectrum for $\mathbf{3 b}$
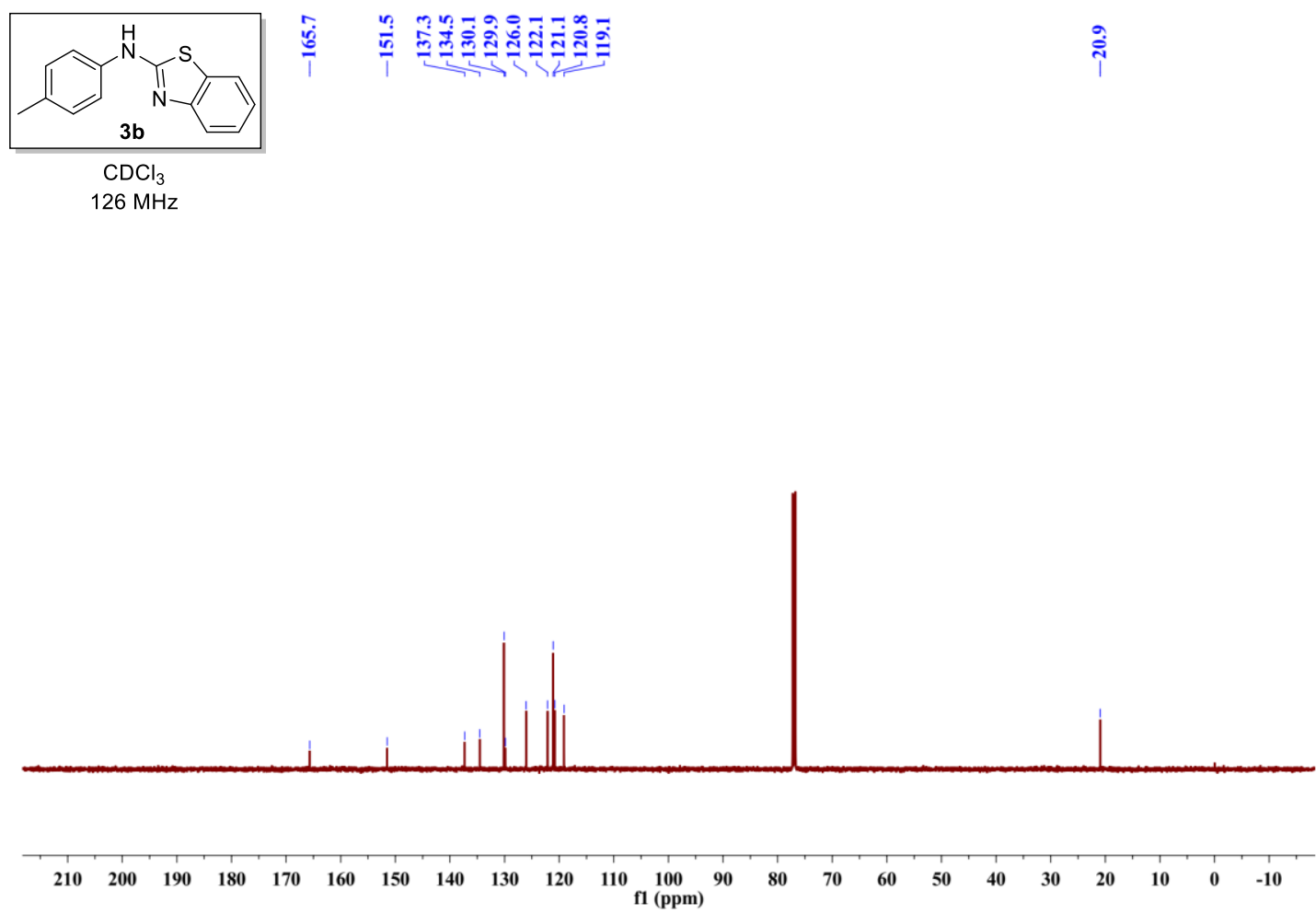
${ }^{1} \mathrm{H}$ NMR spectrum for $\mathbf{3 c}$

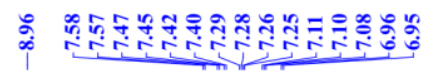

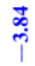
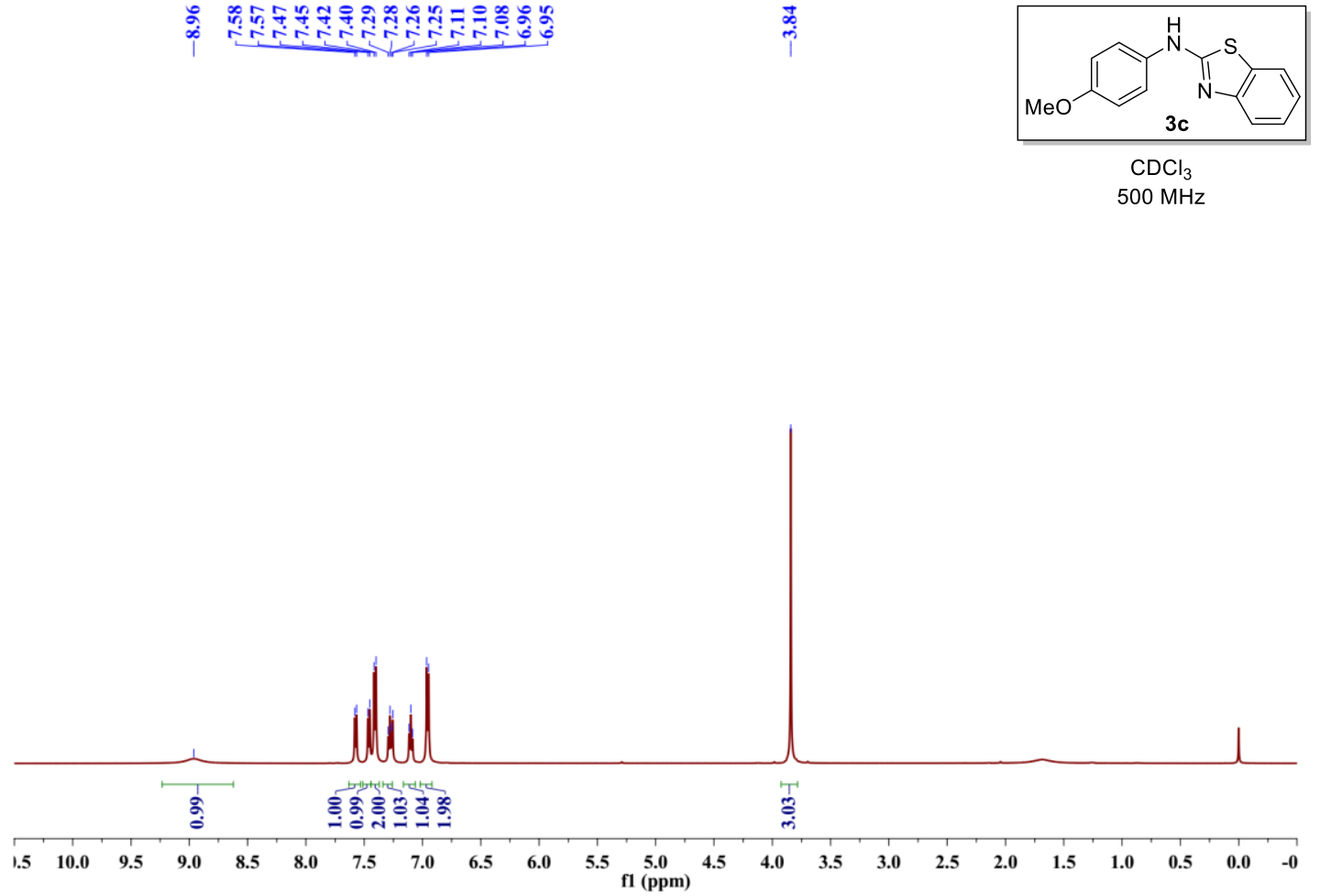

$>{ }^{13} \mathrm{C}$ NMR spectrum for $3 \mathrm{c}$

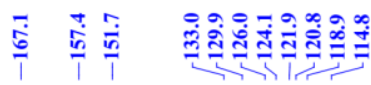

if

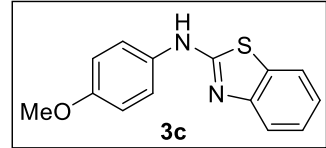

$\mathrm{CDCl}_{3}$

$126 \mathrm{MHz}$

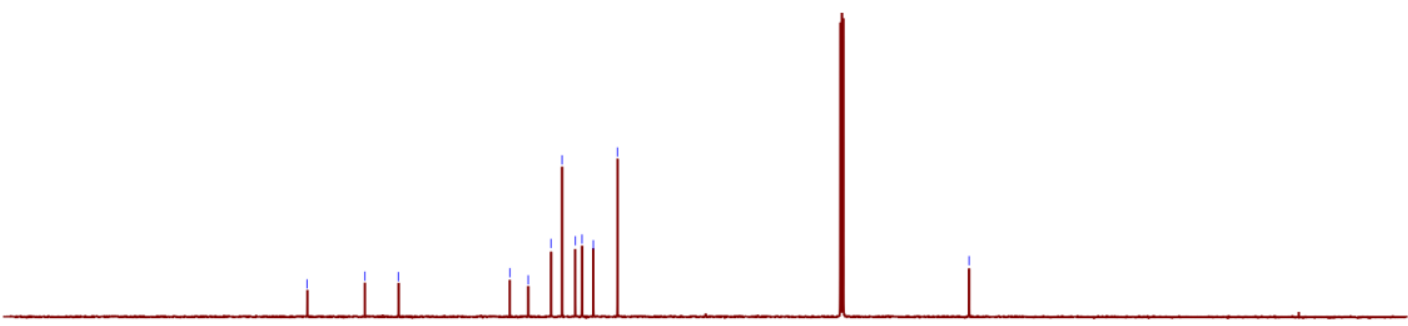

$\begin{array}{llllllllllllllllllllllll}210 & 200 & 190 & 180 & 170 & 160 & 150 & 140 & 130 & 120 & 110 & 100 & 90 & 80 & 70 & 60 & 50 & 40 & 30 & 20 & 10 & 0 & -10\end{array}$ 
${ }^{1} \mathrm{H}$ NMR spectrum for $\mathbf{3 d}$

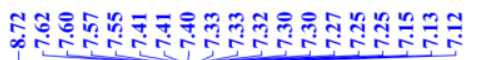

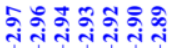

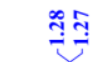

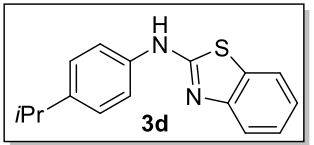

$\mathrm{CDCl}_{3}$

$500 \mathrm{MHz}$

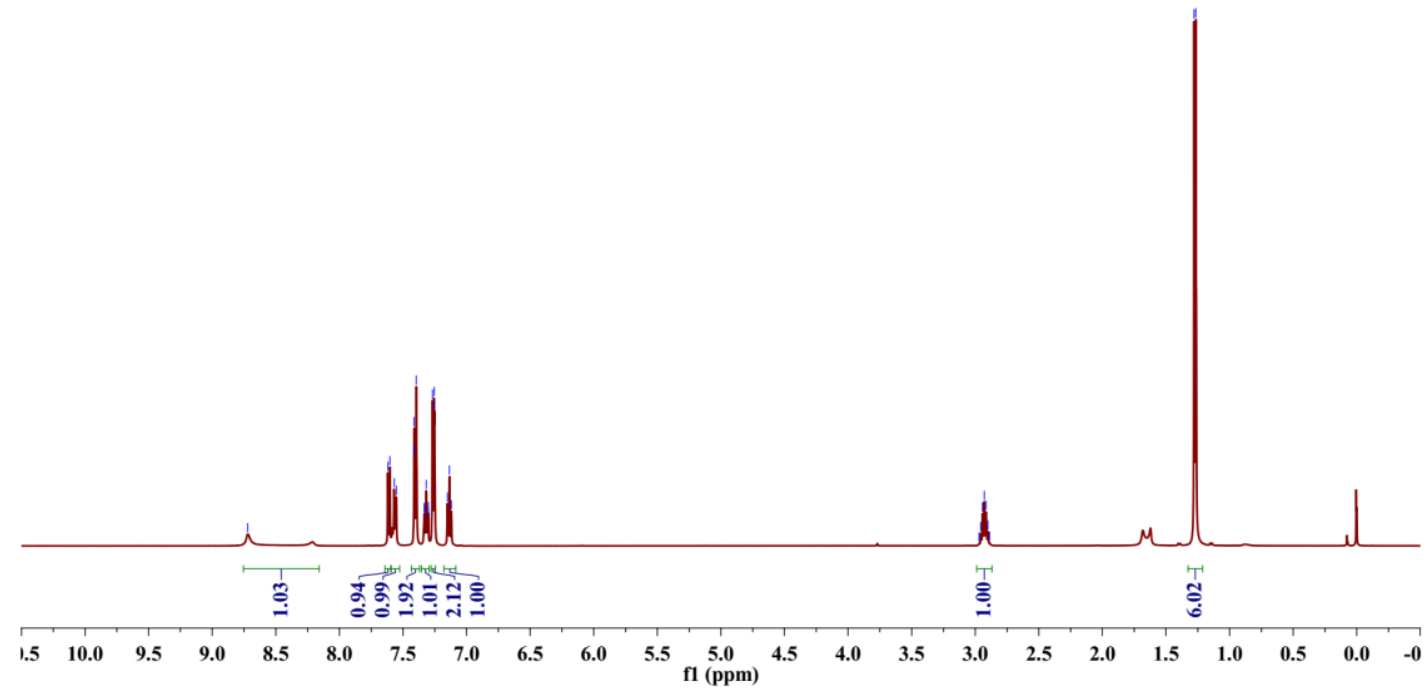

$>{ }^{13} \mathrm{C}$ NMR spectrum for $\mathbf{3 d}$

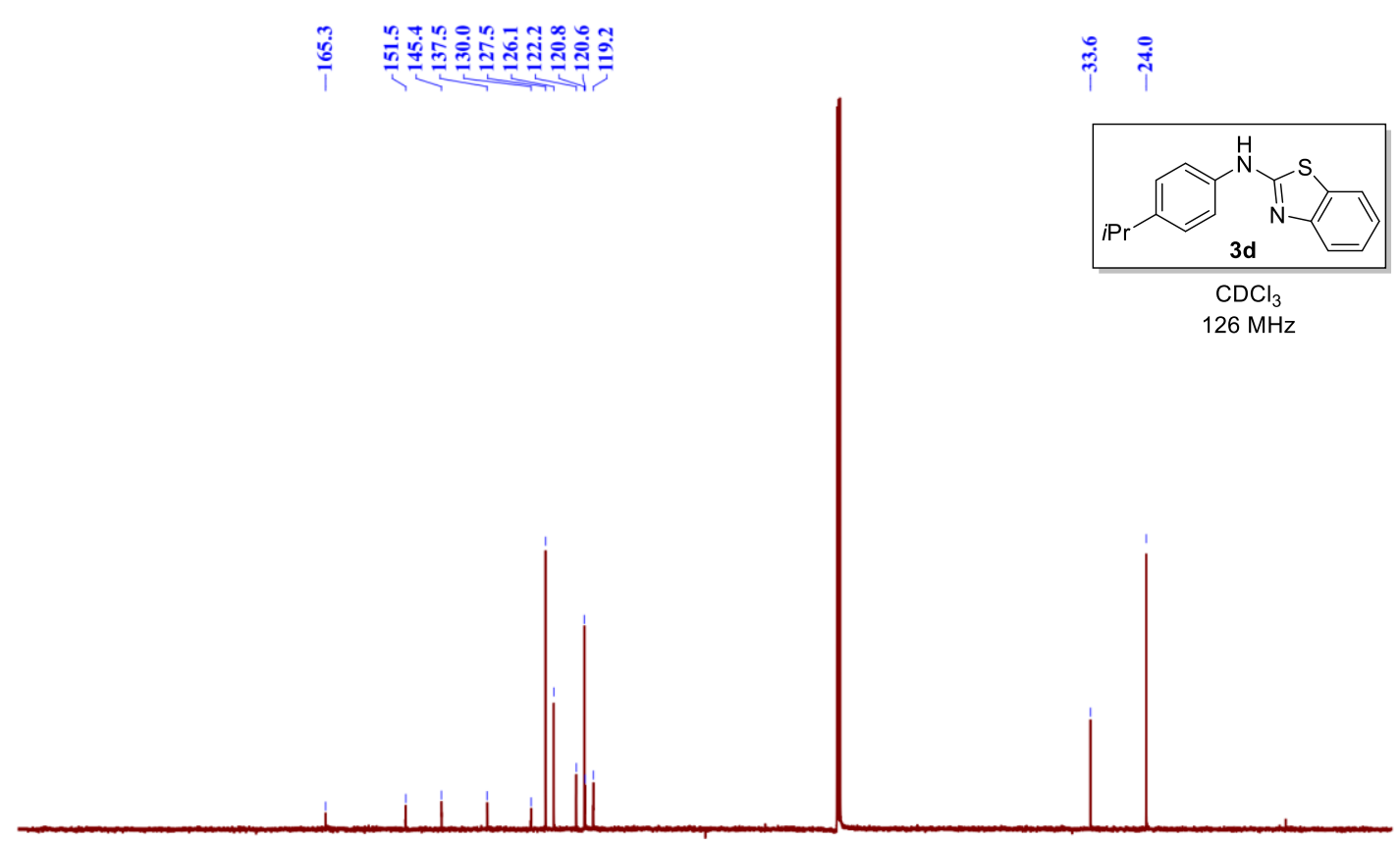

$\begin{array}{llllllllllllllllllllllll}210 & 200 & 190 & 180 & 170 & 160 & 150 & 140 & 130 & 120 & 110 & \begin{array}{l}100 \\ 100\end{array} & 90 & 80 & 70 & 60 & 50 & 40 & 30 & 20 & 10 & 0 & -10 & \end{array}$ 
${ }^{1} \mathrm{H}$ NMR spectrum for $\mathbf{3 e}$

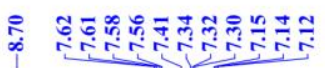

$\frac{\substack{\mathrm{CDCl}_{3} \\ 500 \mathrm{MHz}}}{\overbrace{3 e}^{\mathrm{N}}}$

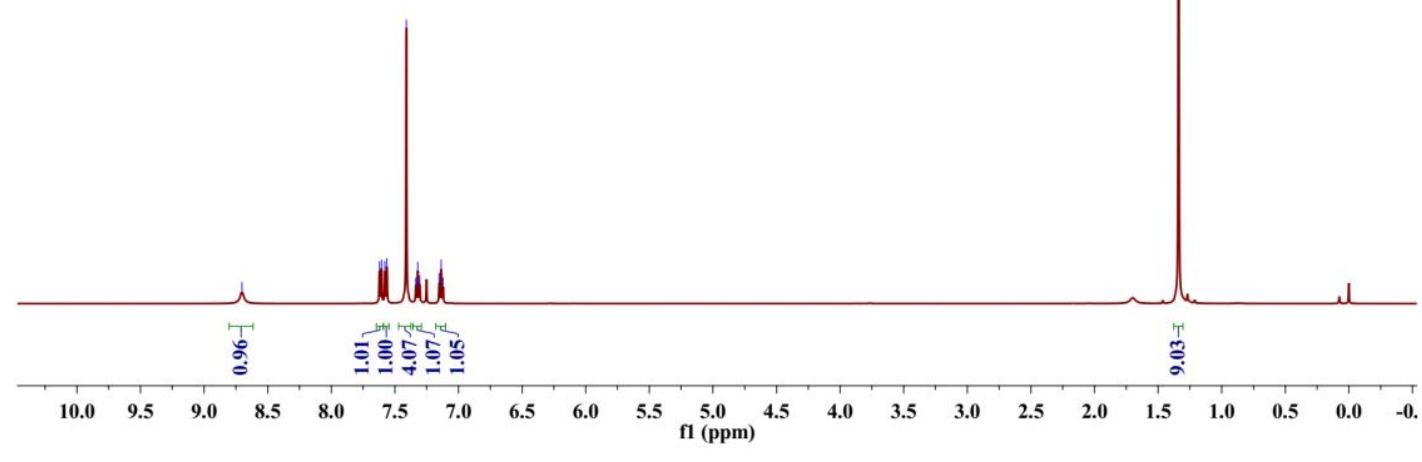

${ }^{13} \mathrm{C}$ NMR spectrum for $\mathbf{3 e}$
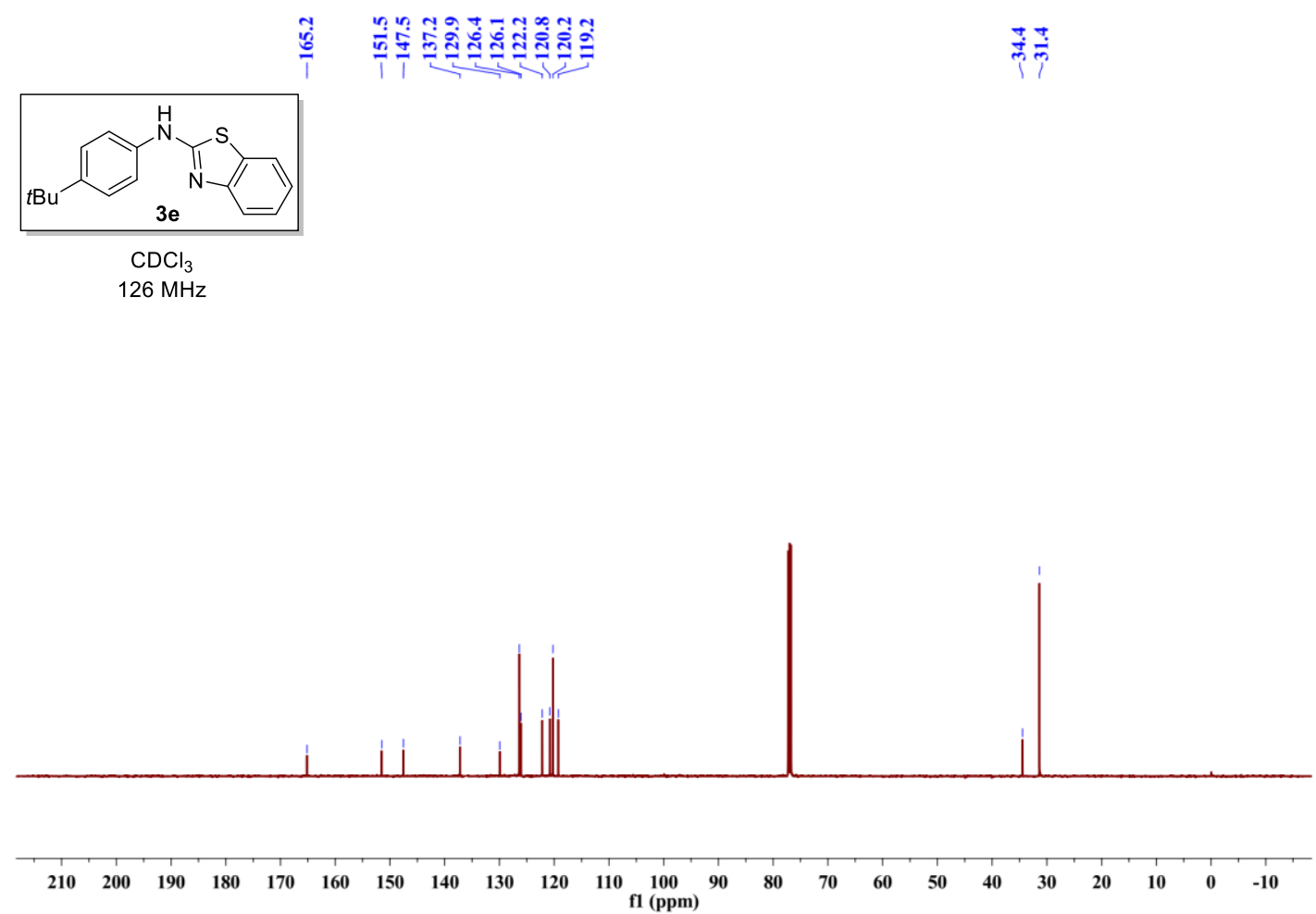
${ }^{1} \mathrm{H}$ NMR spectrum for $\mathbf{3 f}$

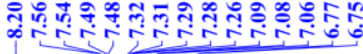

$\stackrel{\infty}{i}$
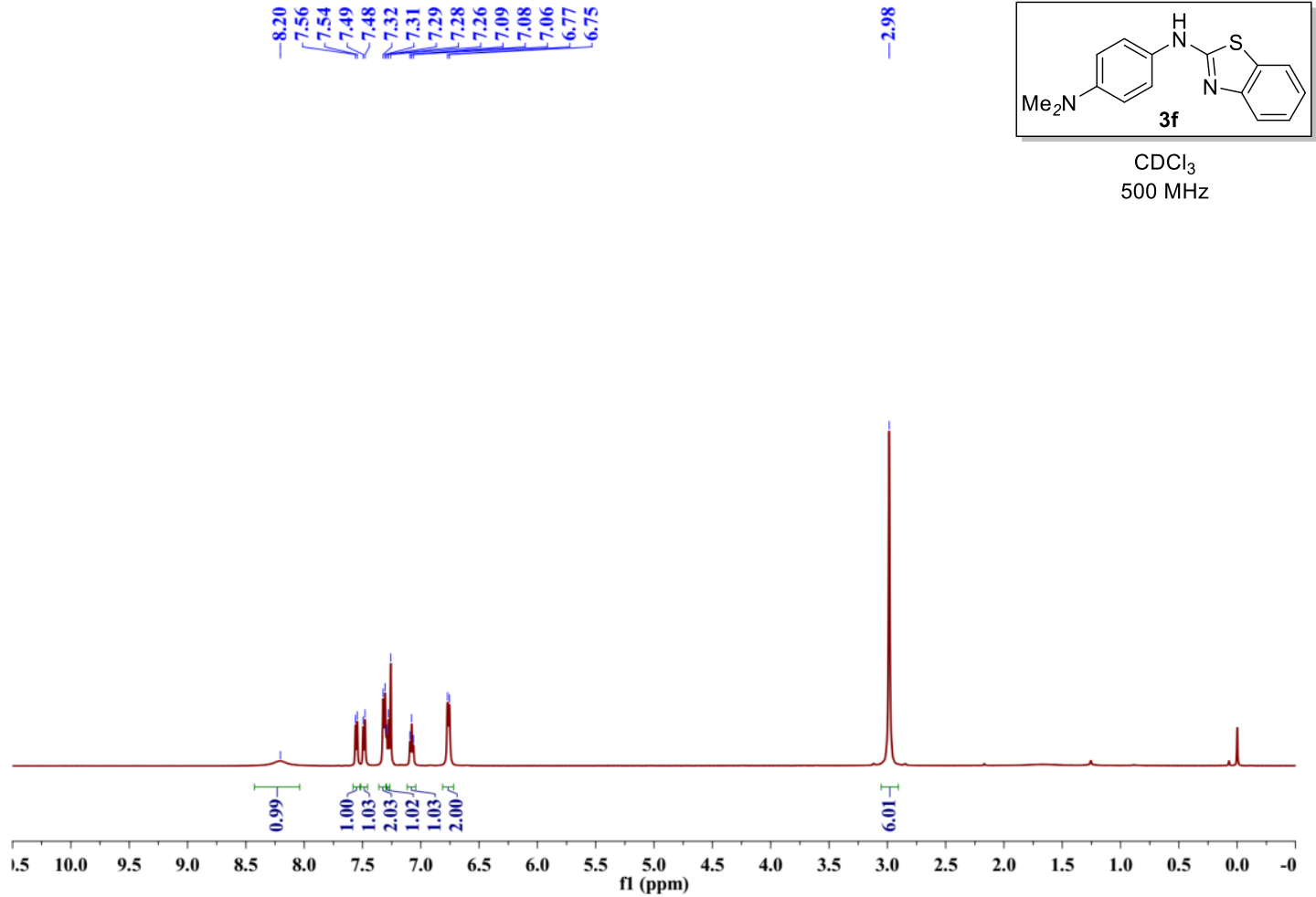

$>{ }^{13} \mathrm{C}$ NMR spectrum for $\mathbf{3 f}$

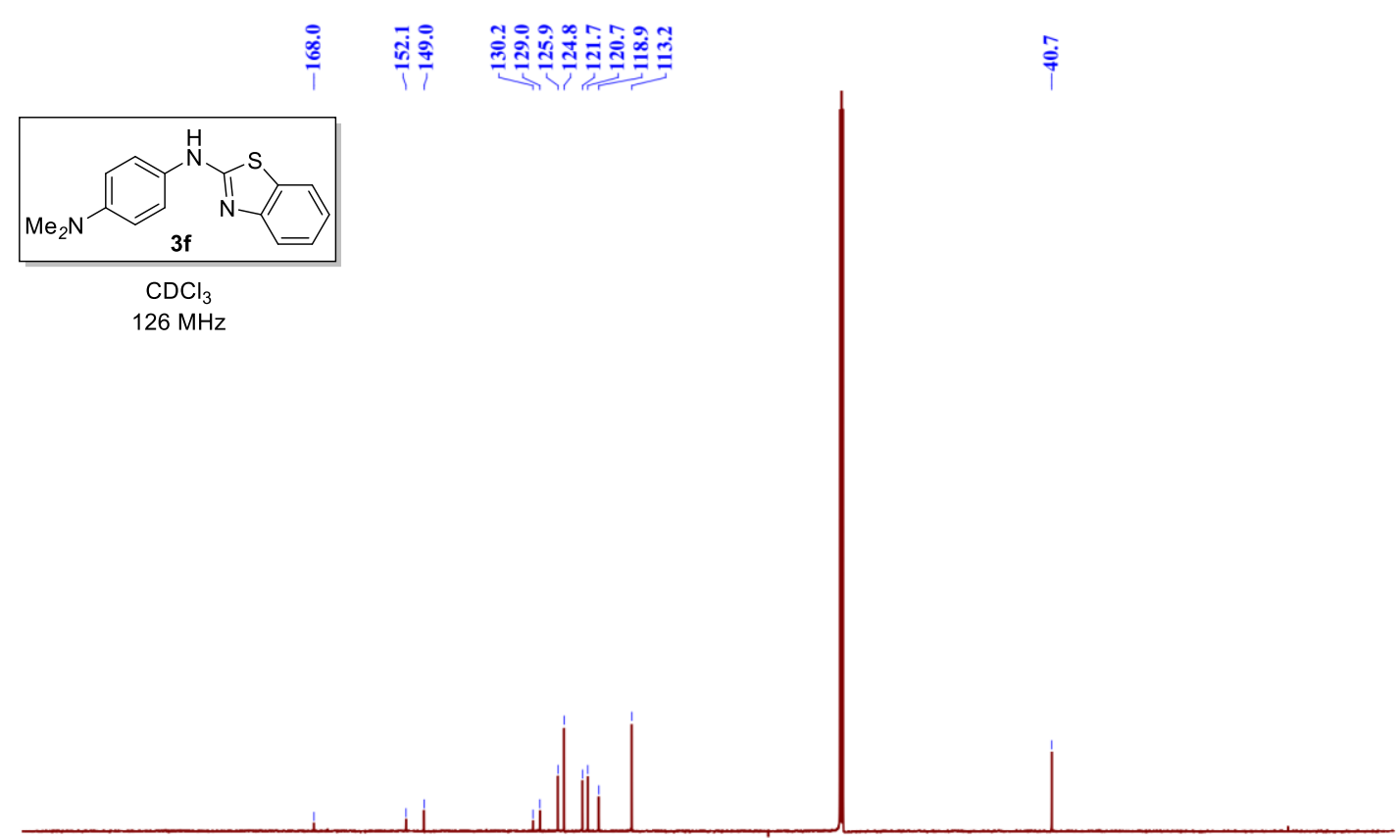

$\begin{array}{llllllllllllllllllllllll}210 & 200 & 190 & 180 & 170 & 160 & 150 & 140 & 130 & 120 & 110 & \begin{array}{c}100 \\ \mathrm{fl}(\mathrm{ppm})\end{array} & 90 & 80 & 70 & 60 & 50 & 40 & 30 & 20 & 10 & 0 & -10 & \end{array}$ 
${ }^{1} \mathrm{H}$ NMR spectrum for $\mathbf{3 g}$

要
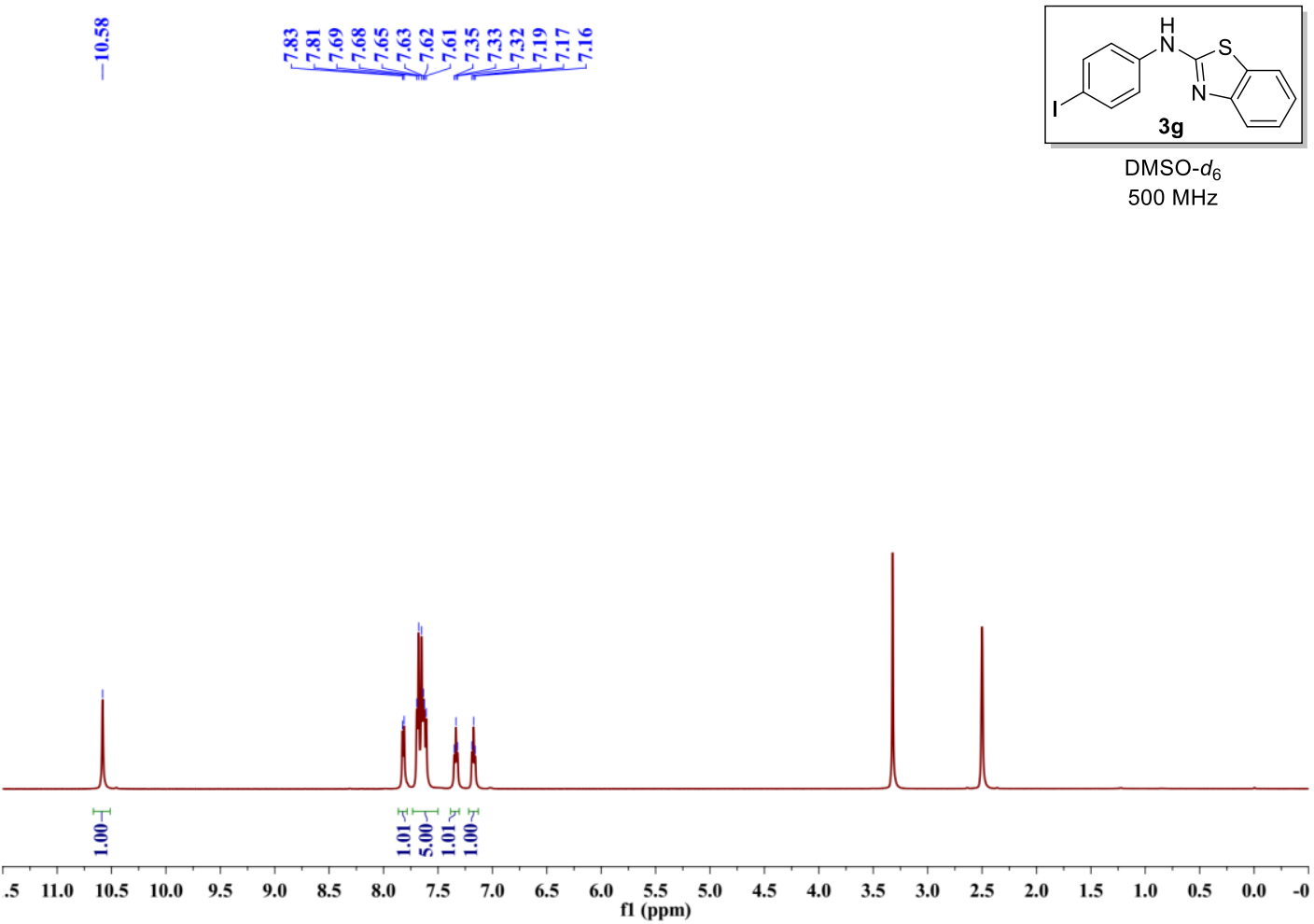

$>{ }^{13} \mathrm{C}$ NMR spectrum for $\mathbf{3 g}$

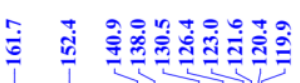

$\stackrel{3}{0}$
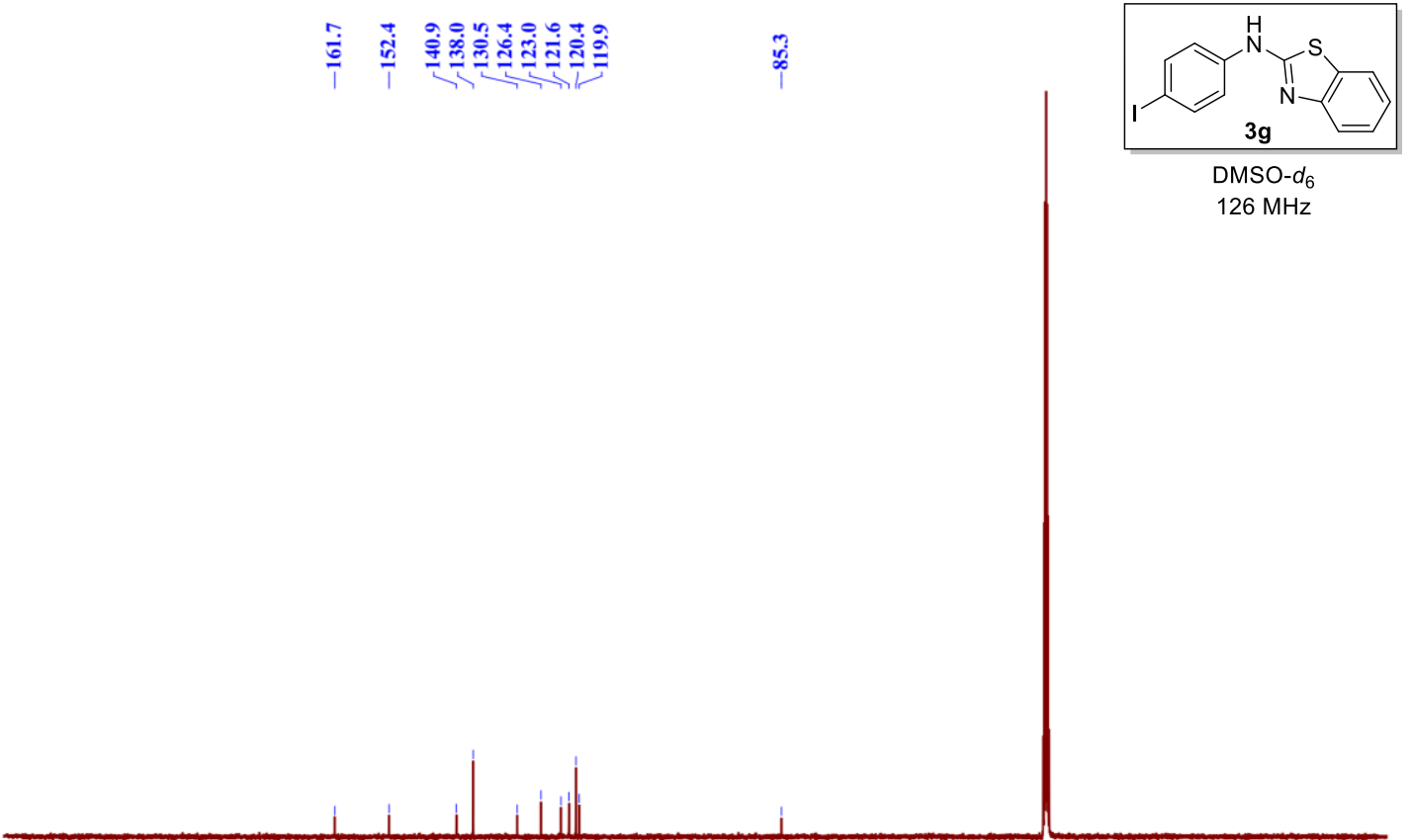

$\begin{array}{llllllllllllllllllllllll}210 & 200 & 190 & 180 & 170 & 160 & 150 & 140 & 130 & 120 & 110 & 100 & 90 & 80 & 70 & 60 & 50 & 40 & 30 & 20 & 10 & 0 & -10 & \end{array}$ 
${ }^{1} \mathrm{H}$ NMR spectrum for $\mathbf{3 h}$

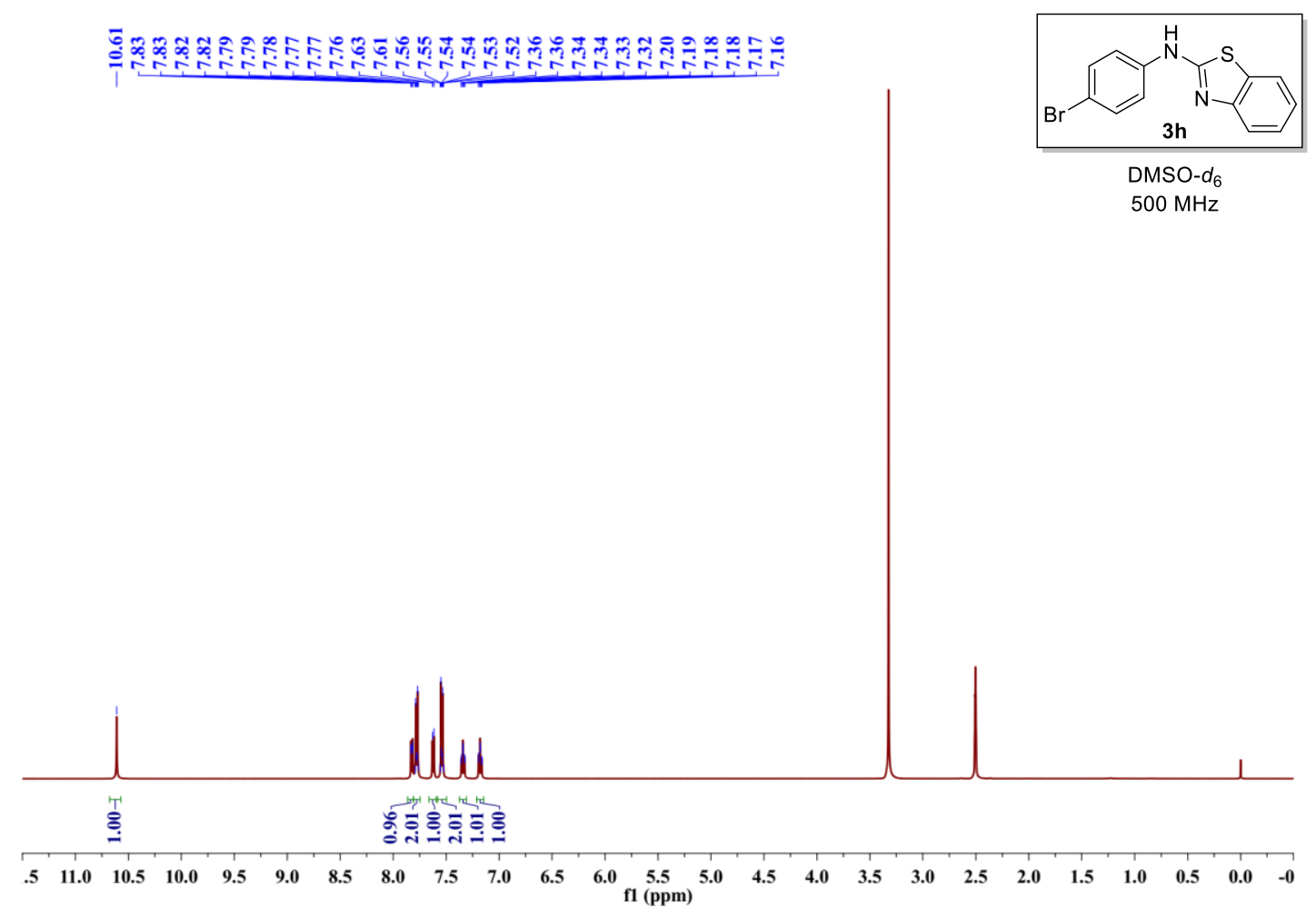

$>{ }^{13} \mathrm{C}$ NMR spectrum for $\mathbf{3 h}$

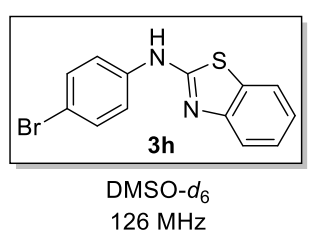

†

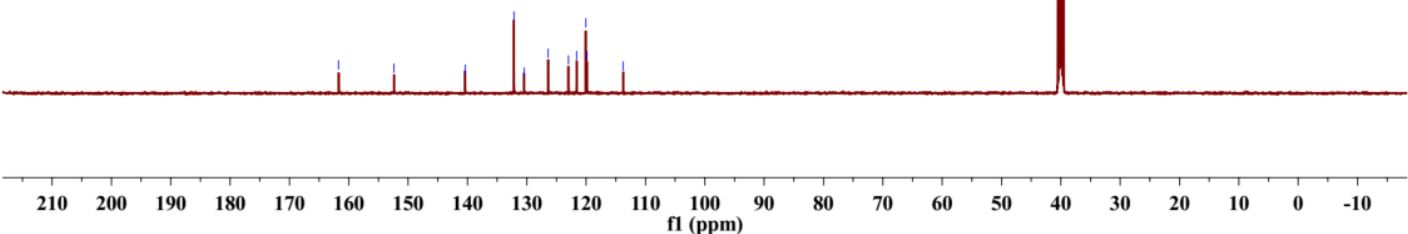


${ }^{1} \mathrm{H}$ NMR spectrum for $\mathbf{3 i}$

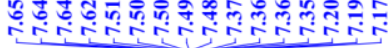
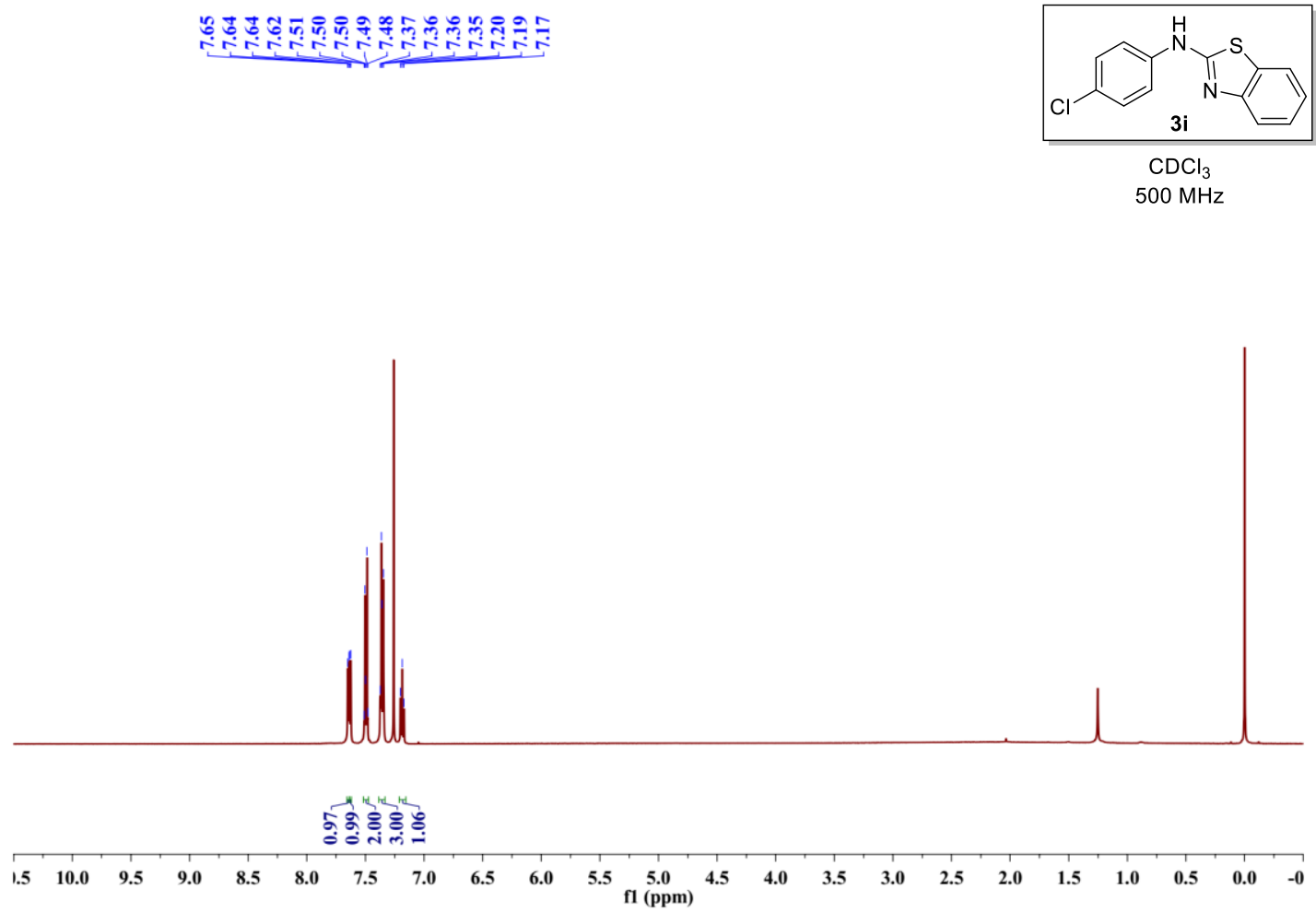

$>{ }^{13} \mathrm{C}$ NMR spectrum for $\mathbf{3 i}$

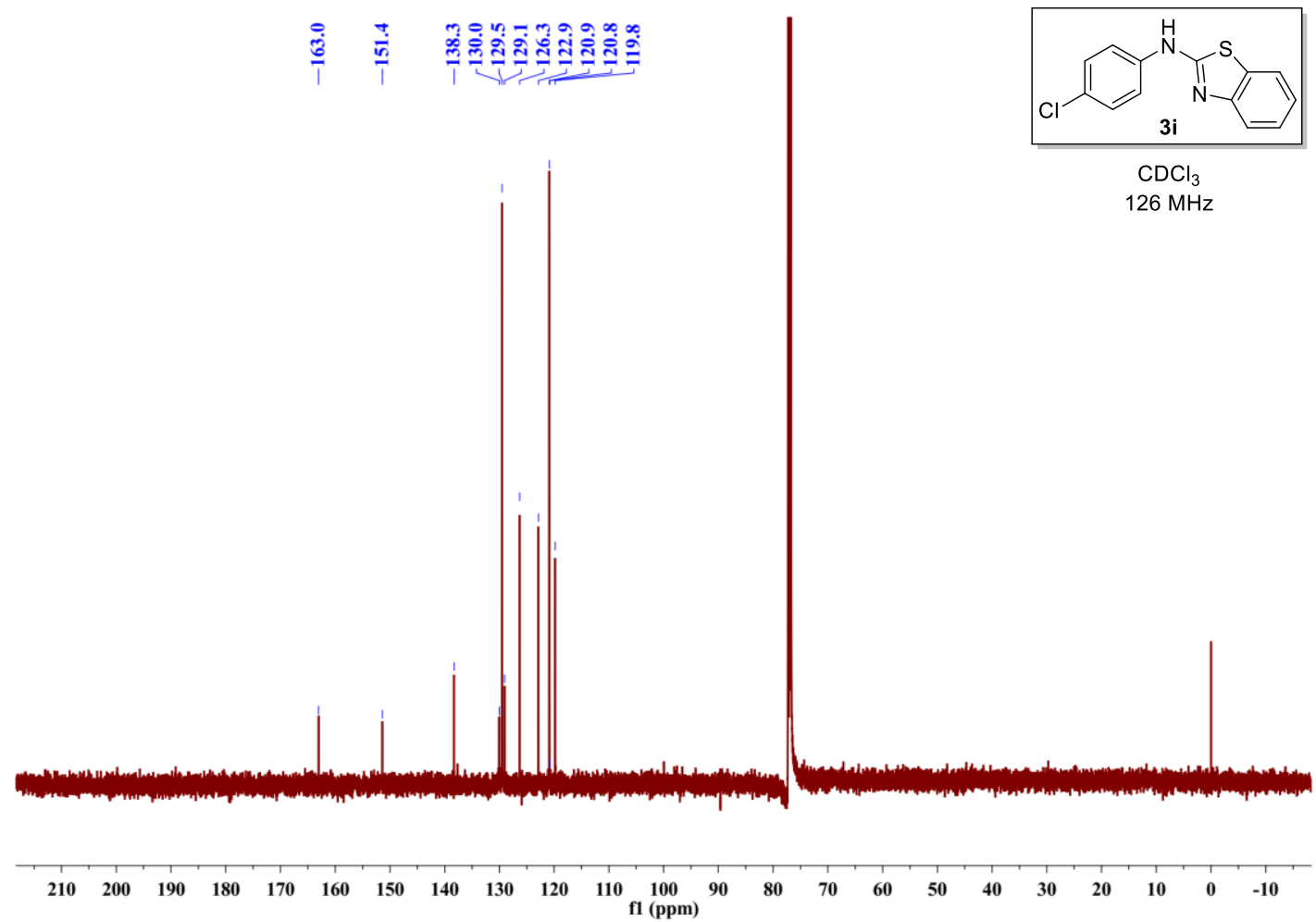


${ }^{1} \mathrm{H}$ NMR spectrum for $\mathbf{3 j}$
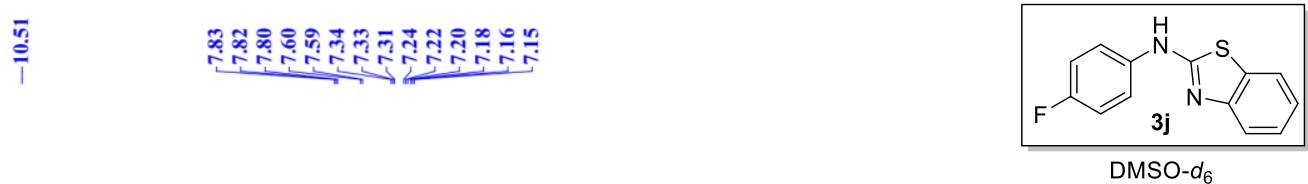

$500 \mathrm{MHz}$

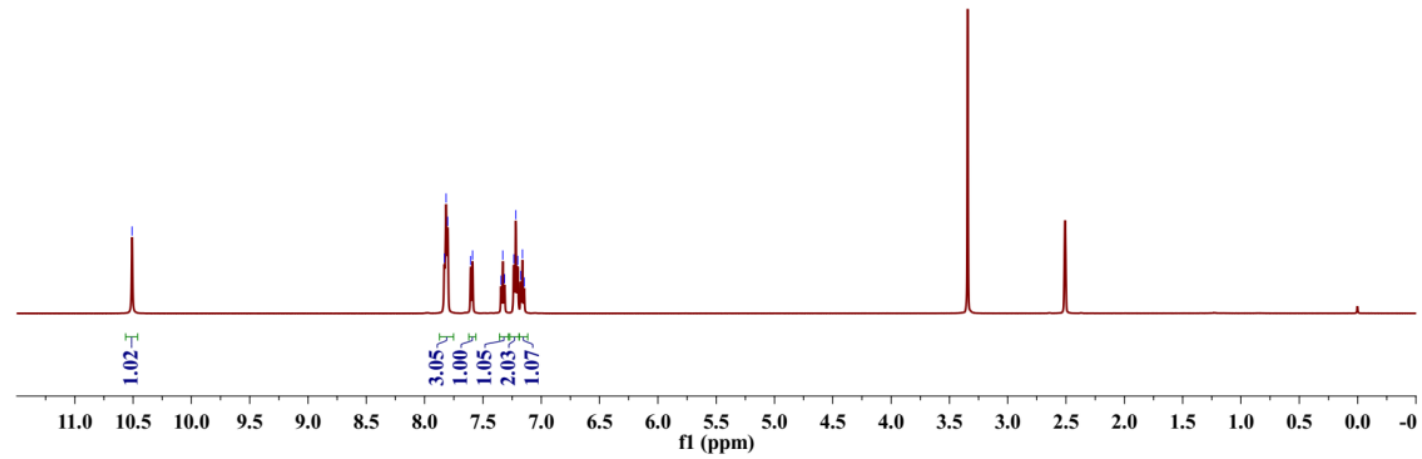

$>{ }^{13} \mathrm{C}$ NMR spectrum for $\mathbf{3 j}$

7ن.

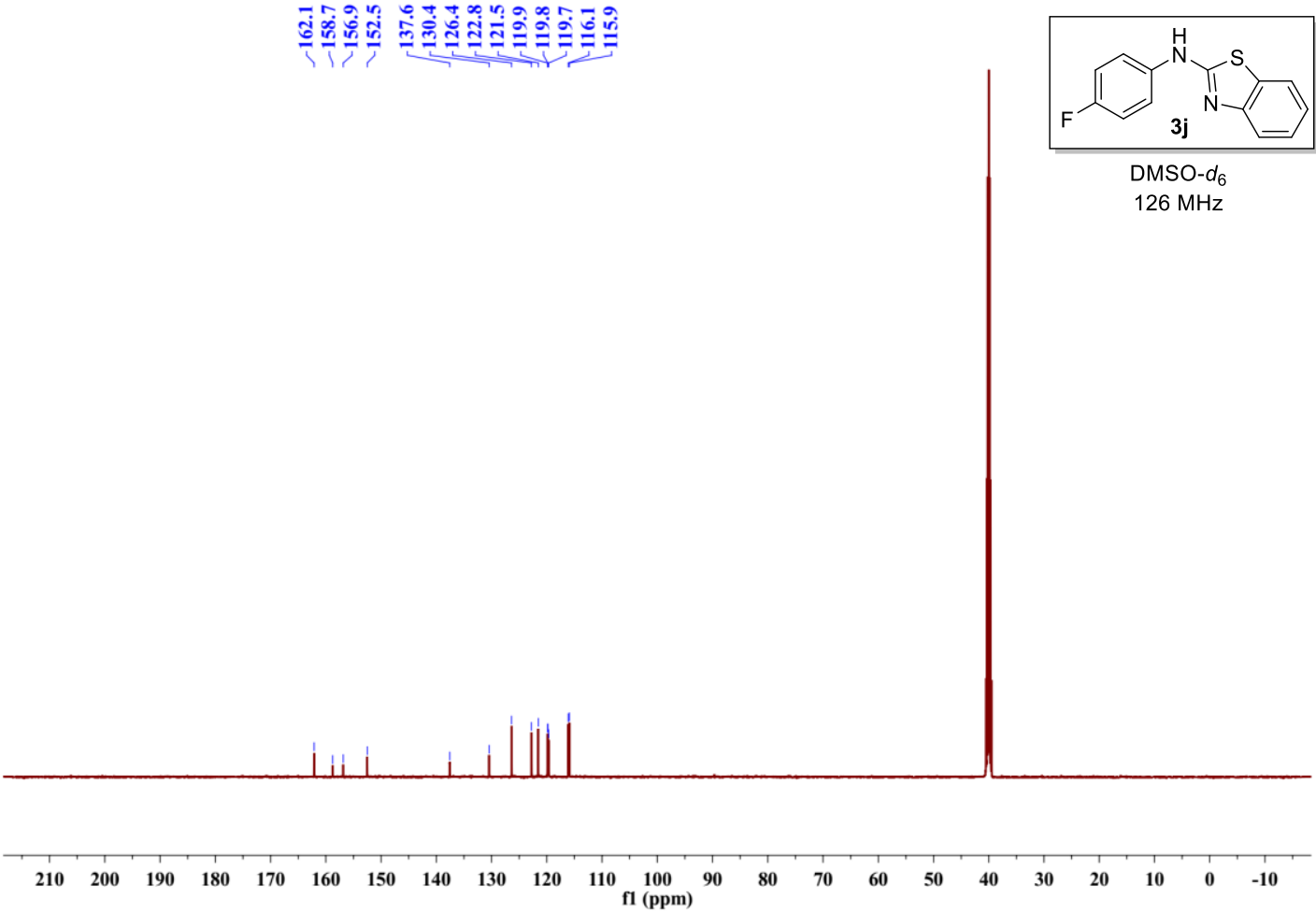


${ }^{19}$ F NMR spectrum for $\mathbf{3 j}$

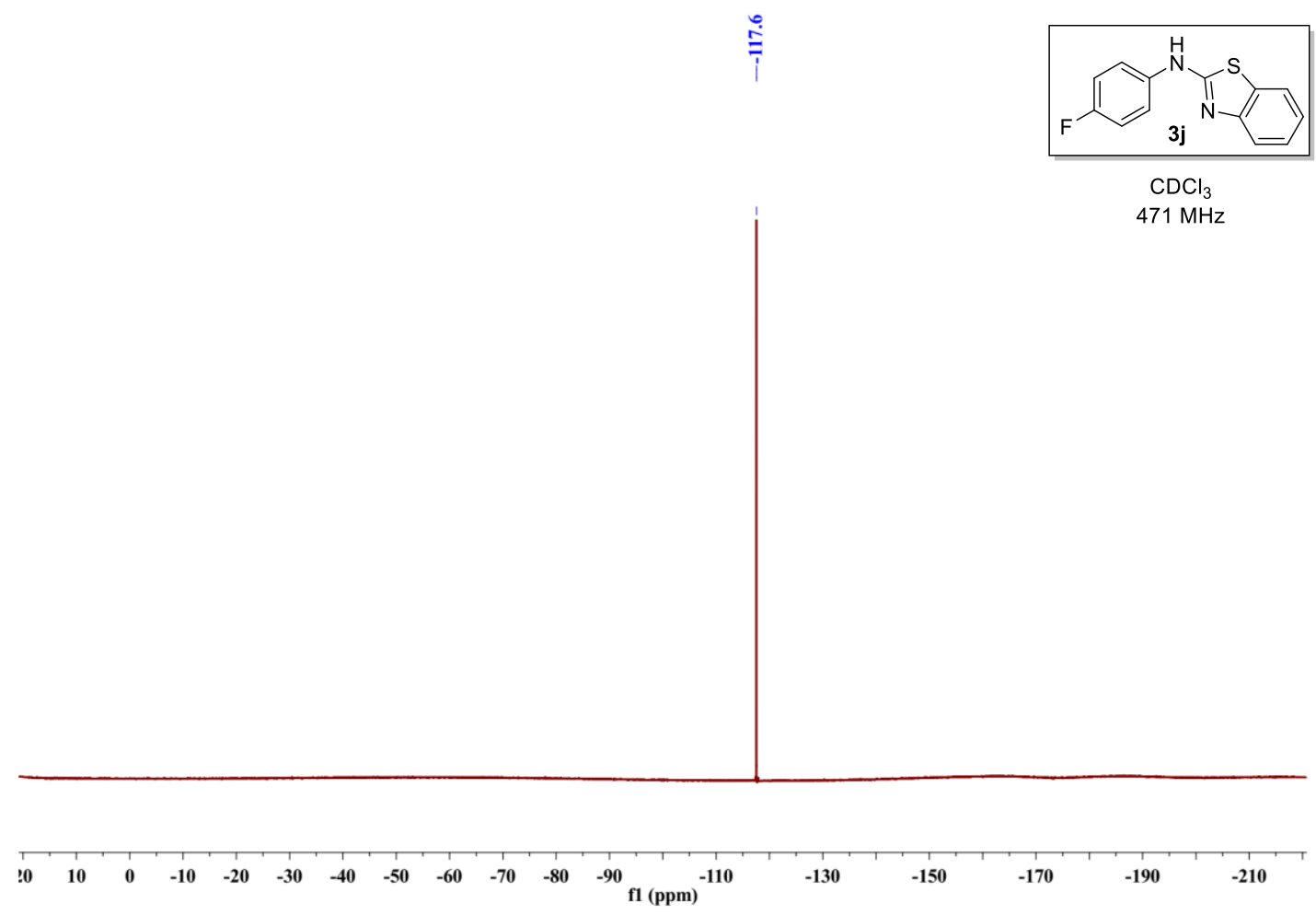


${ }^{1} \mathrm{H}$ NMR spectrum for $\mathbf{3 k}$

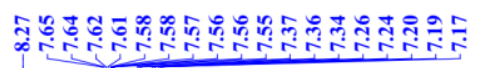
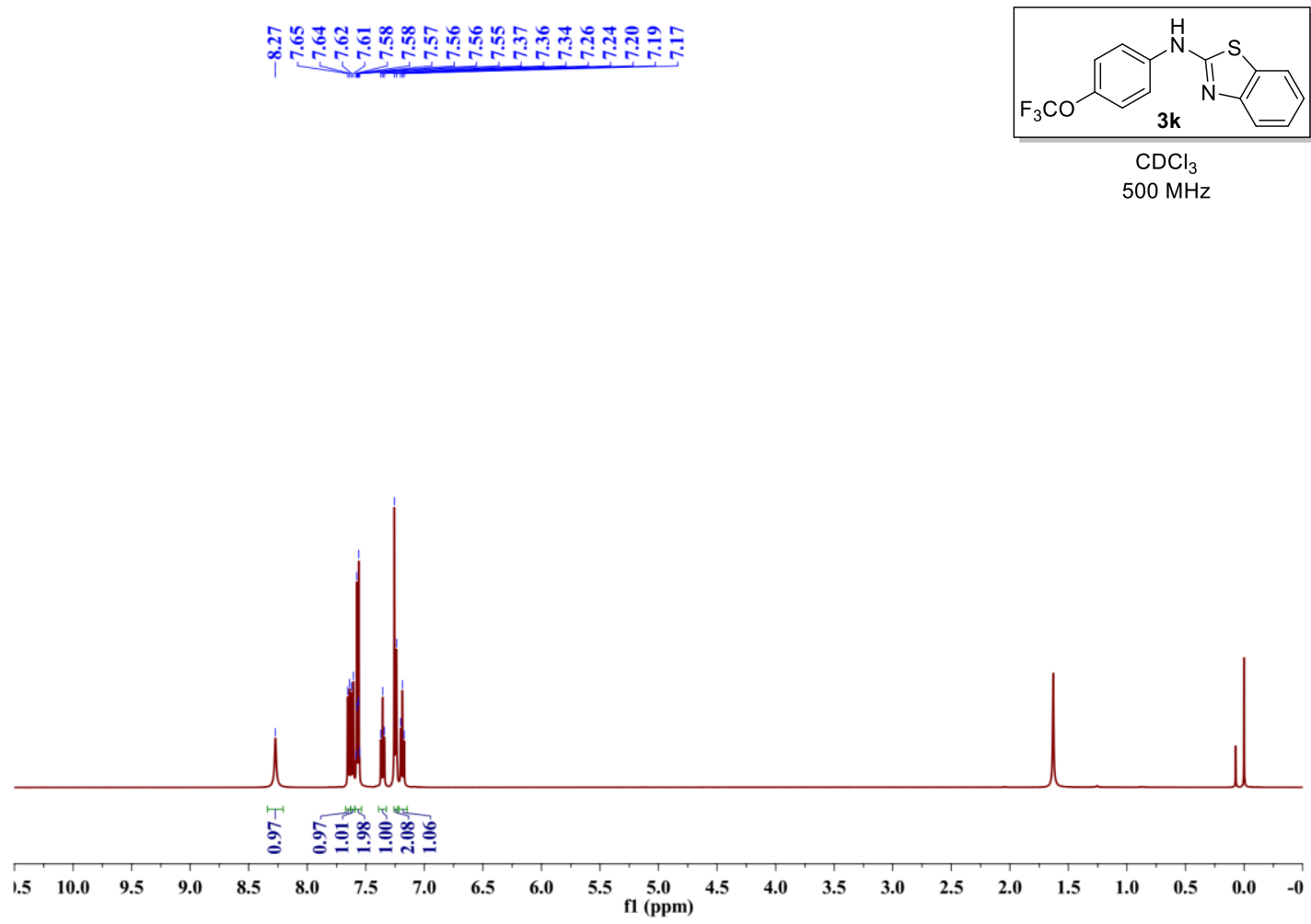

$>{ }^{13} \mathrm{C}$ NMR spectrum for $\mathbf{3 k}$

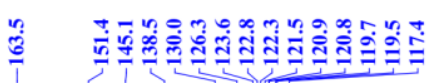

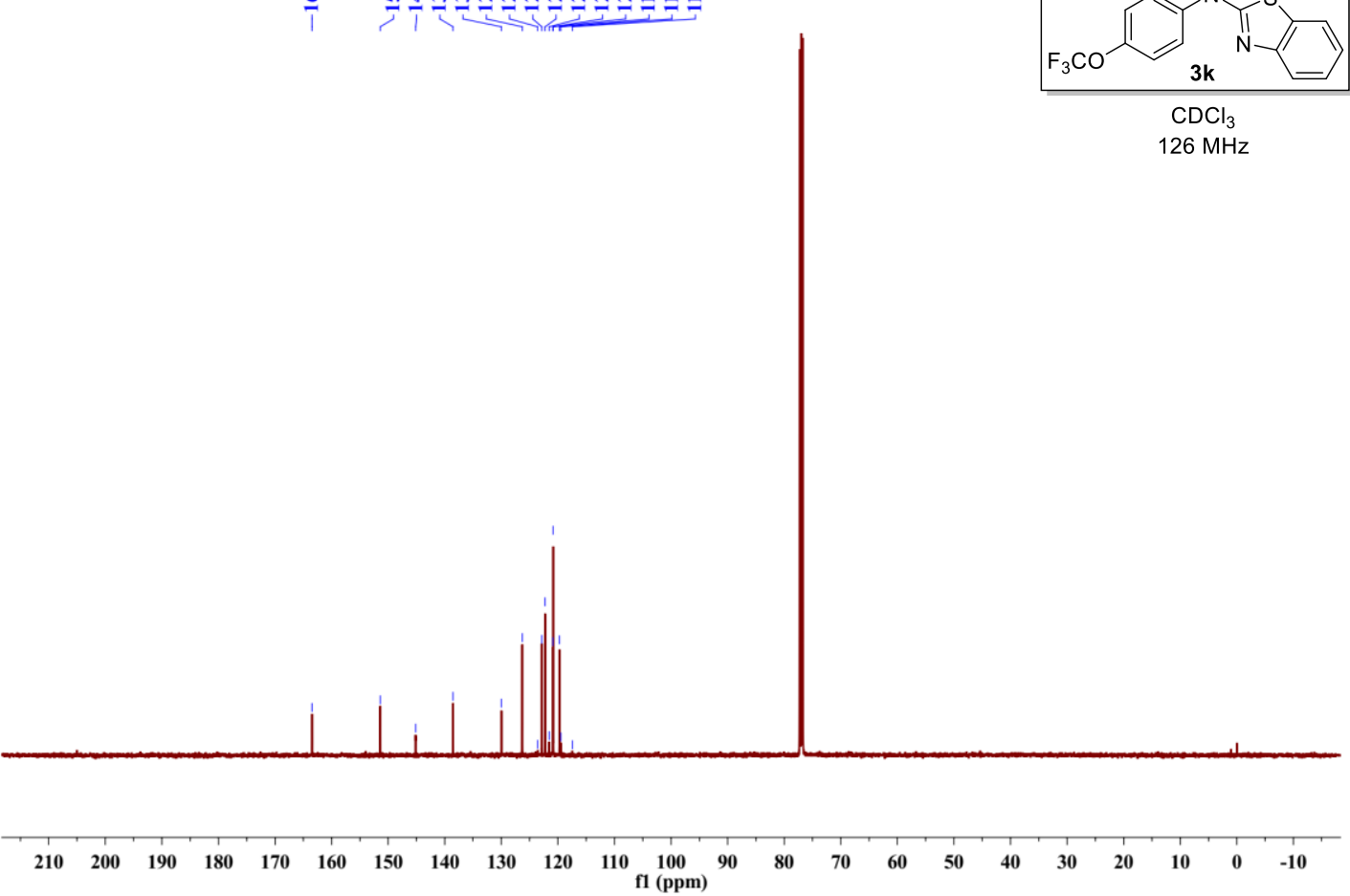


${ }^{19} \mathrm{~F}$ NMR spectrum for $\mathbf{3 k}$

iุ

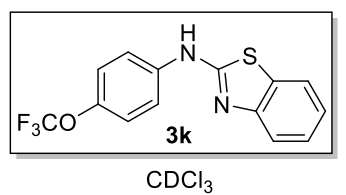

$471 \mathrm{MHz}$

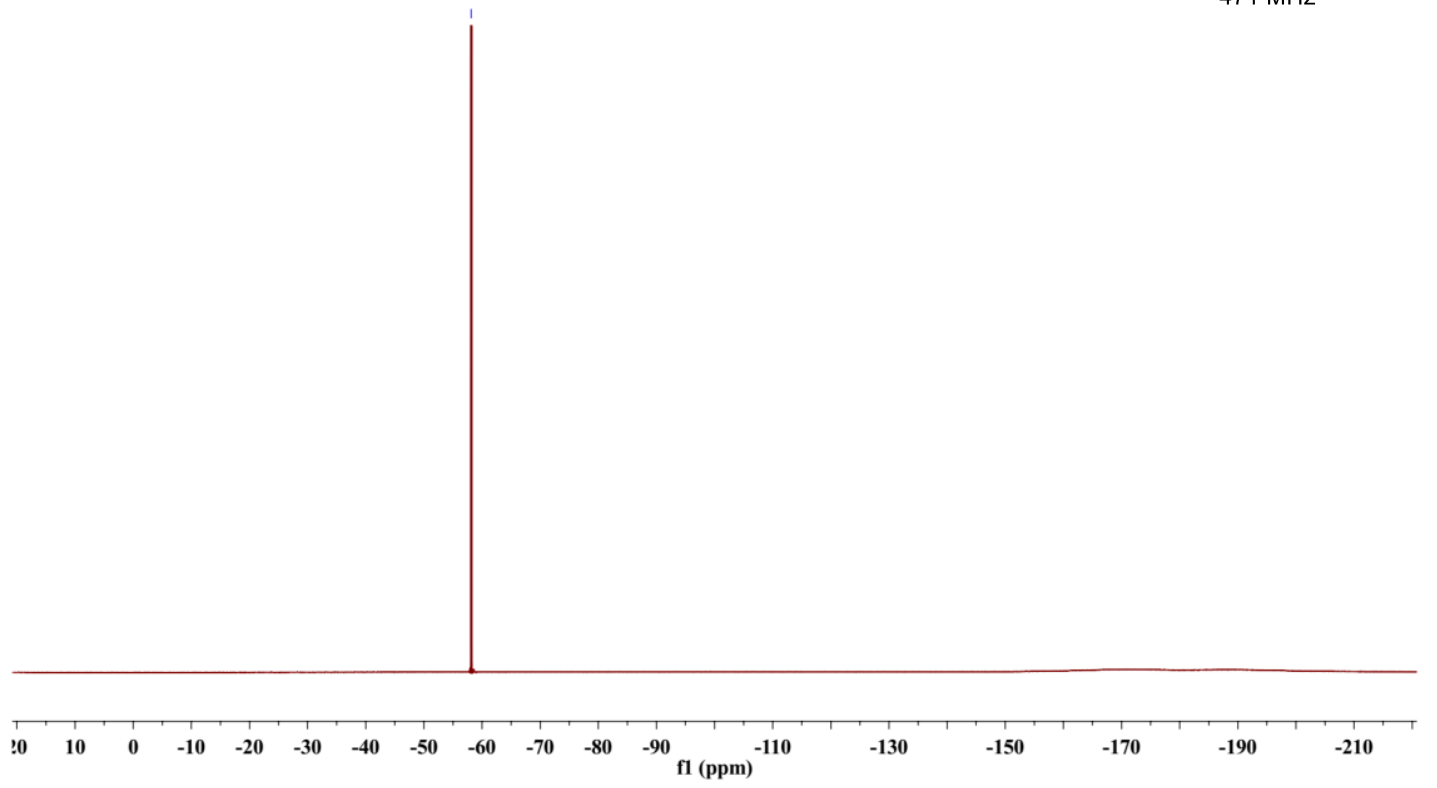


$>{ }^{1} \mathrm{H}$ NMR spectrum for $\mathbf{3 l}$

il

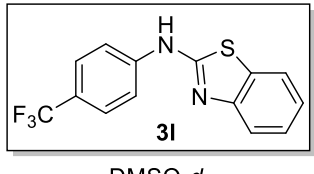

DMSO- $d_{6}$

$500 \mathrm{MHz}$

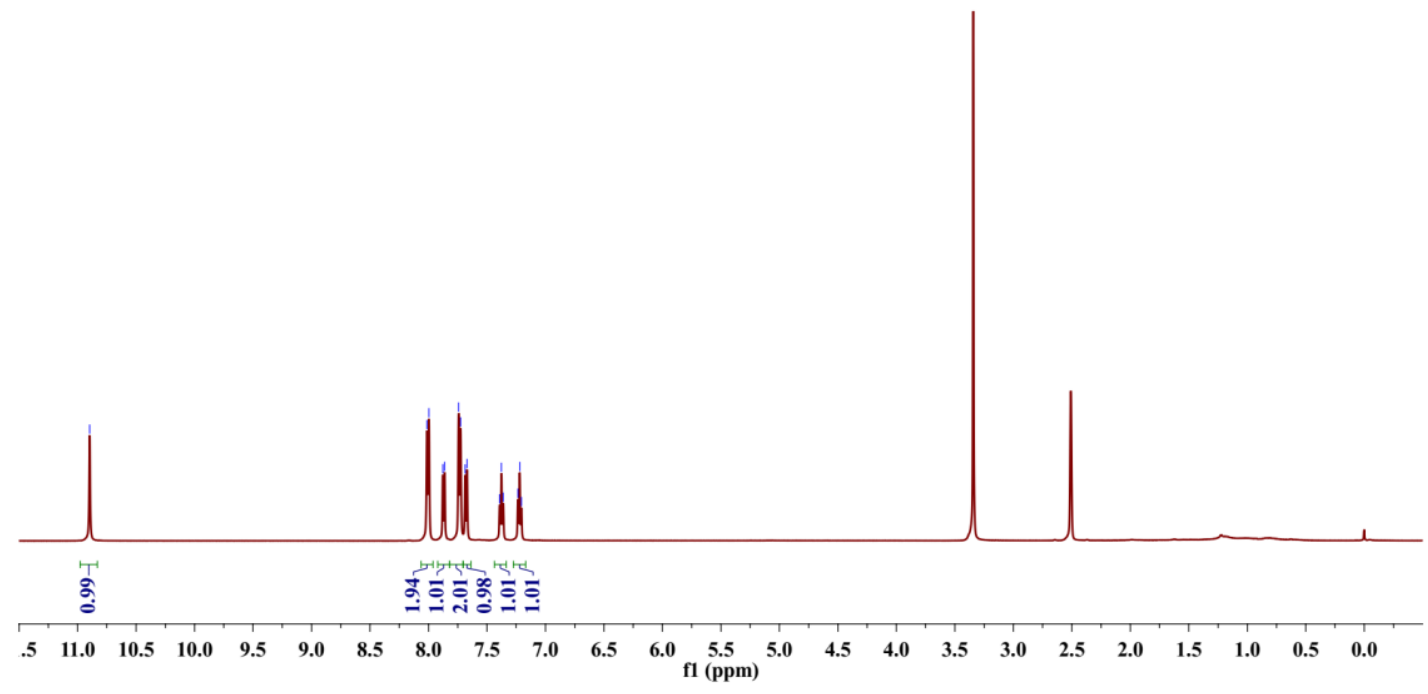

$>{ }^{13} \mathrm{C}$ NMR spectrum for $\mathbf{3 I}$

应

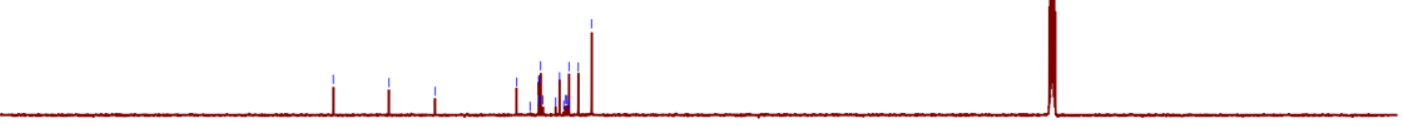

$\begin{array}{lllllllllllllllllllllll}210 & 200 & 190 & 180 & 170 & 160 & 150 & 140 & 130 & 120 & 110 & 100 & 90 & 80 & 70 & 60 & 50 & 40 & 30 & 20 & 10 & 0 & -10\end{array}$ 
${ }^{19} \mathrm{~F}$ NMR spectrum for $\mathbf{3 I}$

$\stackrel{9}{\grave{i}}$
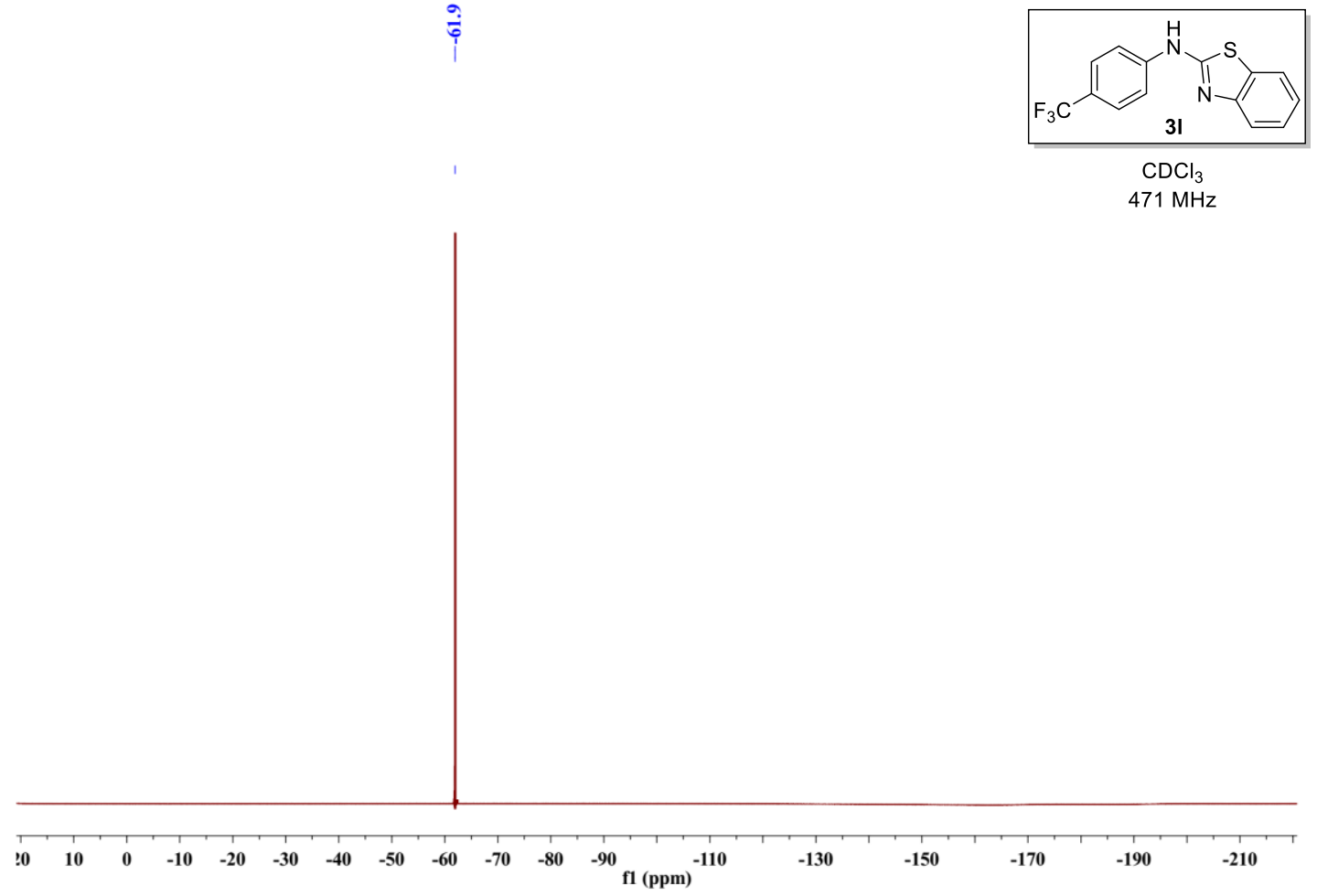
$>{ }^{1} \mathrm{H}$ NMR spectrum for $\mathbf{3 m}$

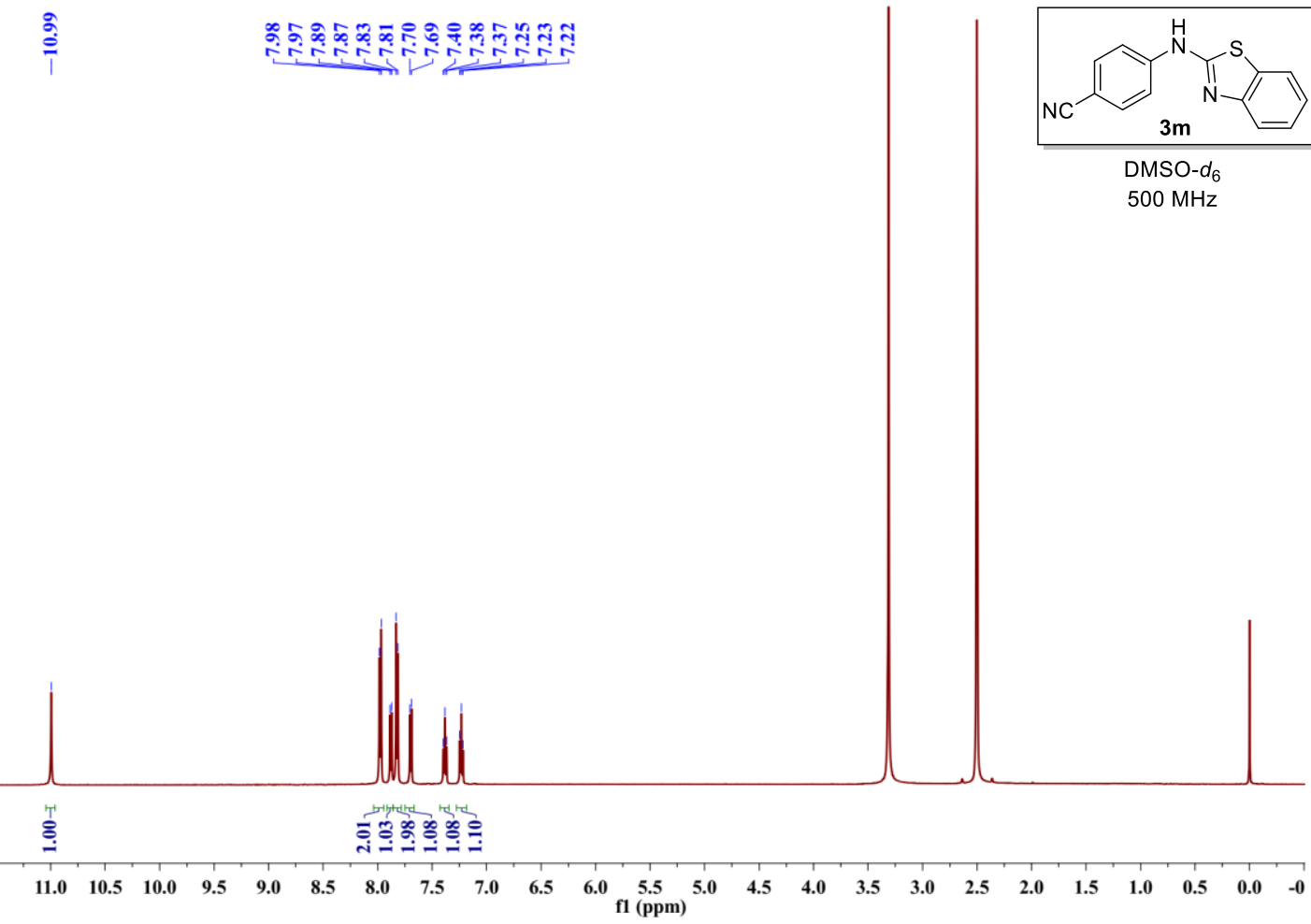

$>{ }^{13} \mathrm{C}$ NMR spectrum for $\mathbf{3 m}$

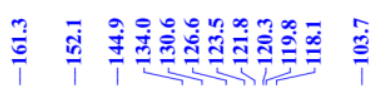

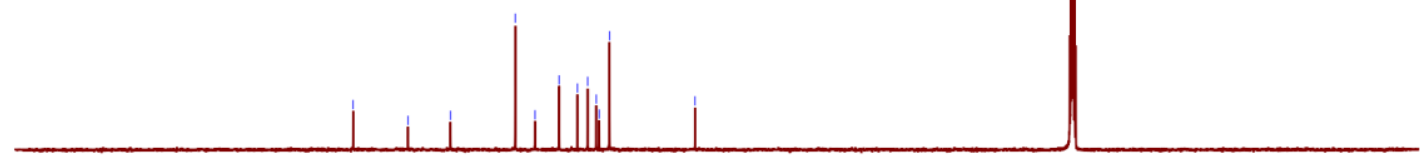

$\begin{array}{lllllllllllllllllllllllll}210 & 200 & 190 & 180 & 170 & 160 & 150 & 140 & 130 & 120 & 110 & 100 & 90 & 80 & 70 & 60 & 50 & 40 & 30 & 20 & 10 & 0 & -10\end{array}$ 
${ }^{1} \mathrm{H}$ NMR spectrum for $\mathbf{3 n}$

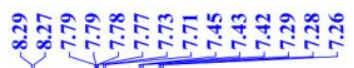
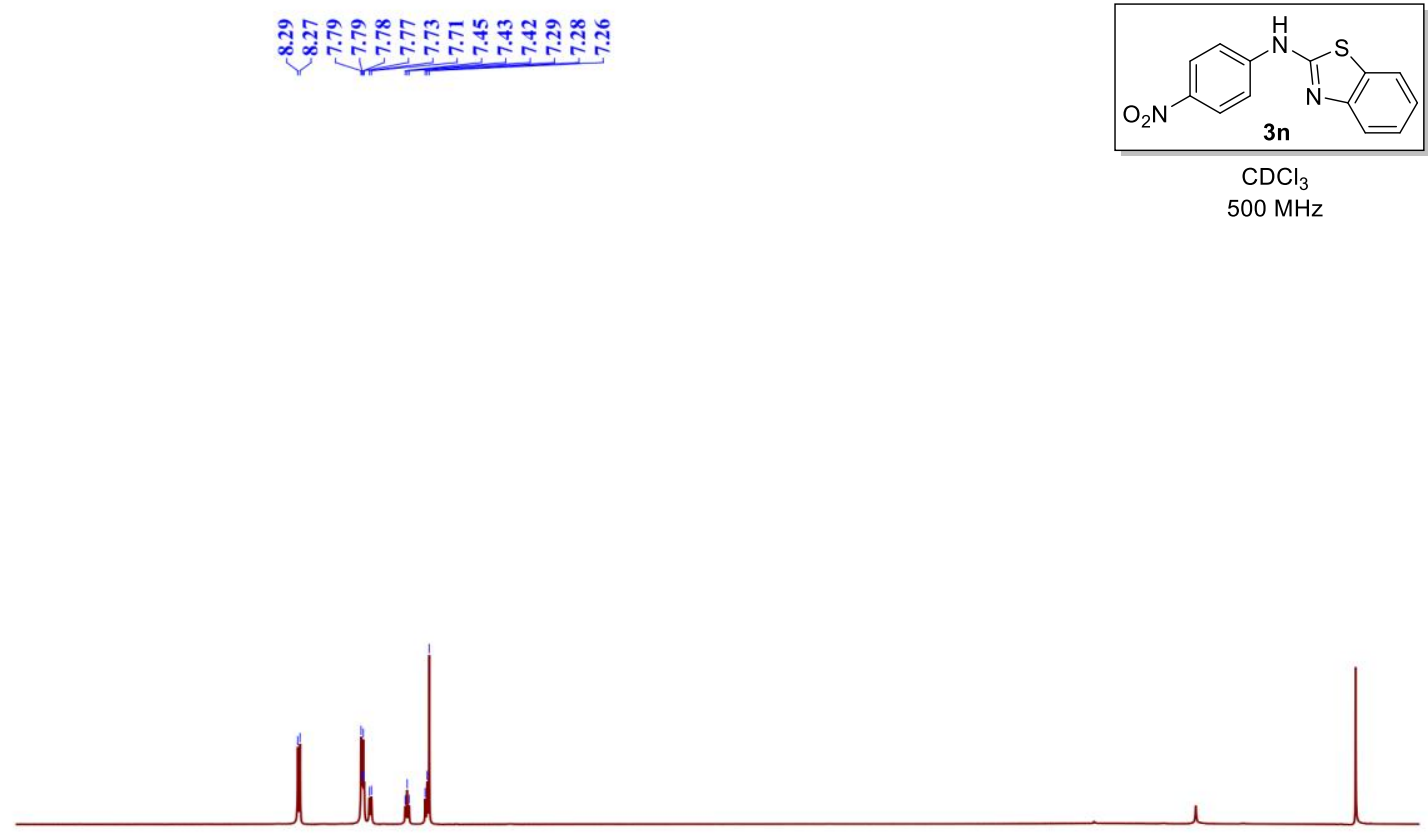

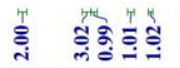

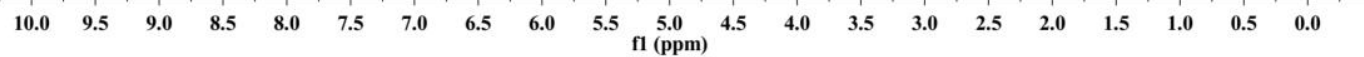

$>{ }^{13} \mathrm{C}$ NMR spectrum for $3 \mathbf{n}$

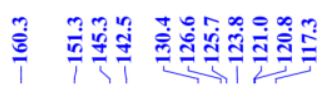
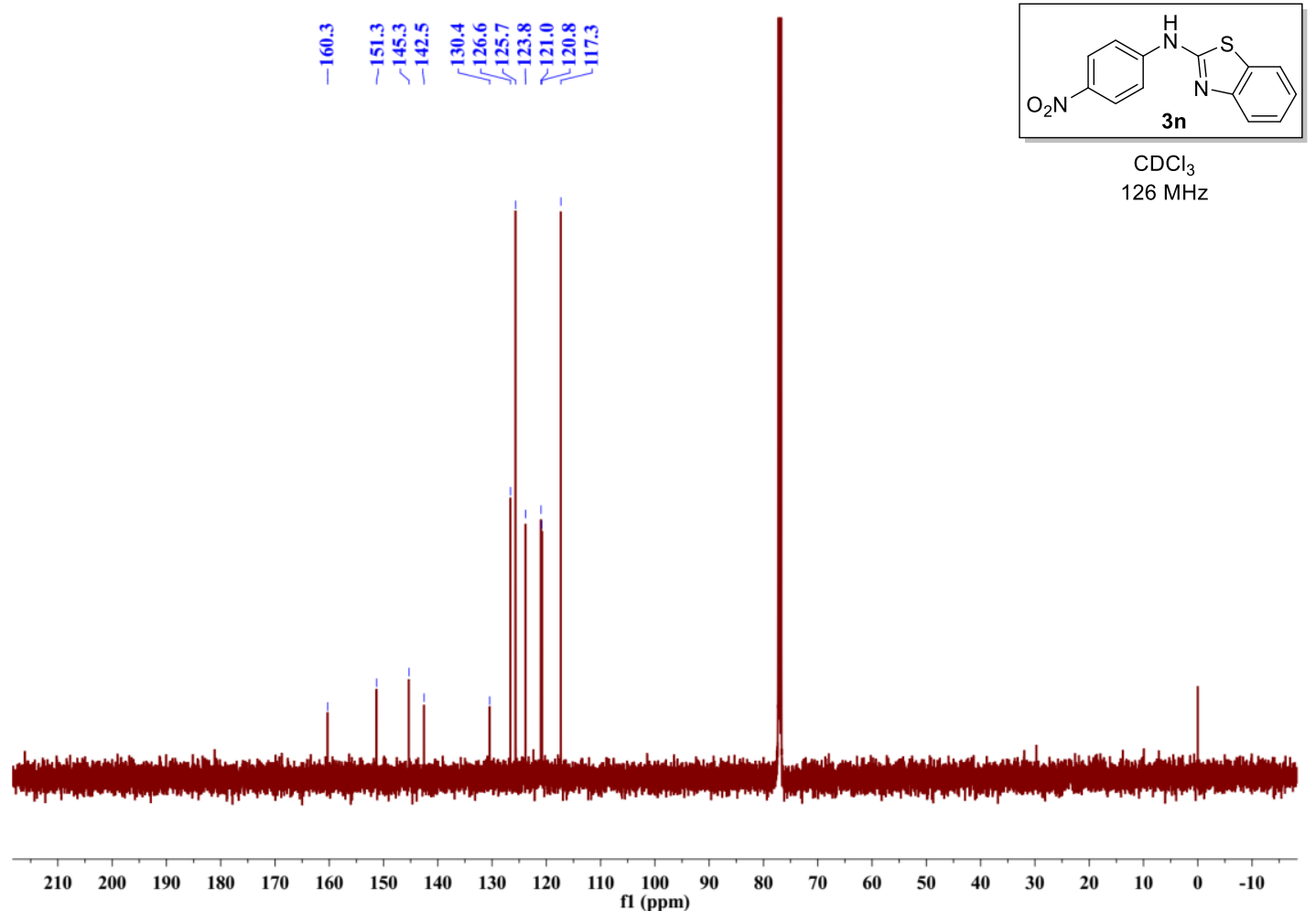
${ }^{1} \mathrm{H}$ NMR spectrum for $\mathbf{3 o}$

5
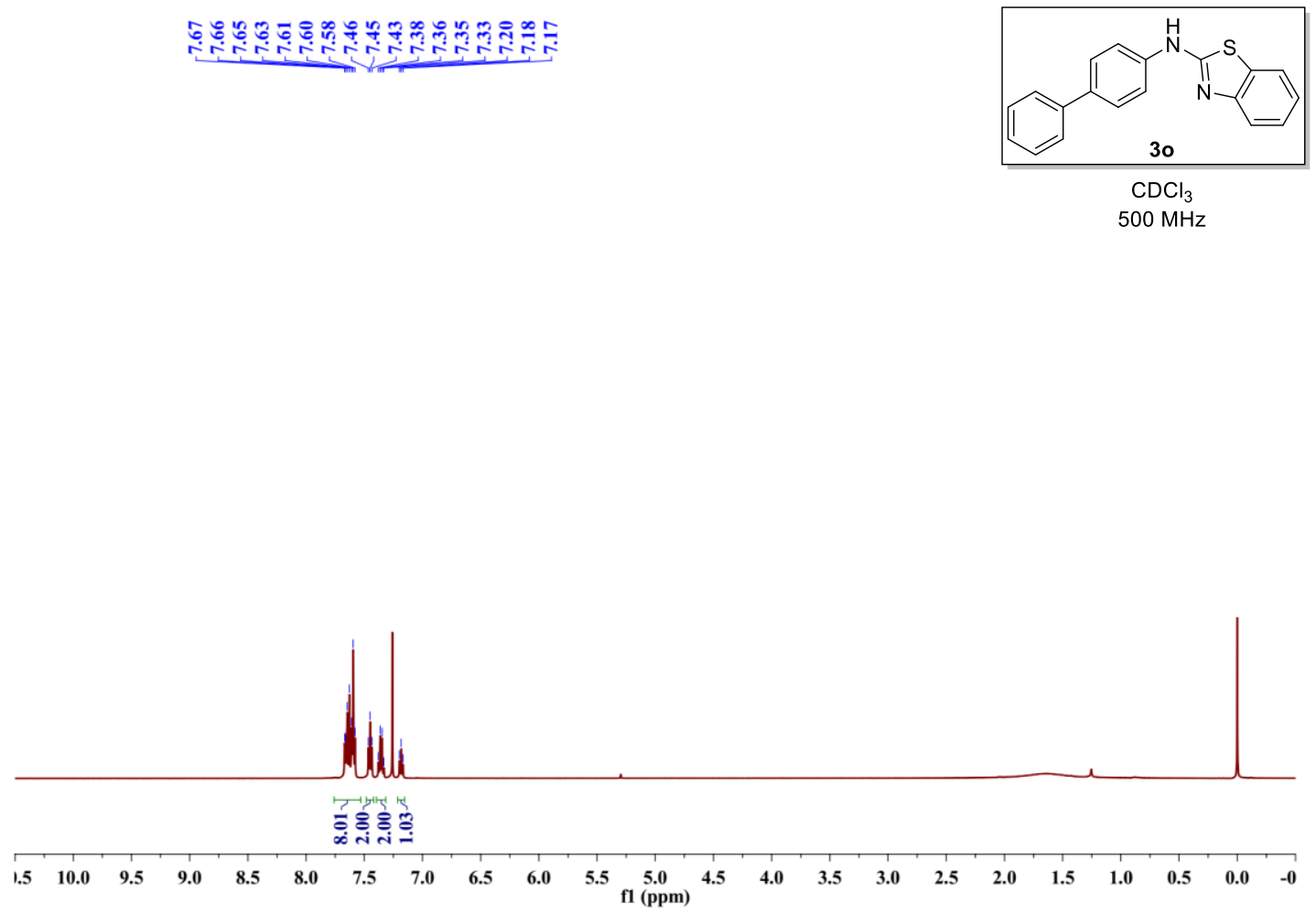

$>{ }^{13} \mathrm{C}$ NMR spectrum for 30

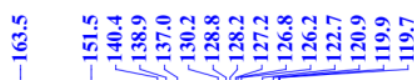

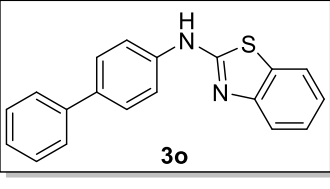

$\mathrm{CDCl}_{3}$ $126 \mathrm{MHz}$

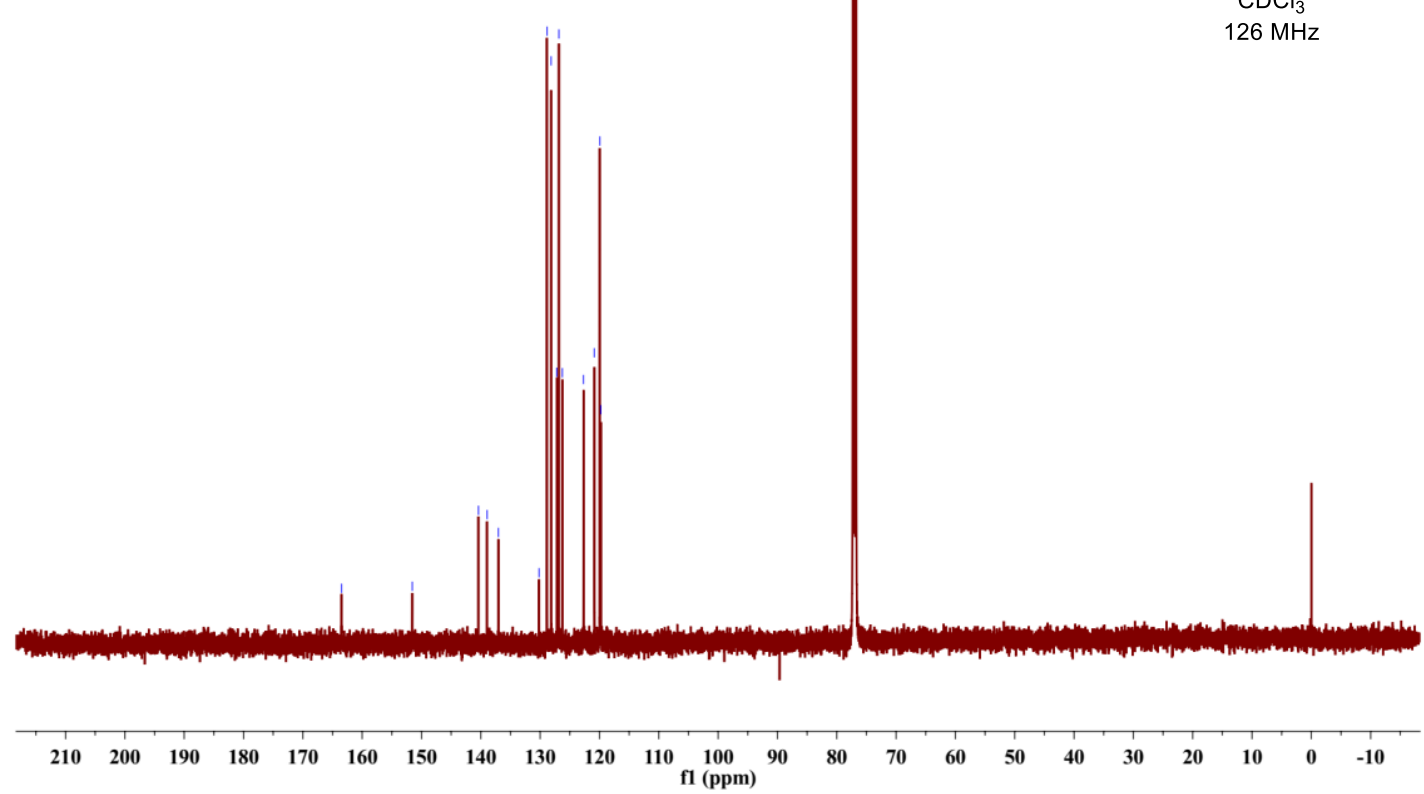


${ }^{1} \mathrm{H}$ NMR spectrum for $\mathbf{3 p}$

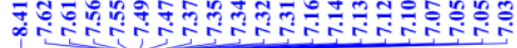

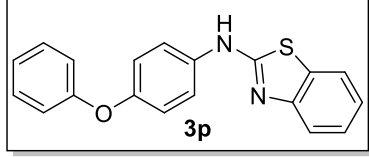

$\mathrm{CDCl}_{3}$ $500 \mathrm{MHz}$

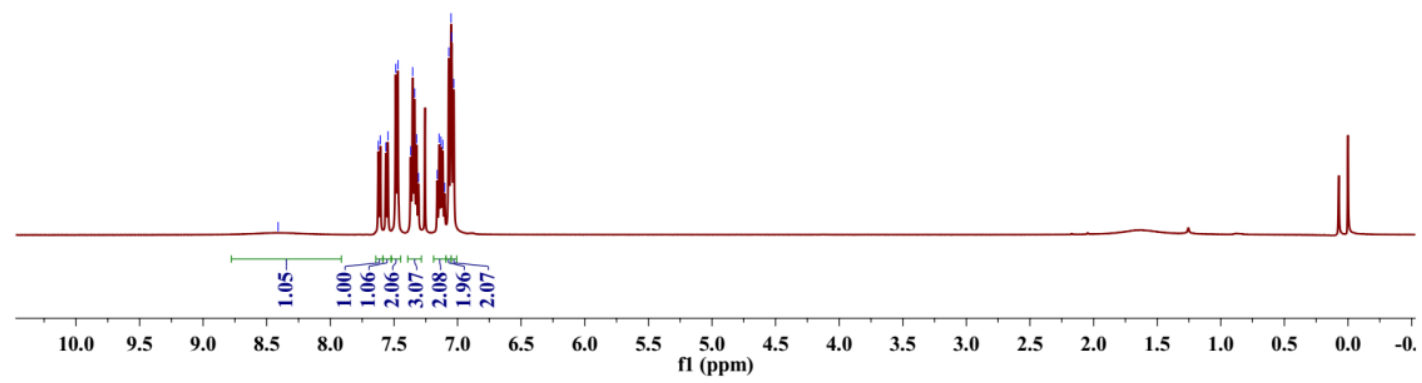

$>{ }^{13} \mathrm{C}$ NMR spectrum for $\mathbf{3 p}$

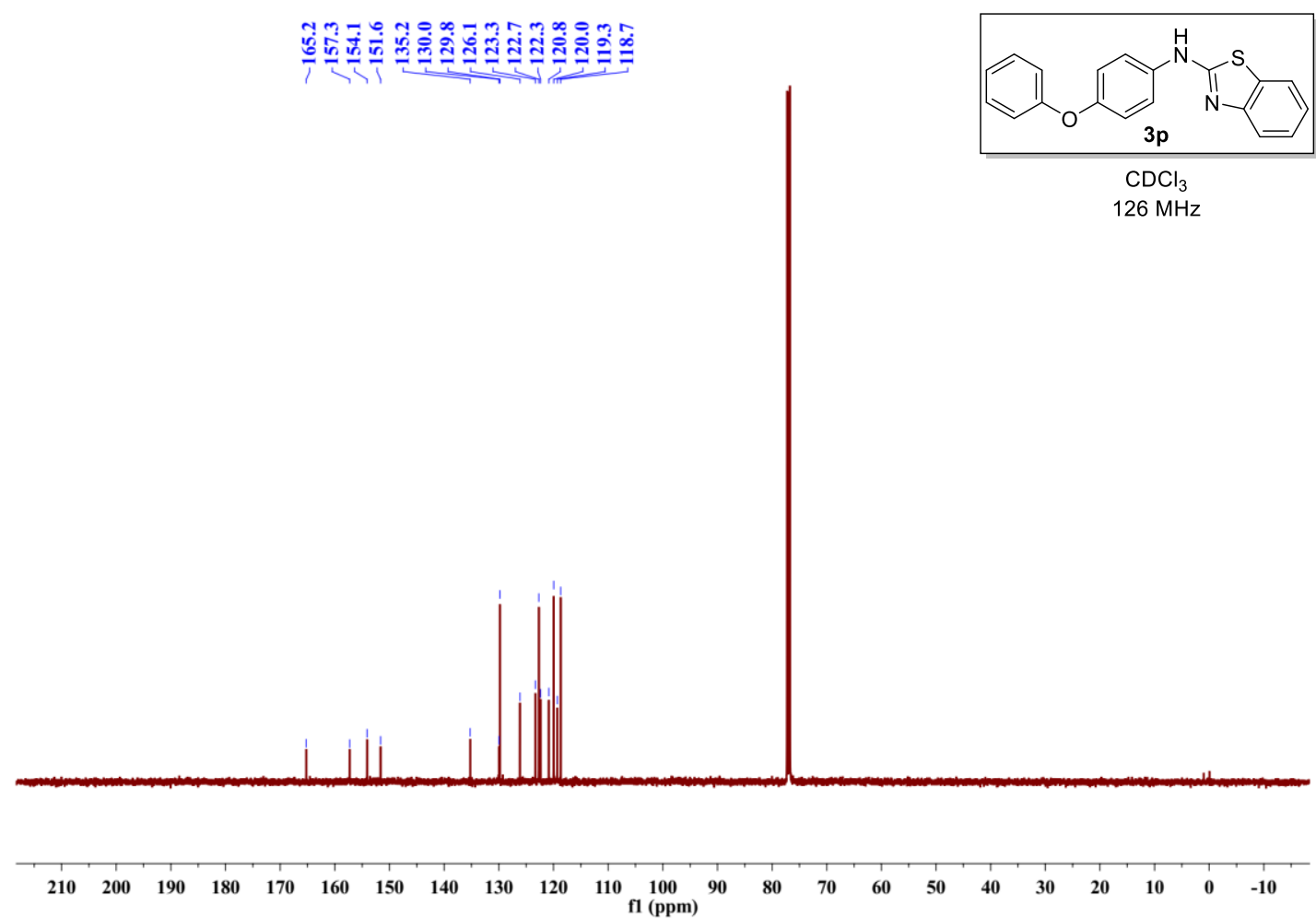


${ }^{1} \mathrm{H}$ NMR spectrum for $\mathbf{3 q}$

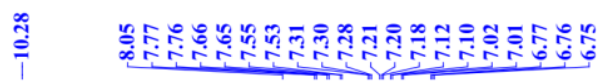
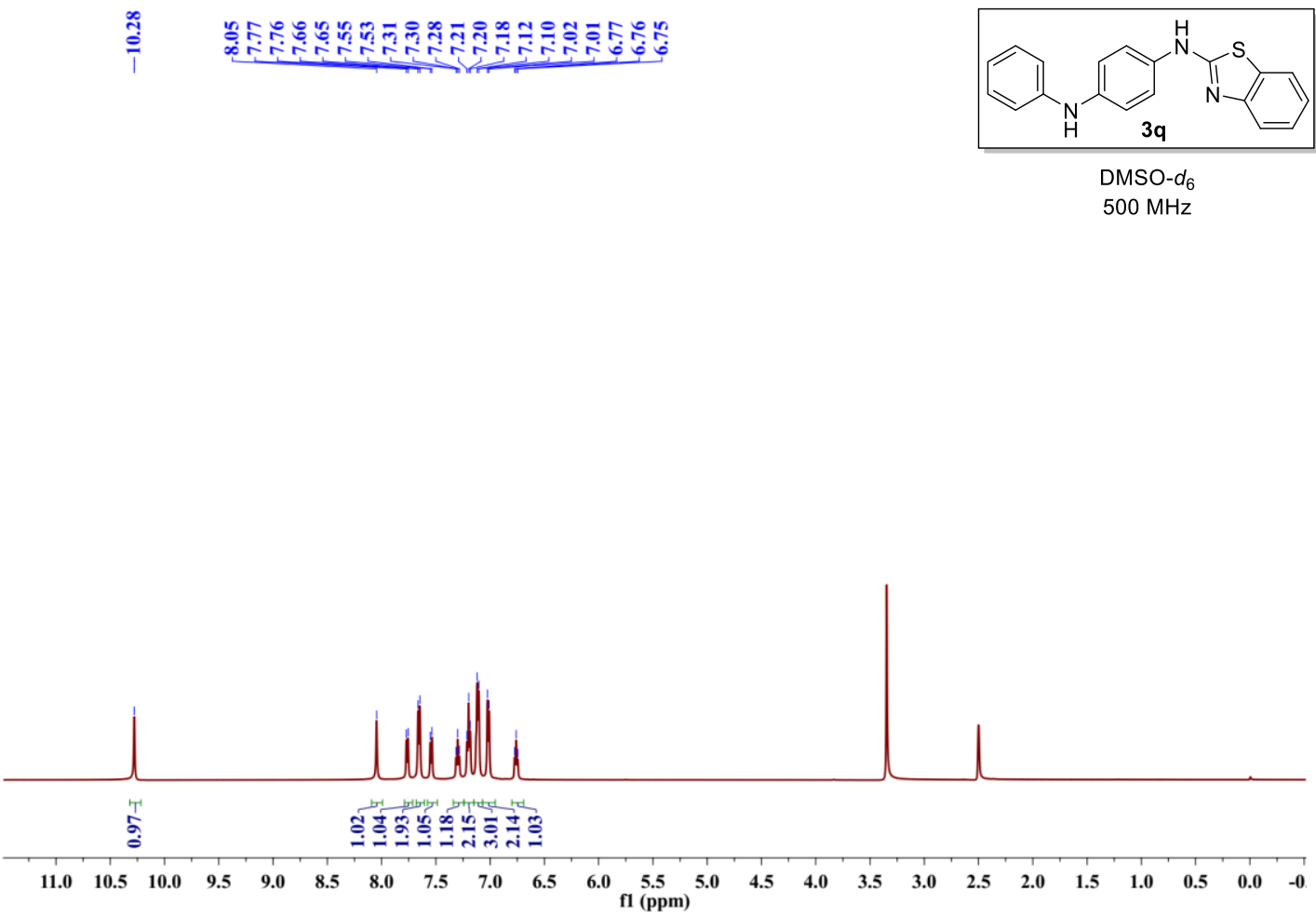

$>{ }^{13} \mathrm{C}$ NMR spectrum for $\mathbf{3 q}$

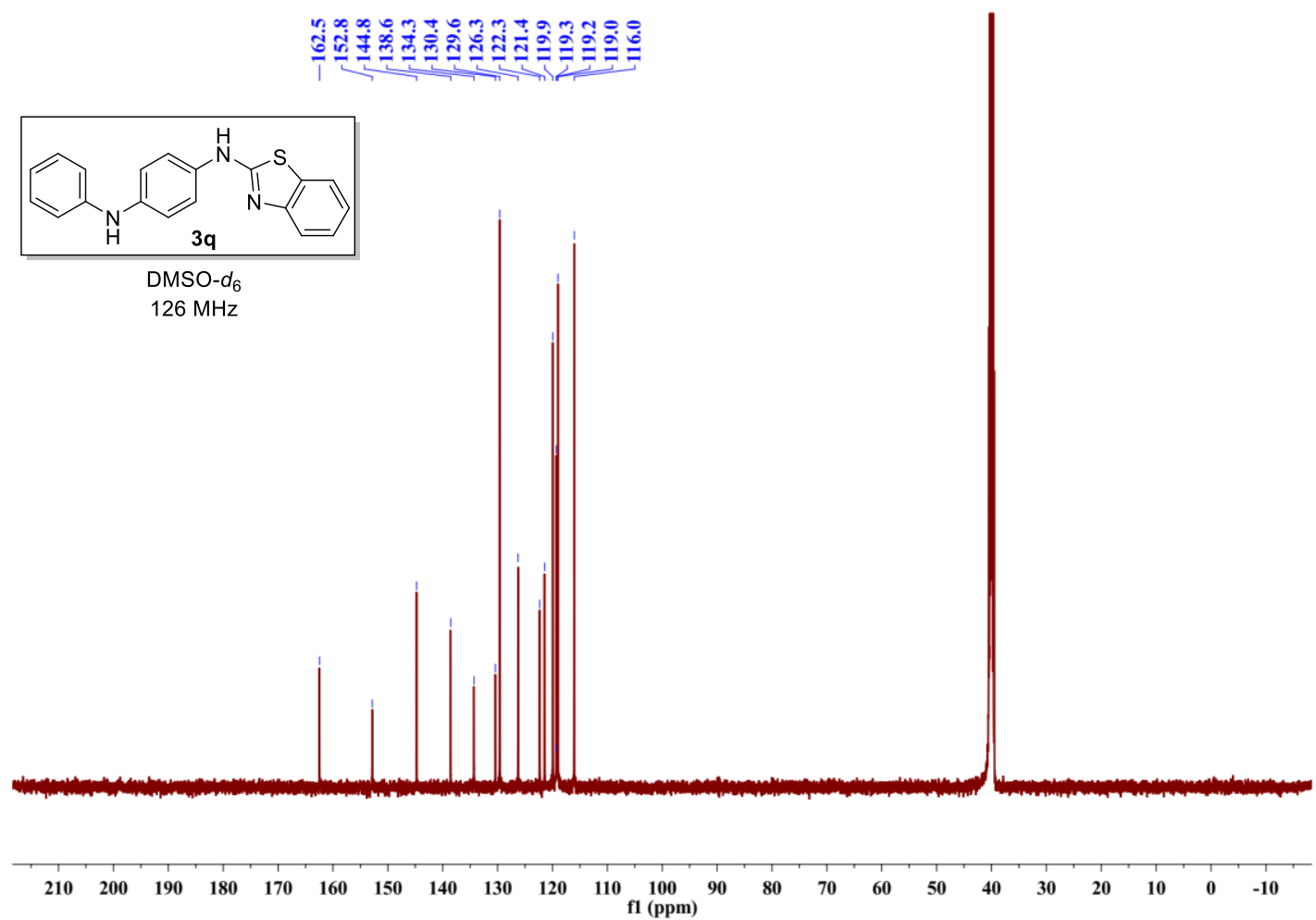


${ }^{1} \mathrm{H}$ NMR spectrum for $\mathbf{3 r}$
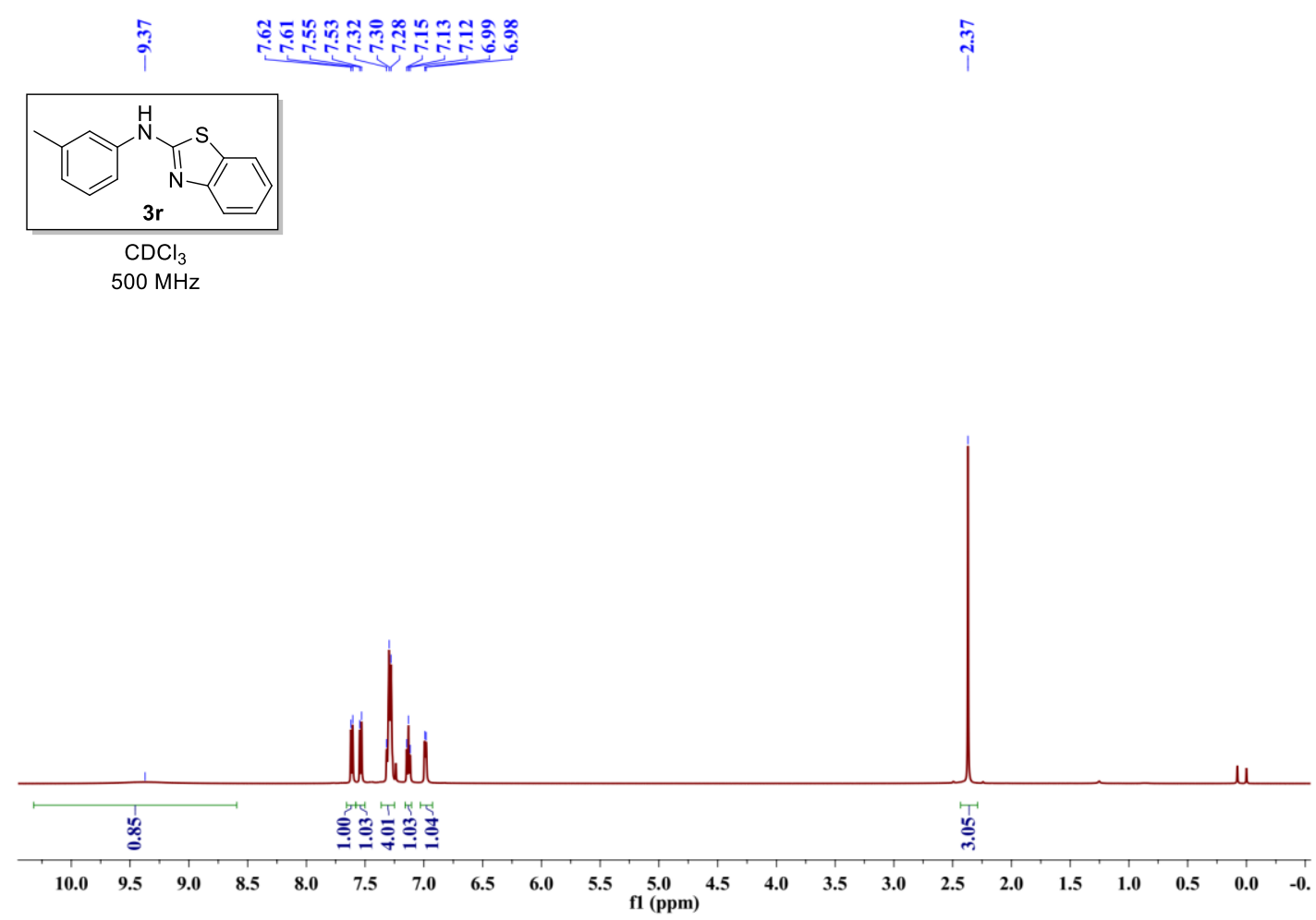

$>{ }^{13} \mathrm{C}$ NMR spectrum for $\mathbf{3 r}$

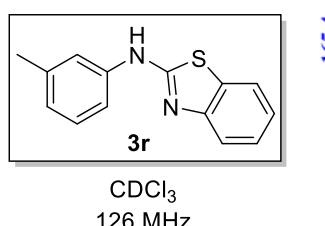

菖

$\stackrel{\text { in }}{\frac{1}{1}}$

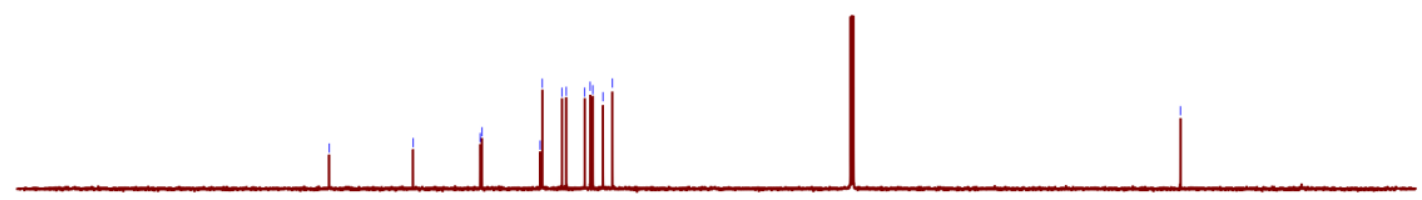

$\begin{array}{lllllllllllllllllllllllll}210 & 200 & 190 & 180 & 170 & 160 & 150 & 140 & 130 & 120 & 110 & 100 & 90 & 80 & 70 & 60 & 50 & 40 & 30 & 20 & 10 & 0 & -10\end{array}$ 
${ }^{1} \mathrm{H}$ NMR spectrum for $\mathbf{3 s}$
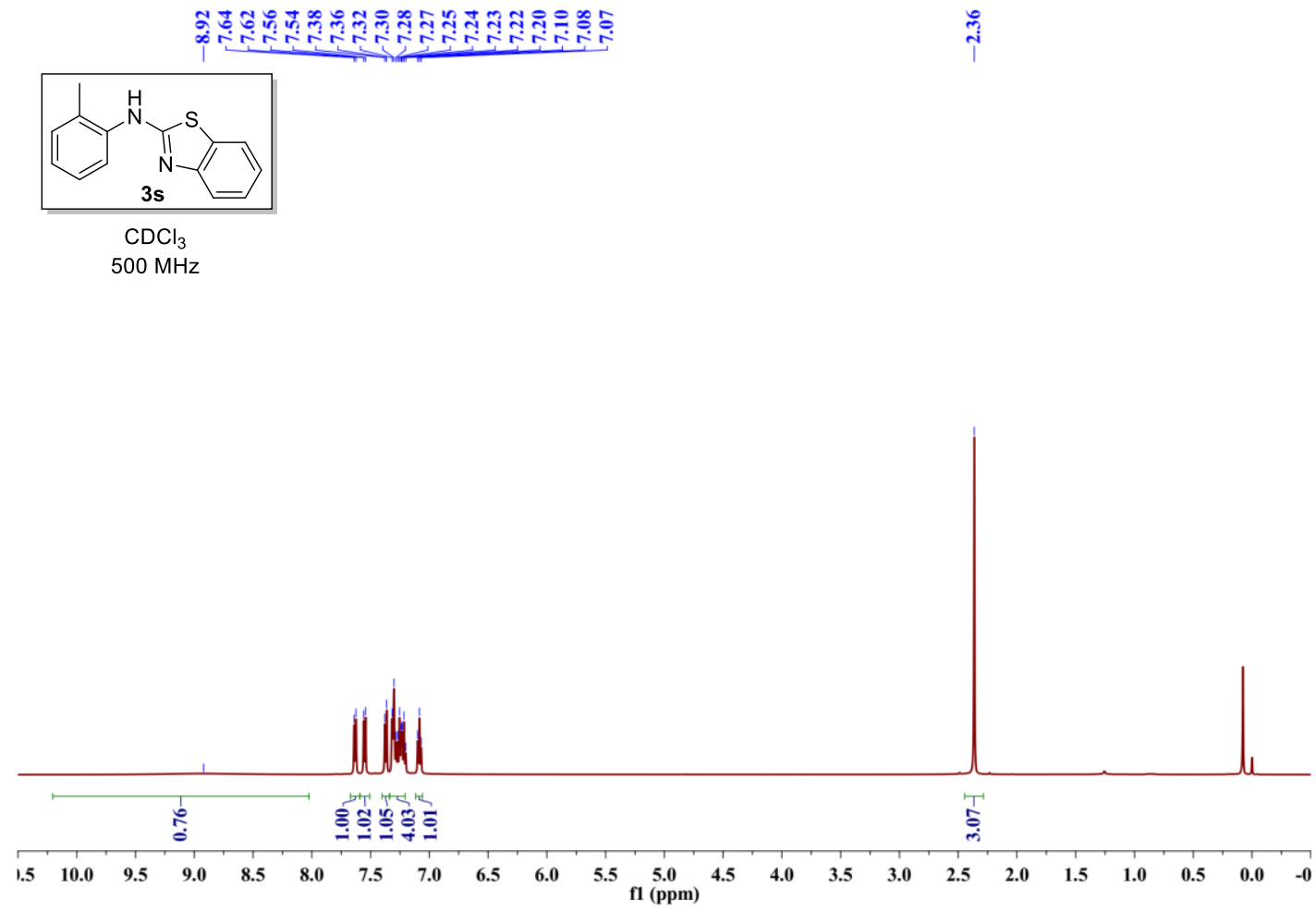

$>{ }^{13} \mathrm{C}$ NMR spectrum for $3 \mathrm{~s}$

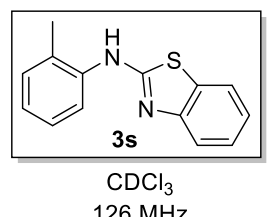

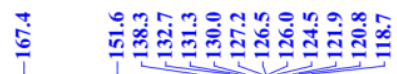

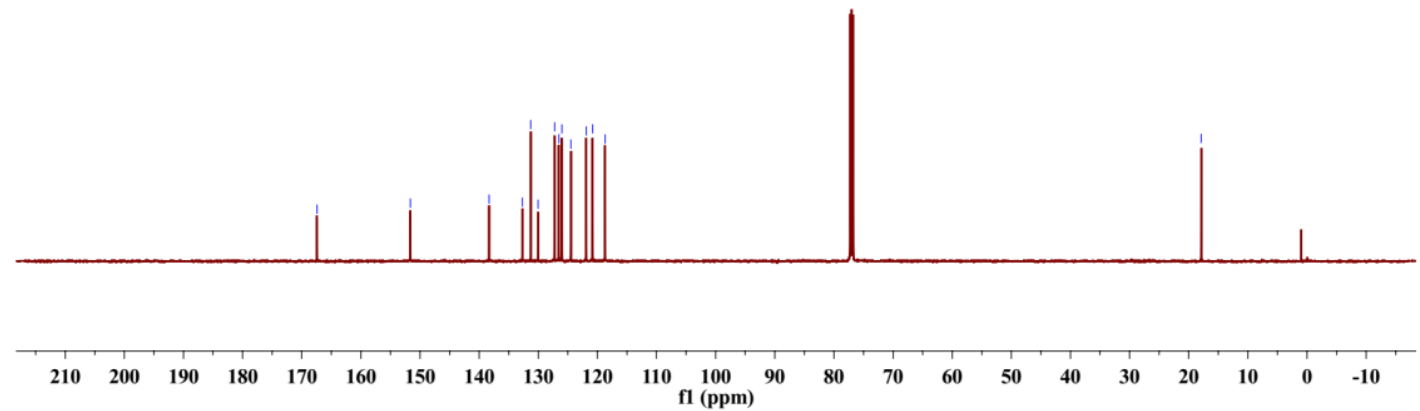


${ }^{1} \mathrm{H}$ NMR spectrum for $\mathbf{3 t}$

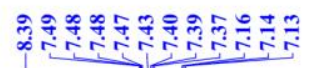
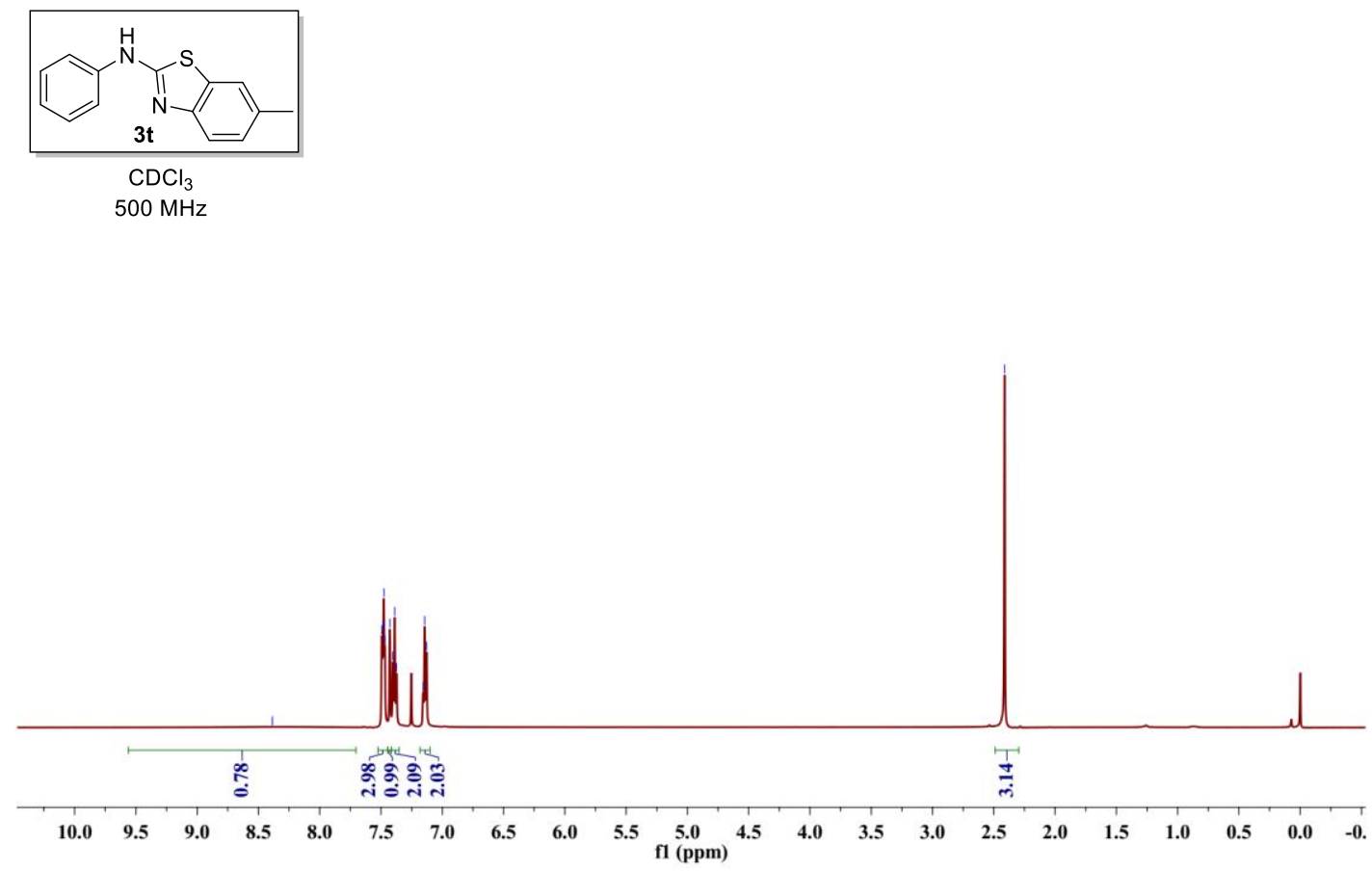

${ }^{13} \mathrm{C}$ NMR spectrum for $\mathbf{3 t}$

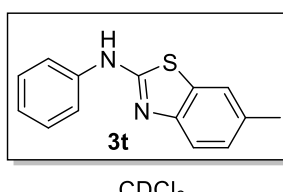

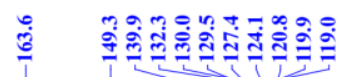

$\frac{m}{2}$

$126 \mathrm{MHz}$

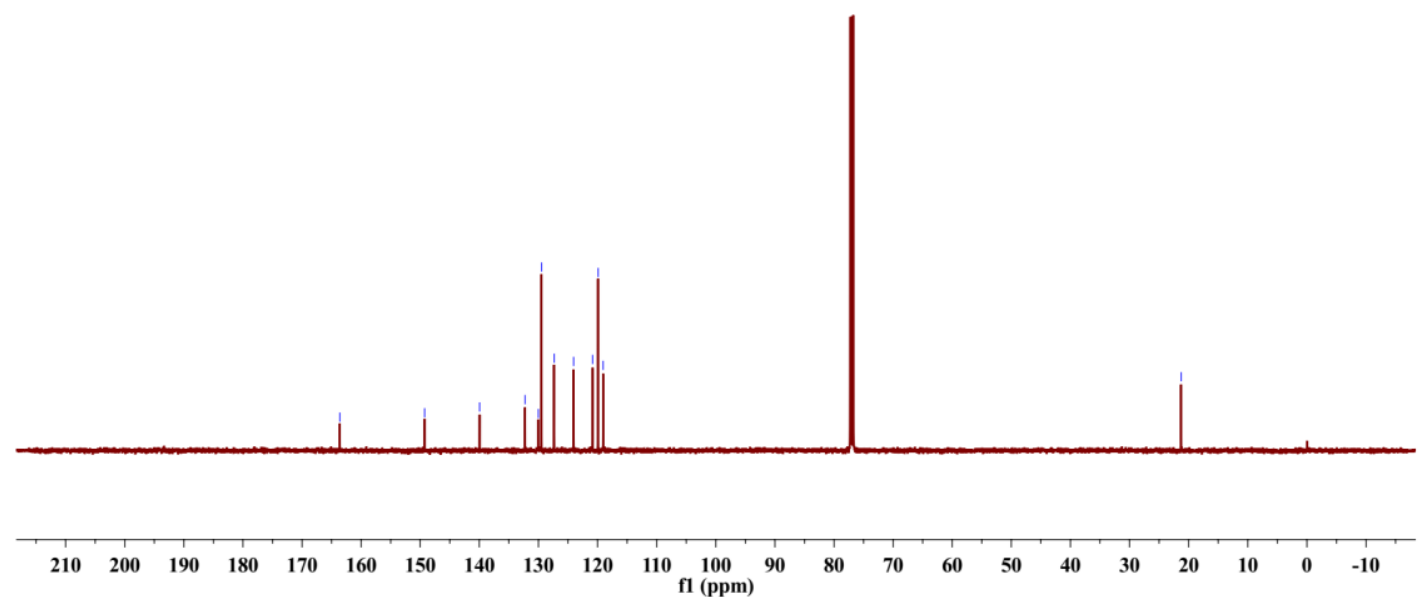


${ }^{1} \mathrm{H}$ NMR spectrum for $\mathbf{3 u}$
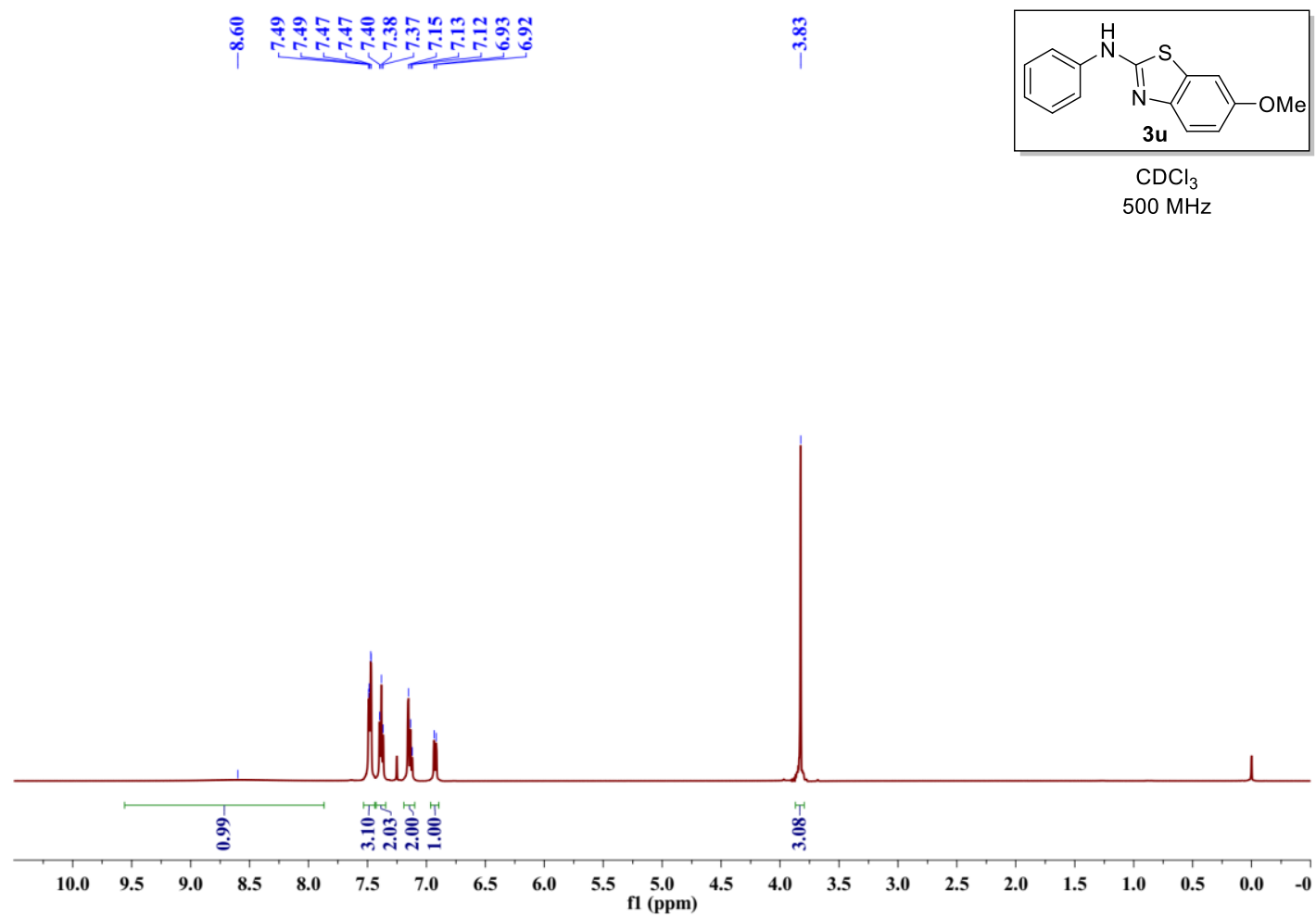

$>{ }^{13} \mathrm{C}$ NMR spectrum for $\mathbf{3 u}$

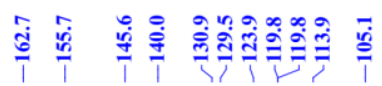

î

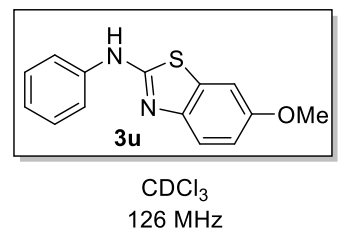

$126 \mathrm{MHz}$

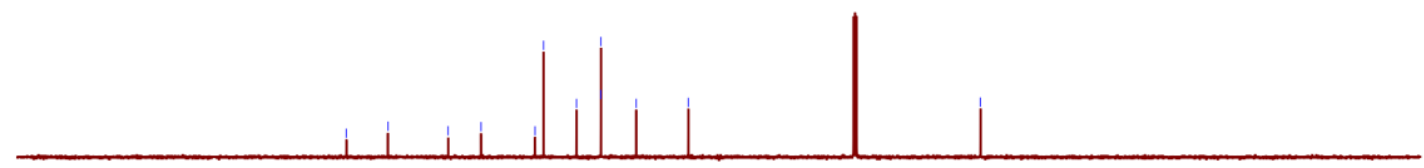

$\begin{array}{lllllllllllllllllllllll}210 & 200 & 190 & 180 & 170 & 160 & 150 & 140 & 130 & 120 & 110 & \begin{array}{c}100 \\ \mathrm{fl}(\mathrm{ppm})\end{array} & 90 & 80 & 70 & 60 & 50 & 40 & 30 & 20 & 10 & 0 & -10\end{array}$ 
${ }^{1} \mathrm{H}$ NMR spectrum for $\mathbf{3 v}$

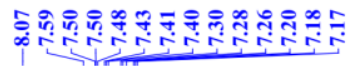
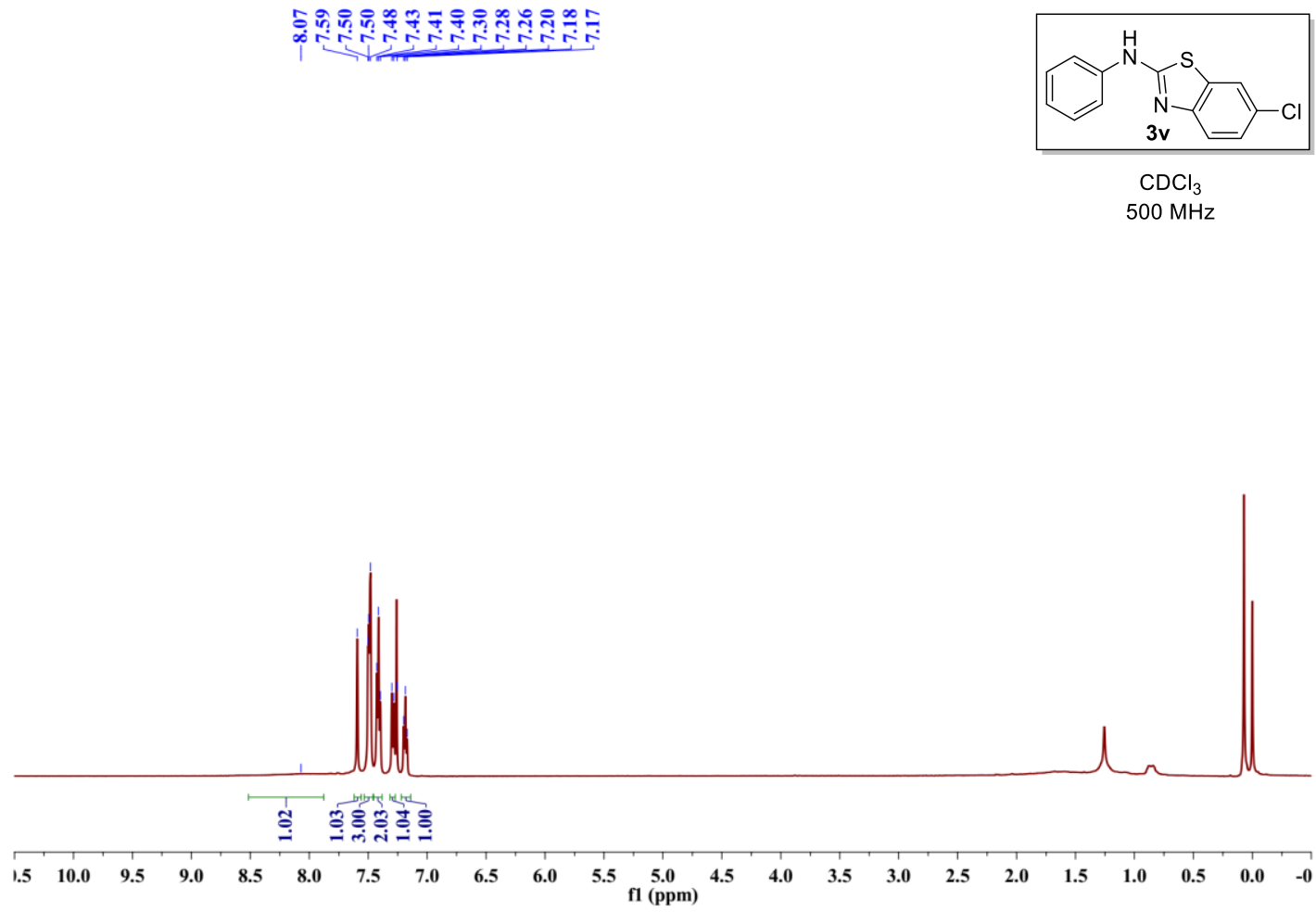

$>{ }^{13} \mathrm{C}$ NMR spectrum for $\mathbf{3 v}$

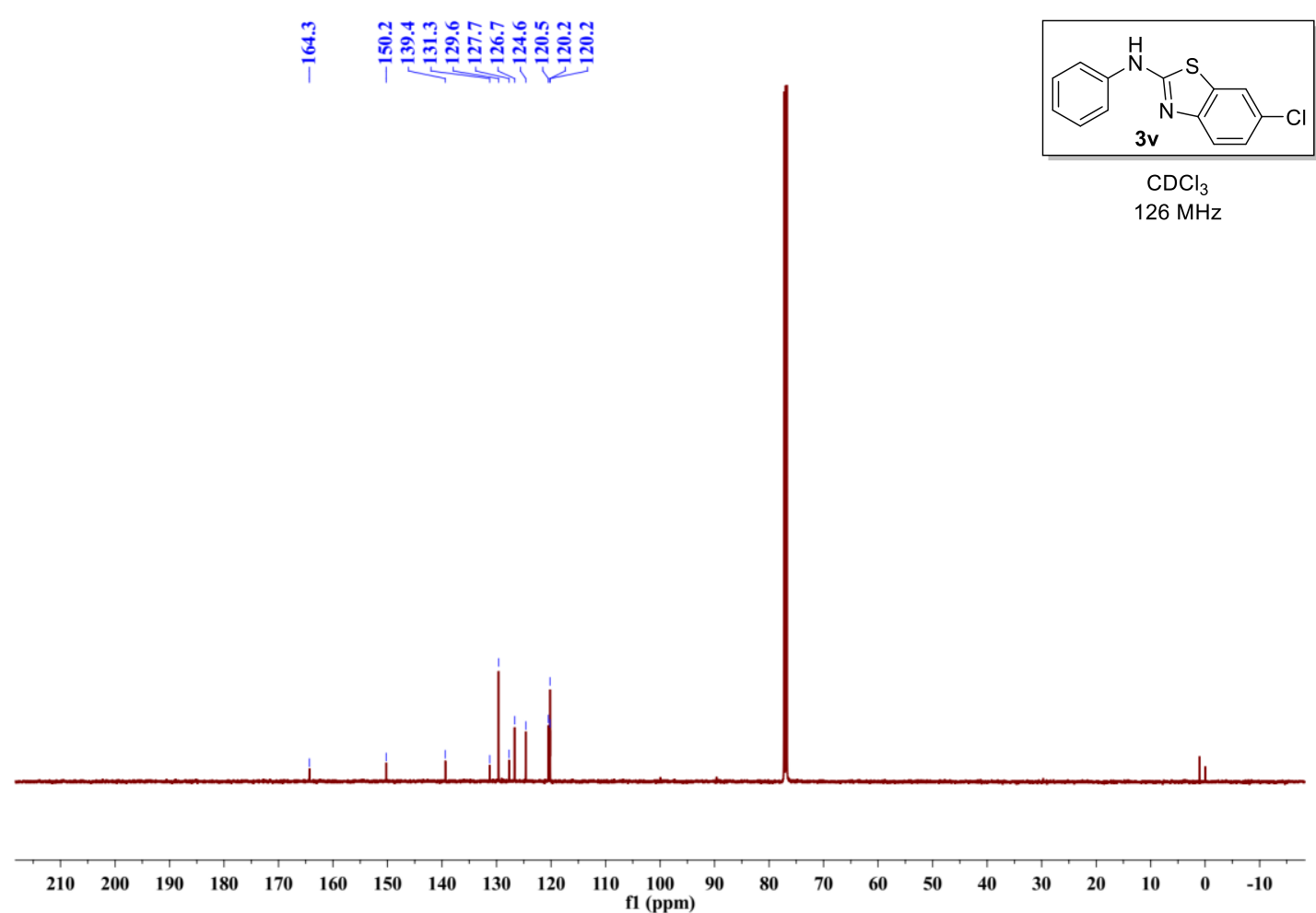


${ }^{1} \mathrm{H}$ NMR spectrum for $\mathbf{3 w}$

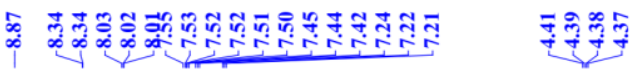

華导

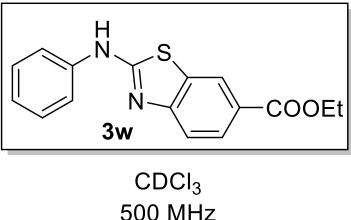

$500 \mathrm{MHz}$

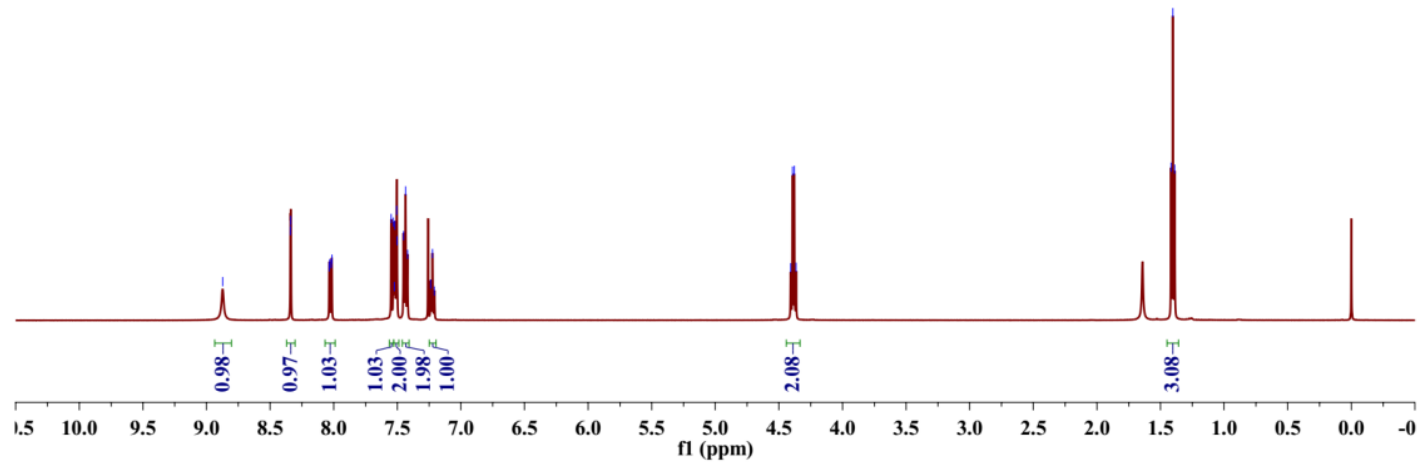

$>{ }^{13} \mathrm{C}$ NMR spectrum for $3 w$

毠

今े
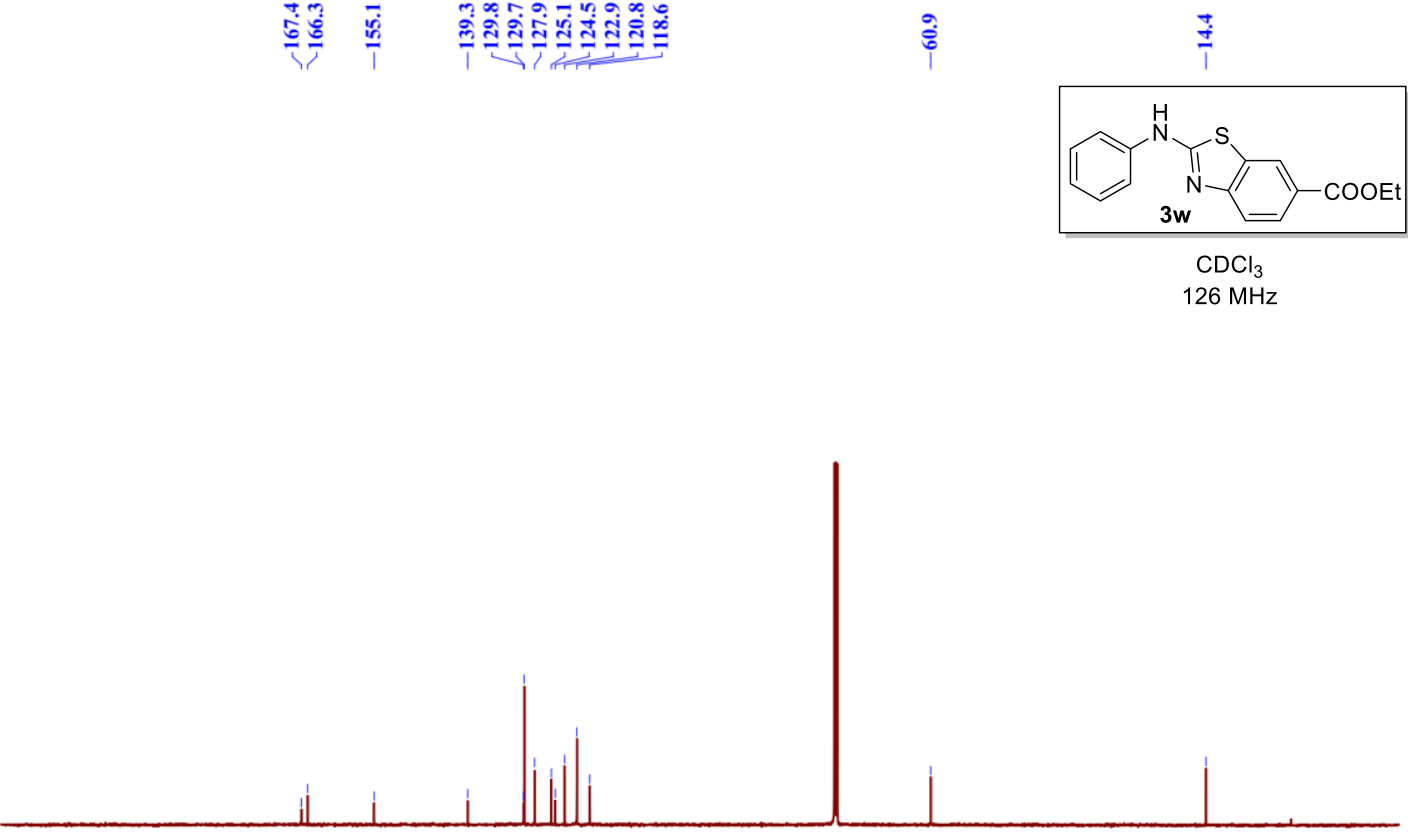

$\begin{array}{lllllllllllllllllllllll}210 & 200 & 190 & 180 & 170 & 160 & 150 & 140 & 130 & 120 & 110 & 100 & 90 & 80 & 70 & 60 & 50 & 40 & 30 & 20 & 10 & 0 & -10\end{array}$ 
${ }^{1} \mathrm{H}$ NMR spectrum for $\mathbf{3 x}$

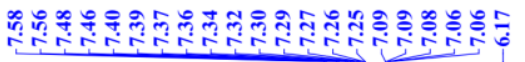

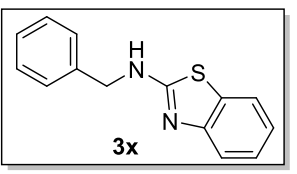

$\mathrm{CDCl}_{3}$ $500 \mathrm{MHz}$

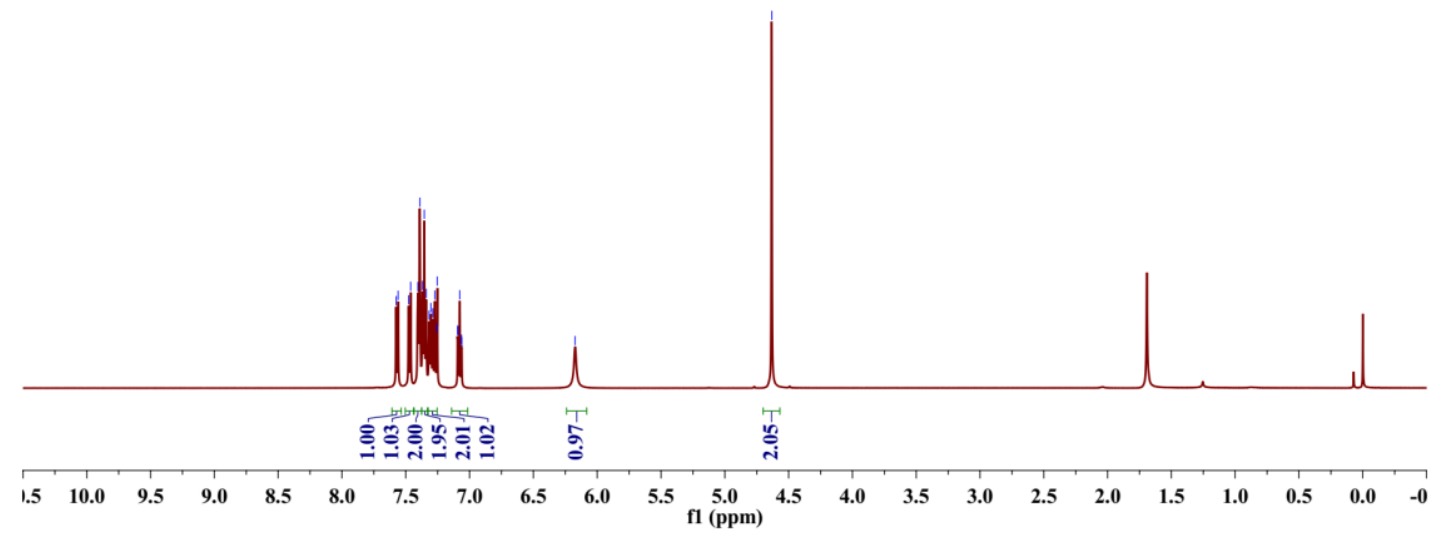

$>{ }^{13} \mathrm{C}$ NMR spectrum for $\mathbf{3 x}$

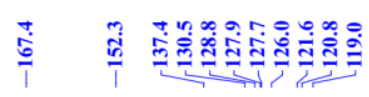
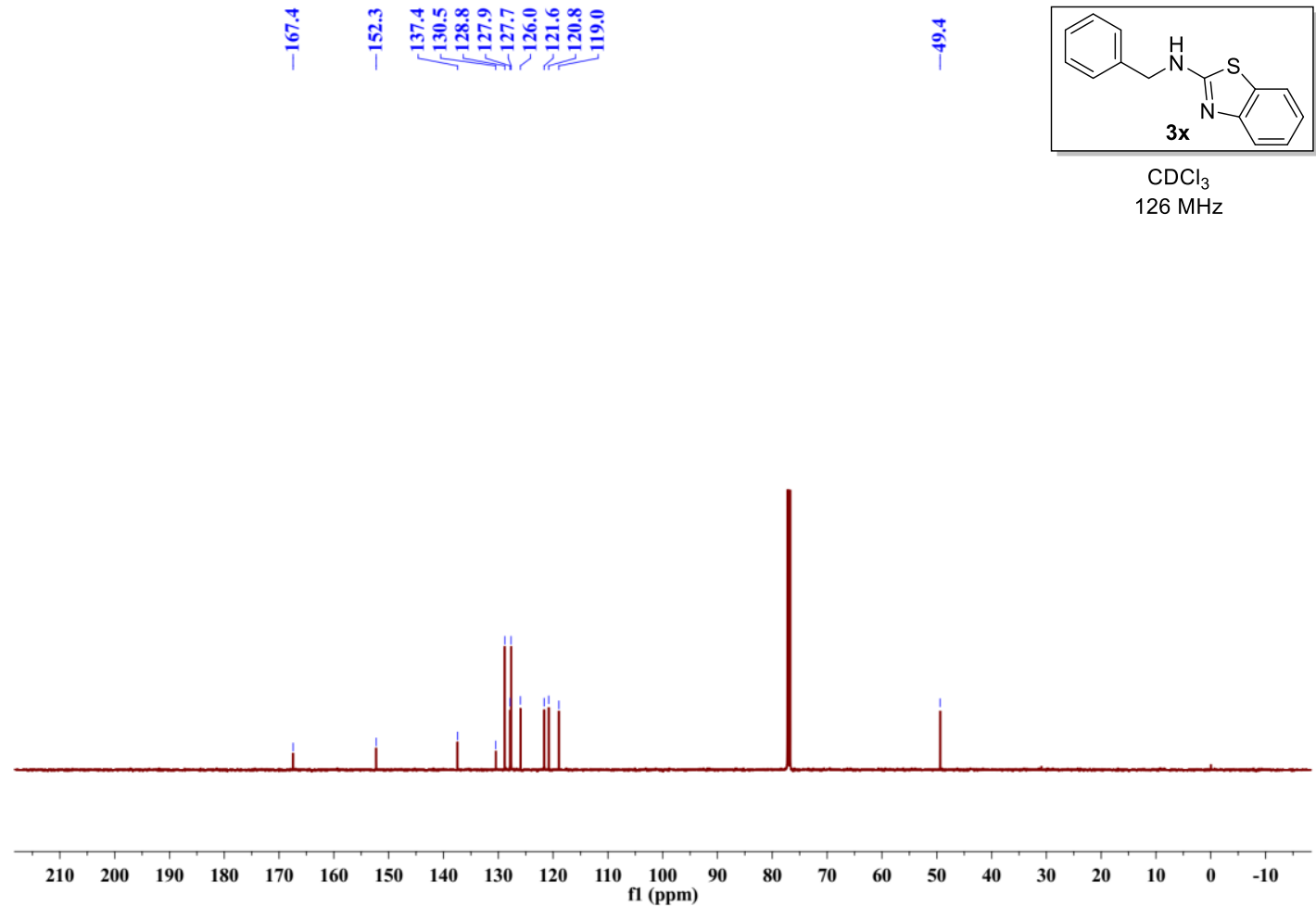
${ }^{1} \mathrm{H}$ NMR spectrum for $\mathbf{3 y}$

月.

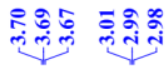
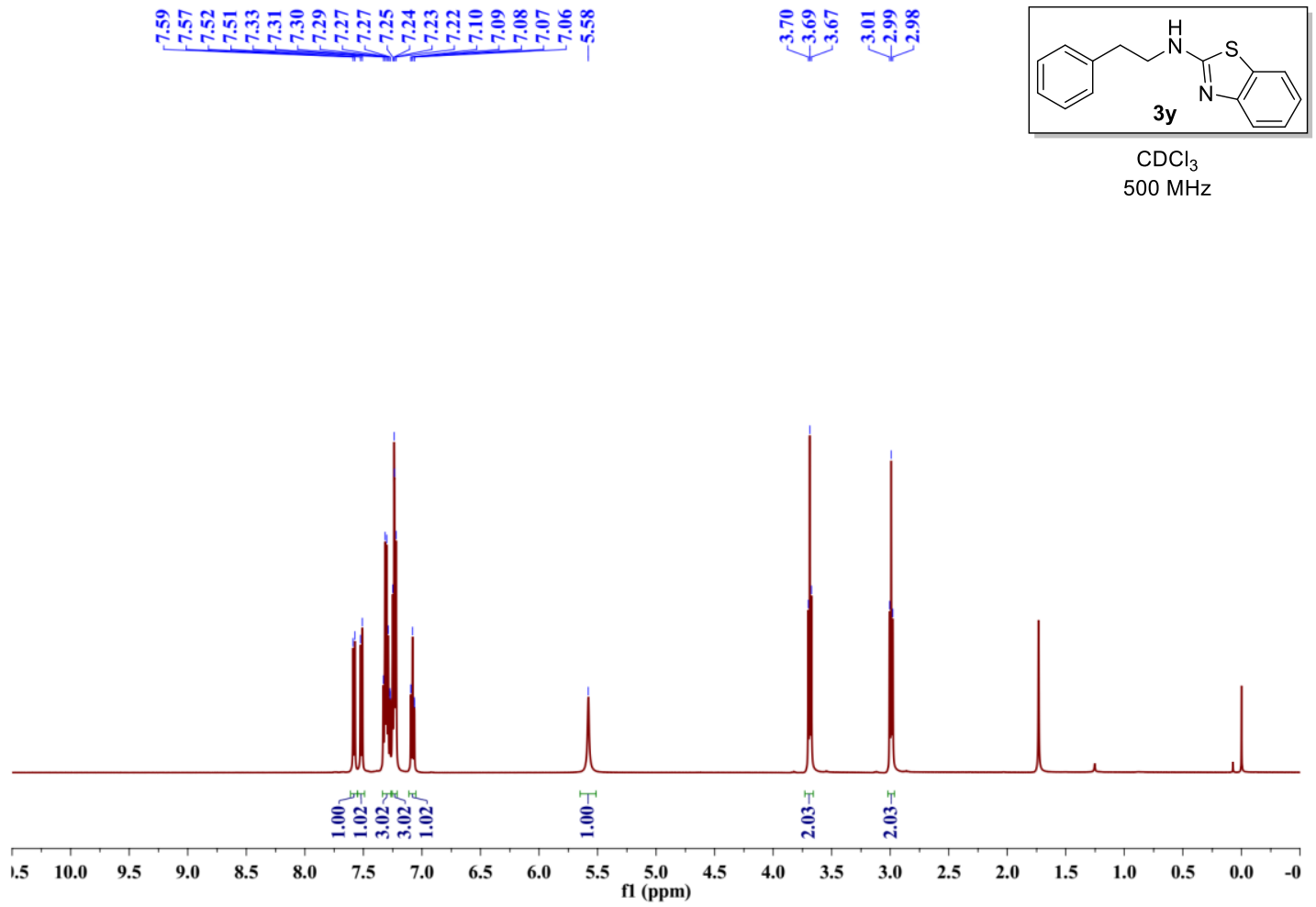

$>{ }^{13} \mathrm{C}$ NMR spectrum for $\mathbf{3 y}$

1

in
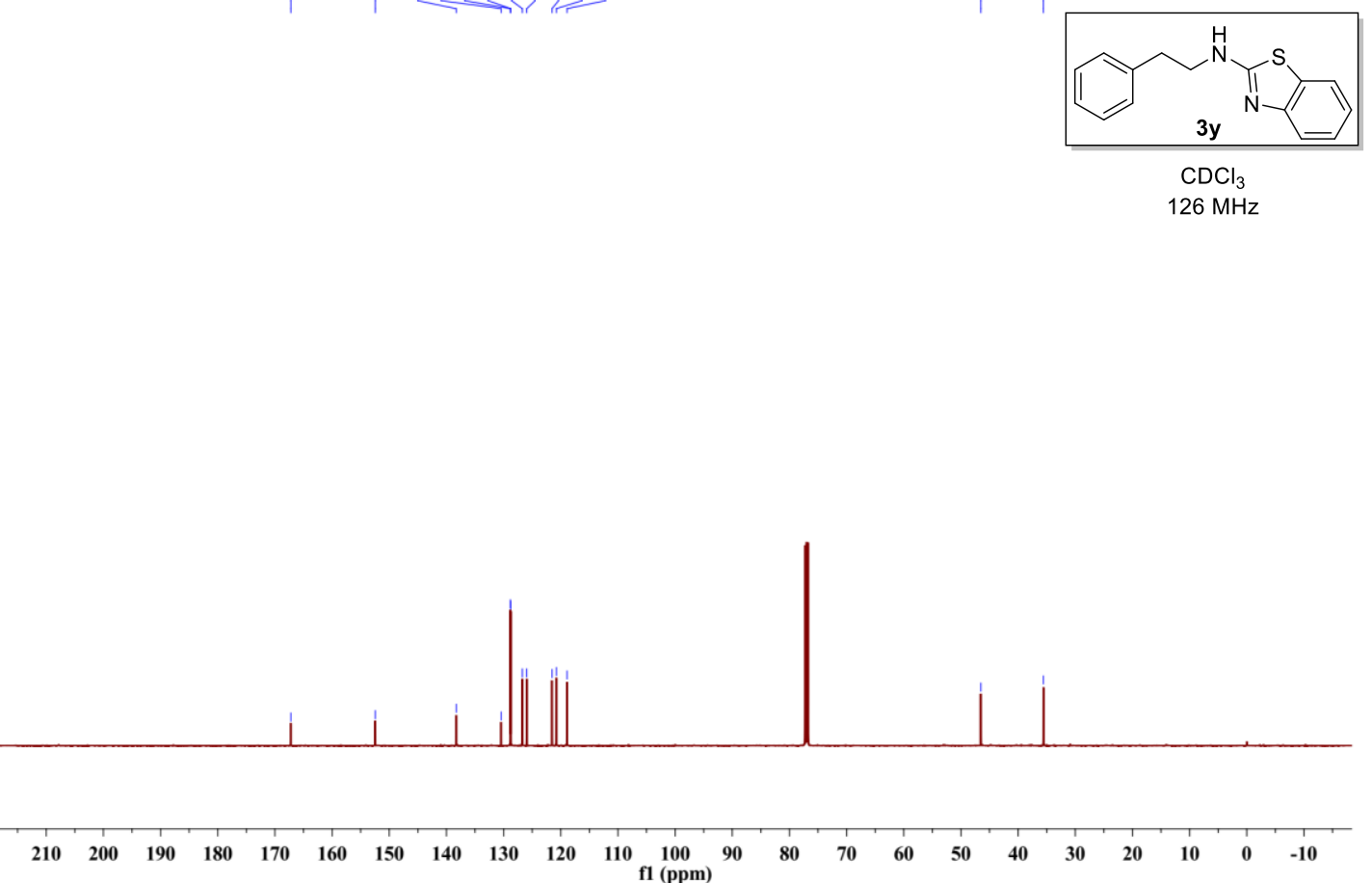
${ }^{1} \mathrm{H}$ NMR spectrum for $\mathbf{3 z}$

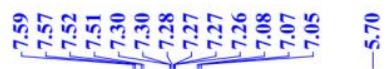

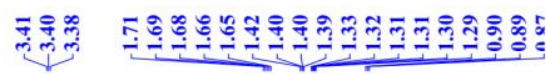

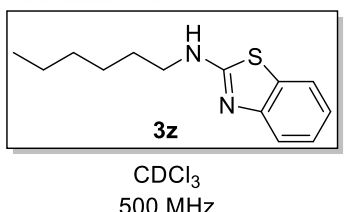

$500 \mathrm{MHz}$

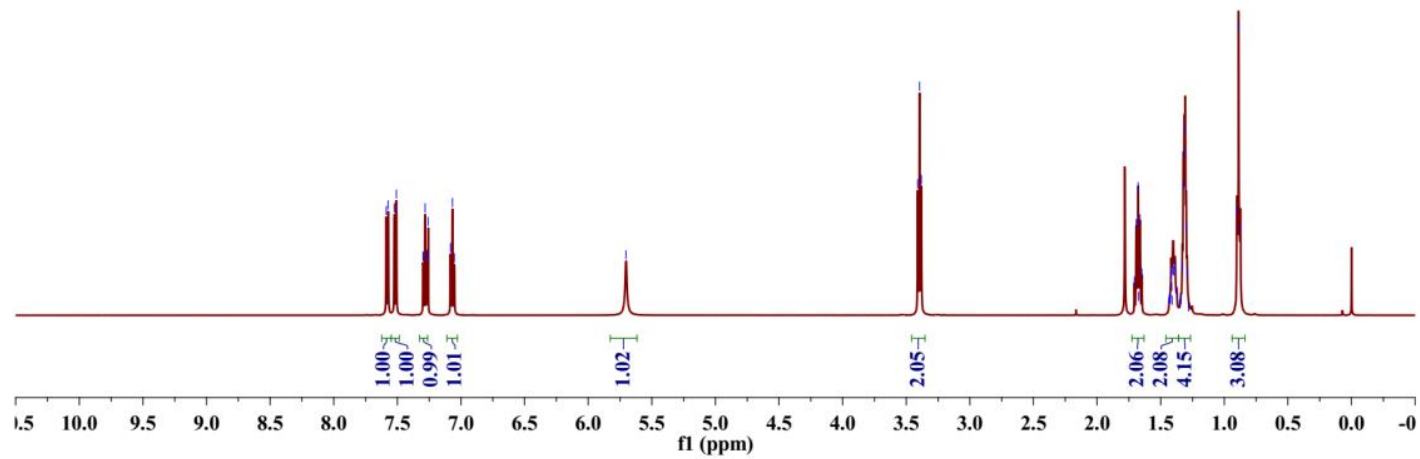

$>{ }^{13} \mathrm{C}$ NMR spectrum for $\mathbf{3 z}$

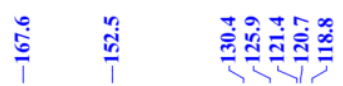

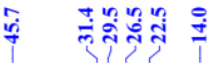
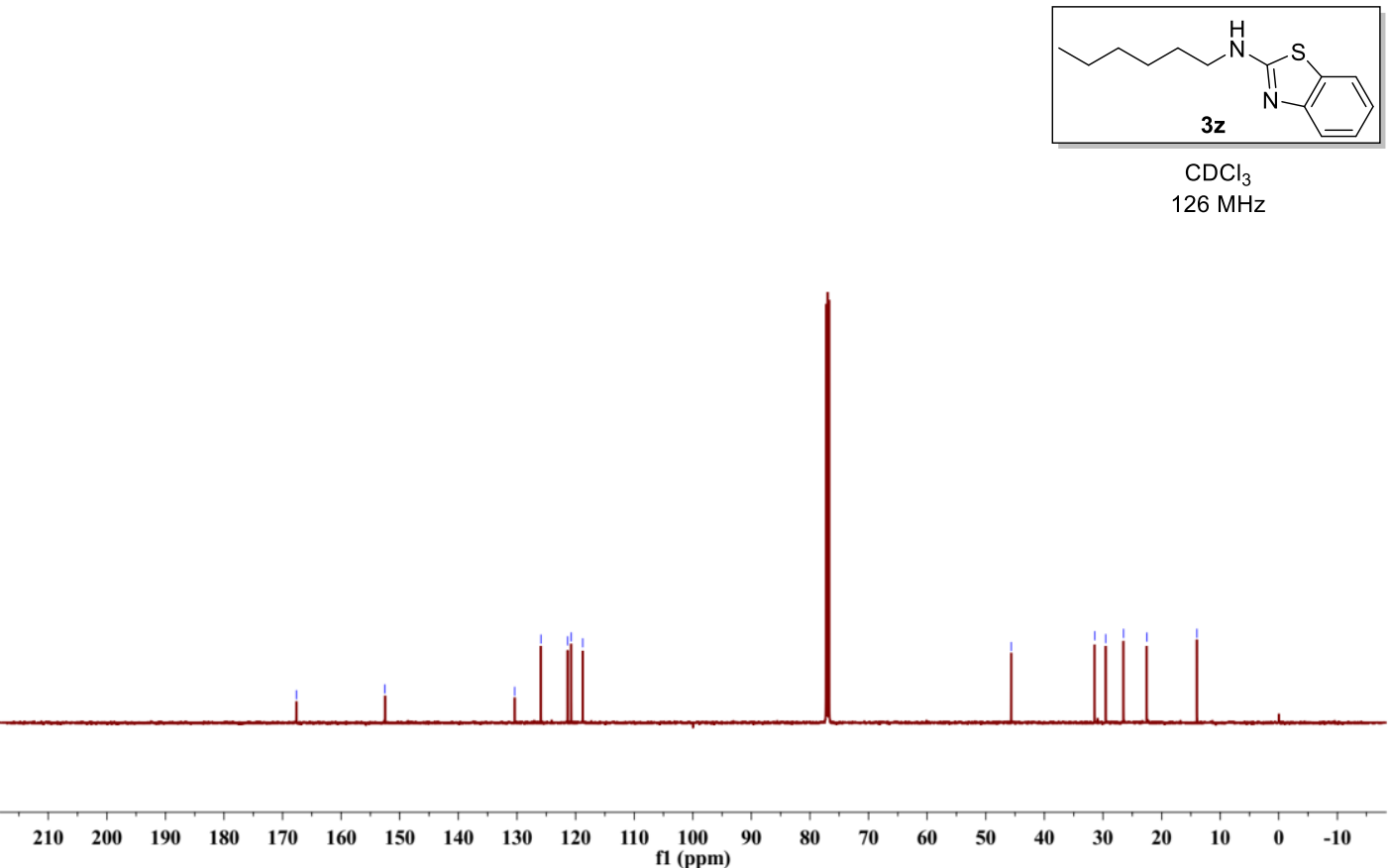
${ }^{1} \mathrm{H}$ NMR spectrum for $\mathbf{3 a}$ '

$\underbrace{1}_{1}$

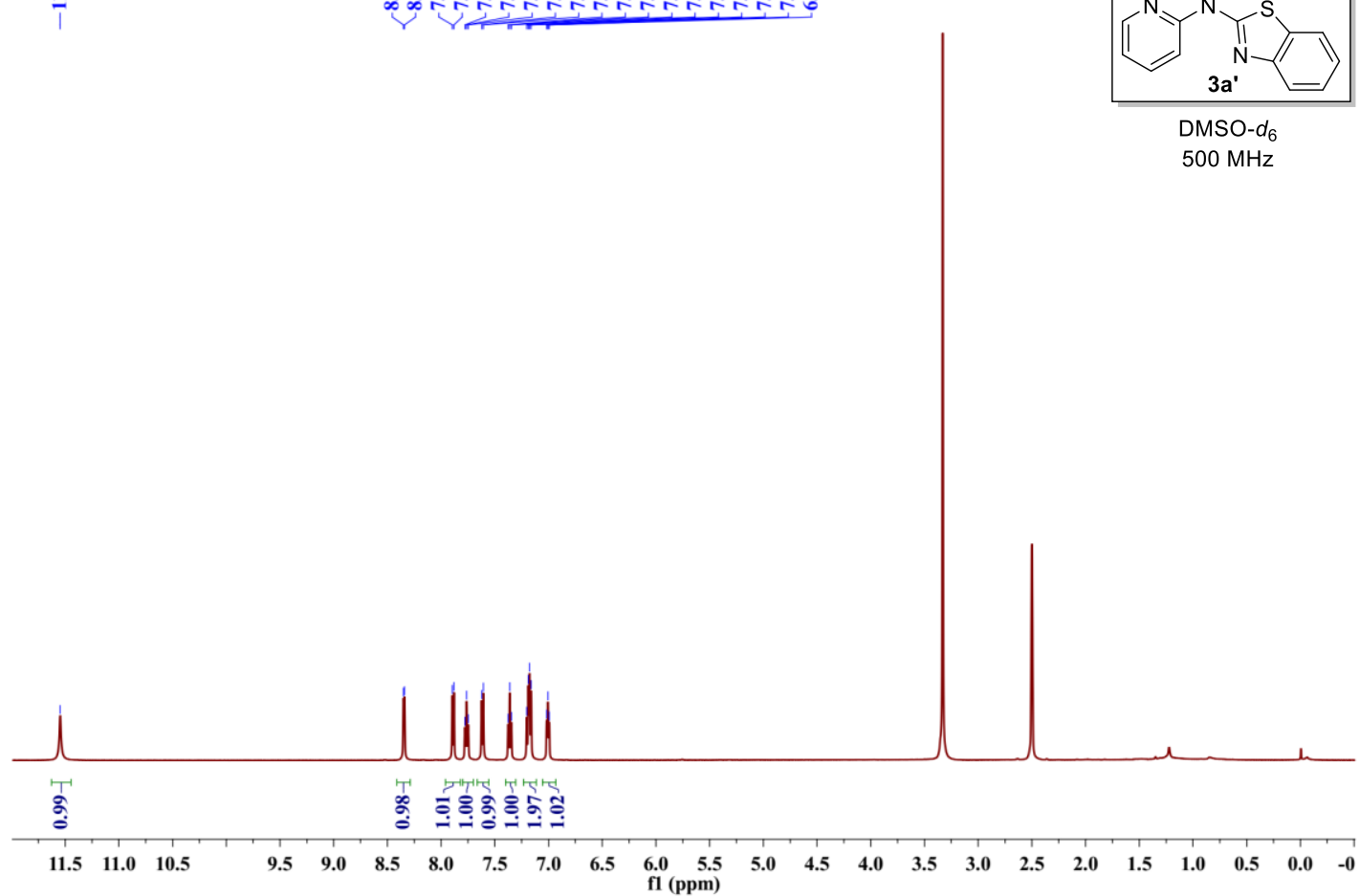

$>{ }^{13} \mathrm{C}$ NMR spectrum for $\mathbf{3 a}{ }^{\prime}$

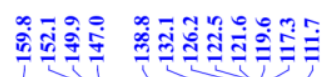

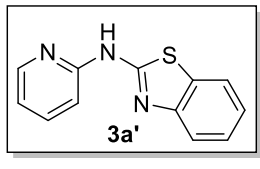

DMSO- $d_{6}$ $126 \mathrm{MHz}$

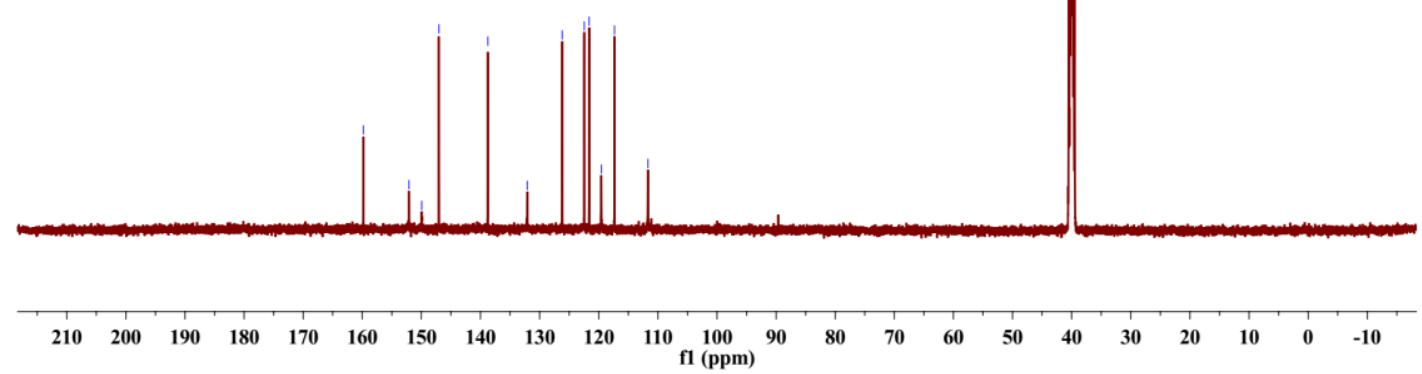


${ }^{1} \mathrm{H}$ NMR spectrum for $\mathbf{3 b}$ '

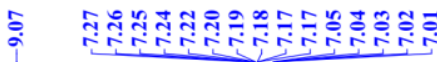

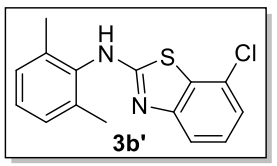

$\mathrm{CDCl}_{3}$ $500 \mathrm{MHz}$

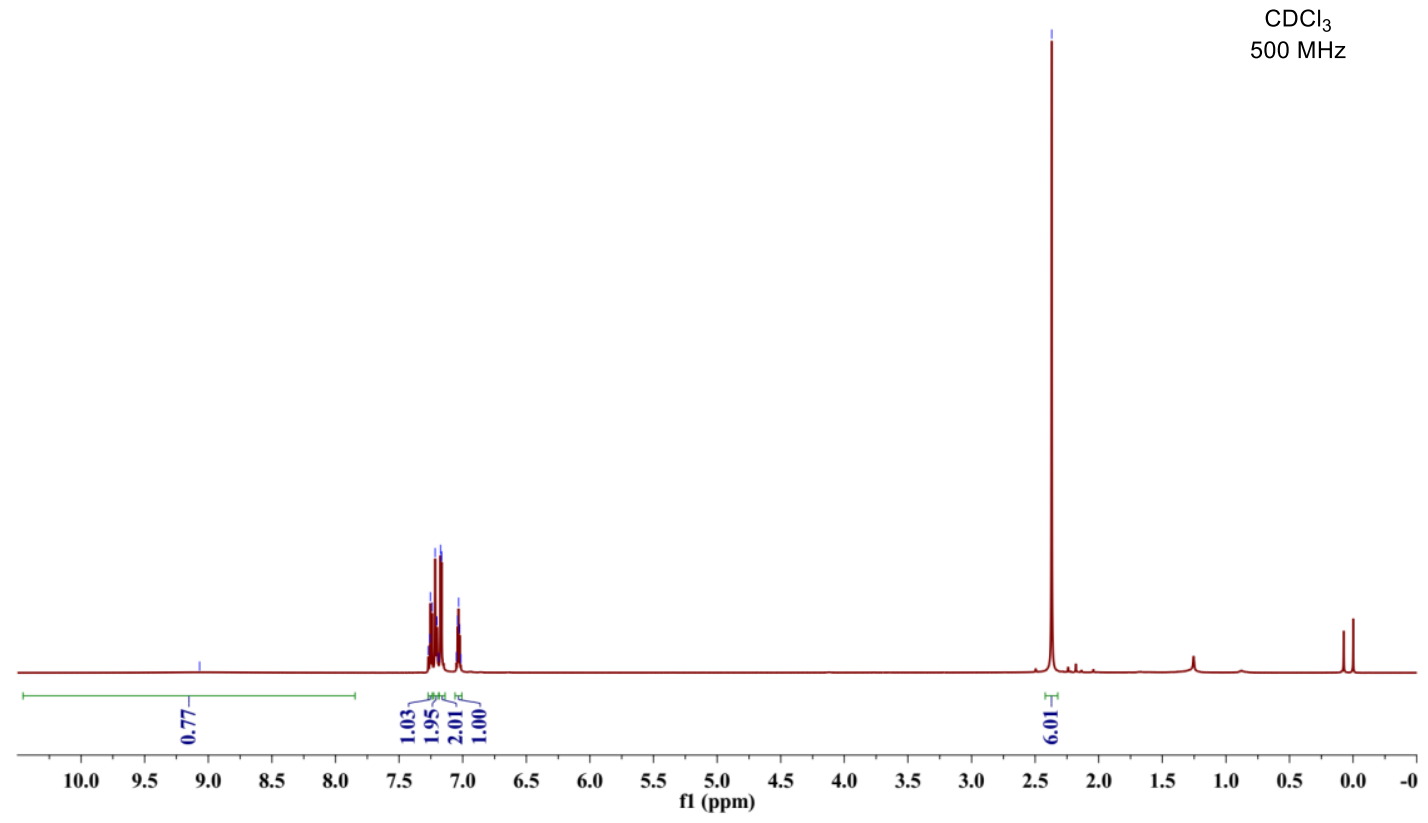

$>{ }^{13} \mathrm{C}$ NMR spectrum for $\mathbf{3 b}{ }^{\prime}$

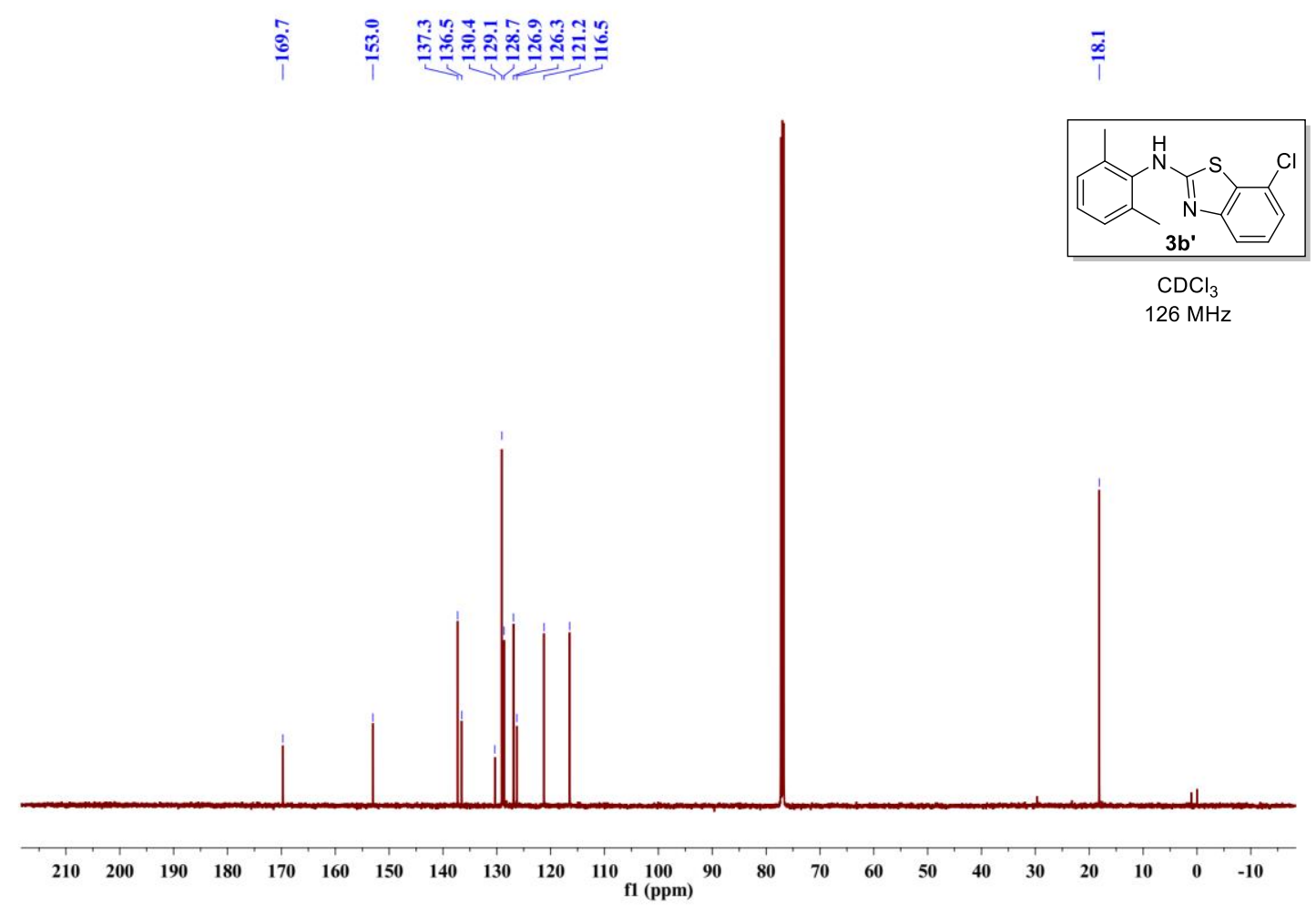


${ }^{1} \mathrm{H}$ NMR spectrum for $\mathbf{4 a}$

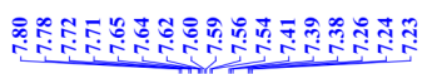

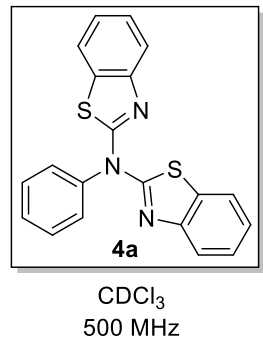

$500 \mathrm{MHz}$

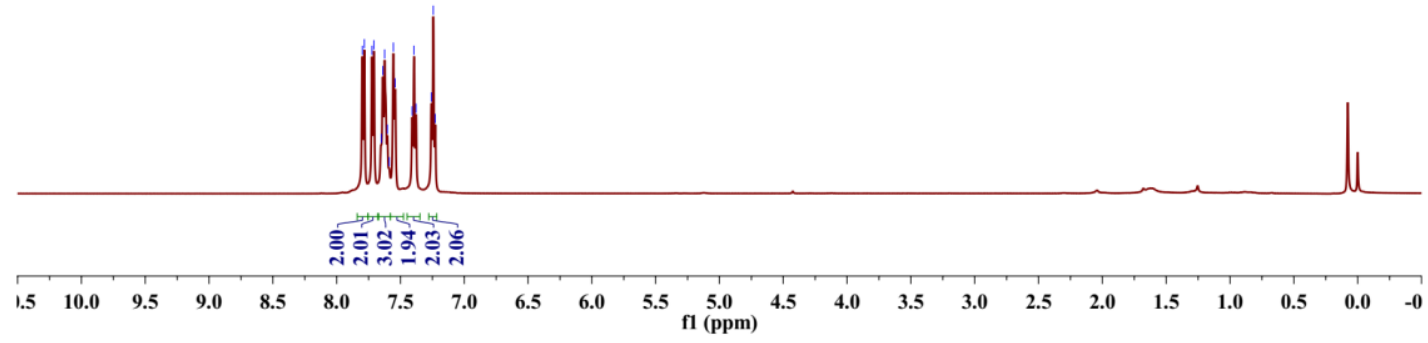

$>{ }^{13} \mathrm{C}$ NMR spectrum for $\mathbf{4 a}$

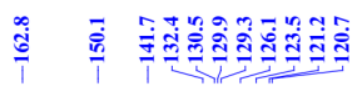

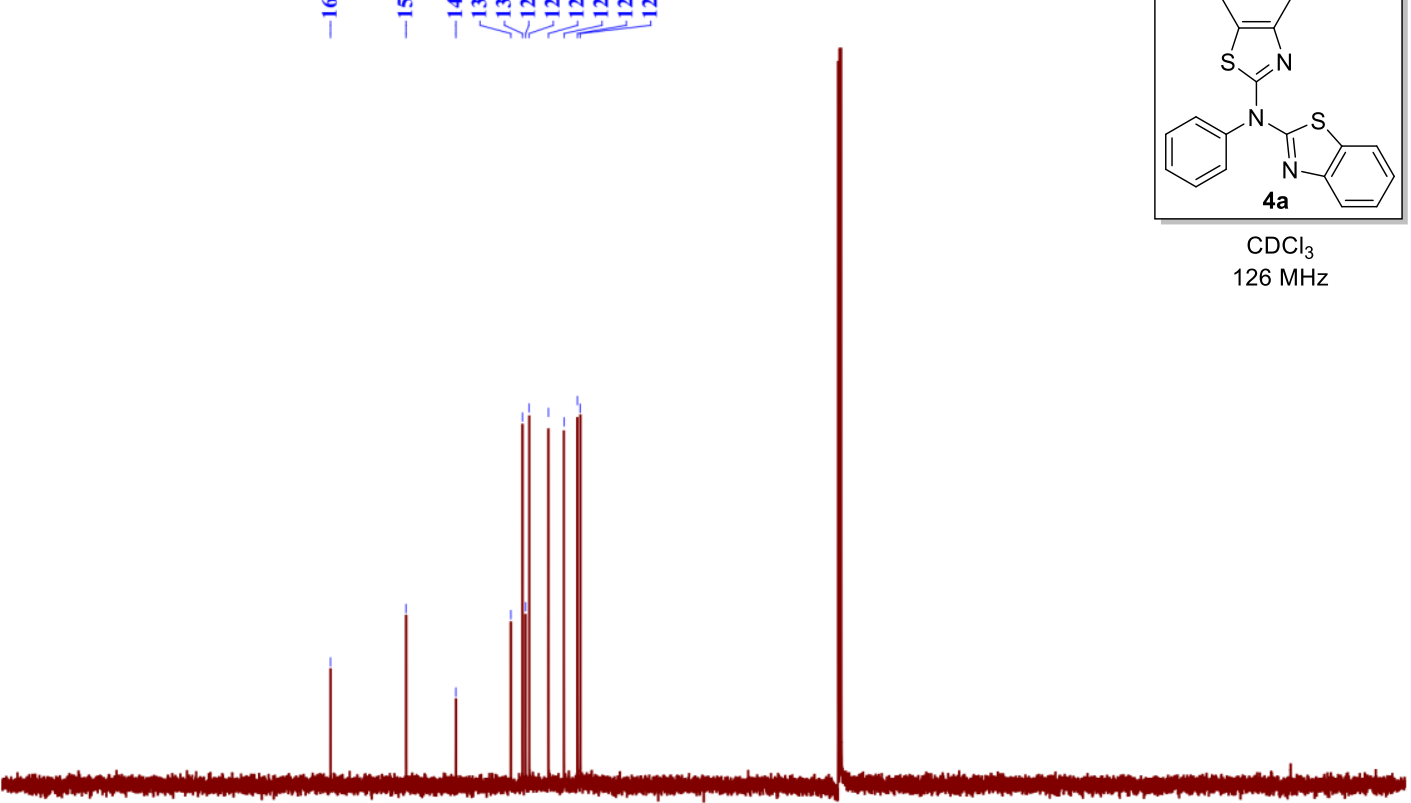

$\begin{array}{lllllllllllllllllllllll}210 & 200 & 190 & 180 & 170 & 160 & 150 & 140 & 130 & 120 & 110 & \begin{array}{c}100 \\ \mathrm{fl}(\mathrm{ppm})\end{array} & 90 & 80 & 70 & 60 & 50 & 40 & 30 & 20 & 10 & 0 & -10\end{array}$ 
${ }^{1} \mathrm{H}$ NMR spectrum for $\mathbf{4 b}$

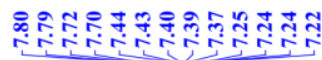

ฟิ

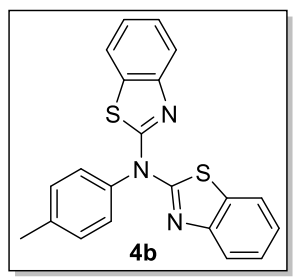

$\mathrm{CDCl}_{3}$ $500 \mathrm{MHz}$

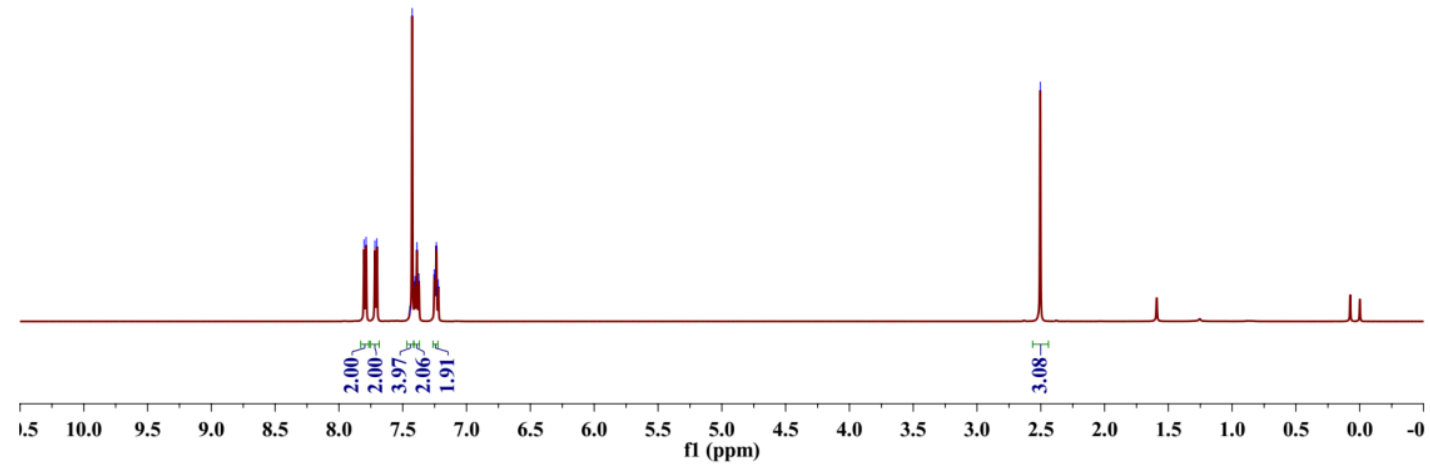

$>{ }^{13} \mathrm{C}$ NMR spectrum for $\mathbf{4 b}$
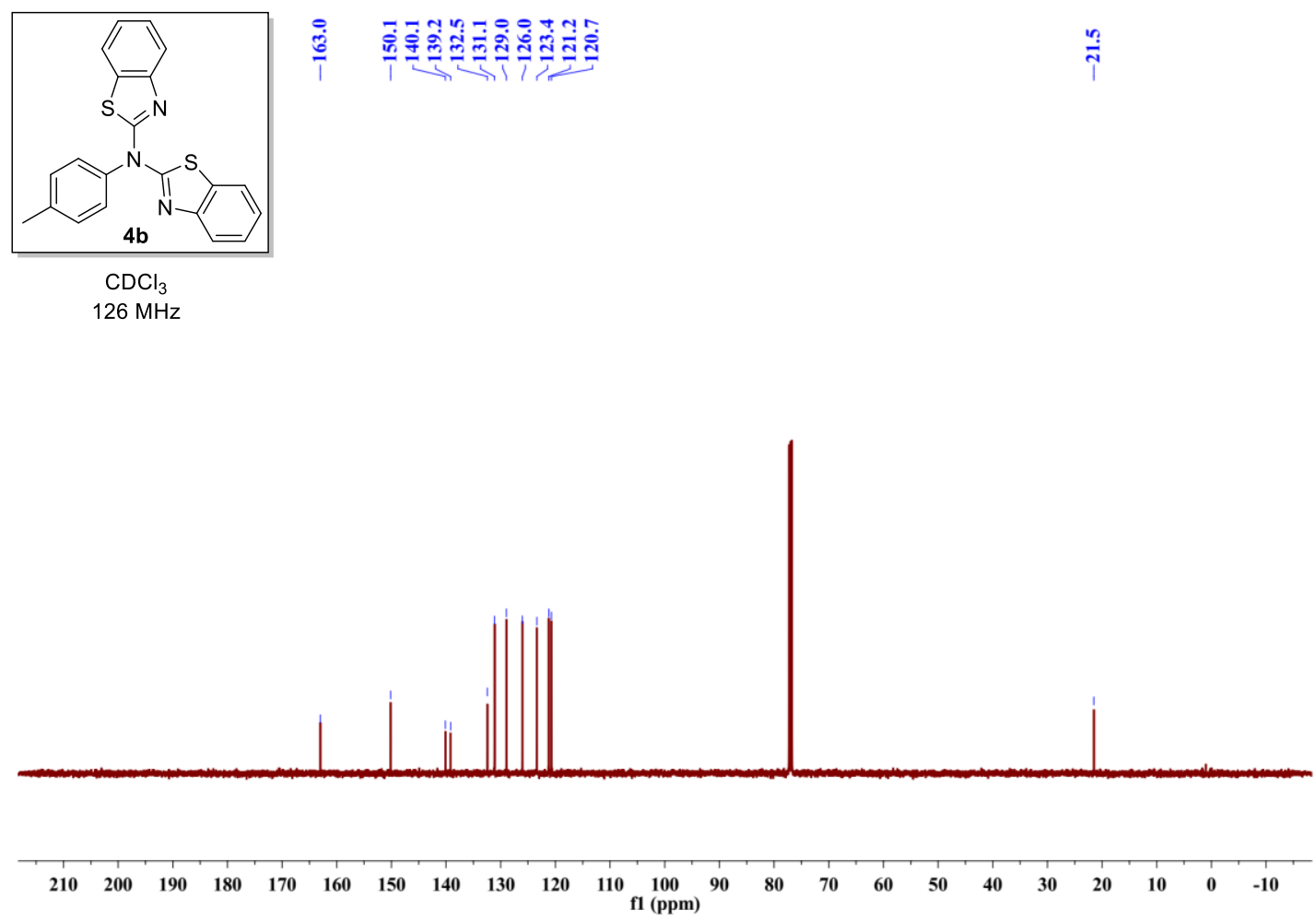
${ }^{1} \mathrm{H}$ NMR spectrum for $\mathbf{4 c}$

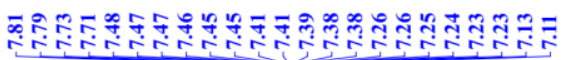

จี

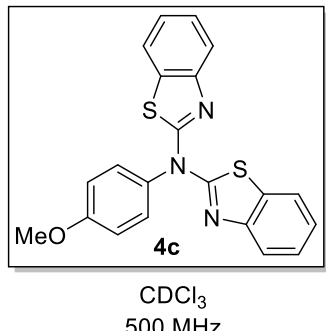

$500 \mathrm{MHz}$

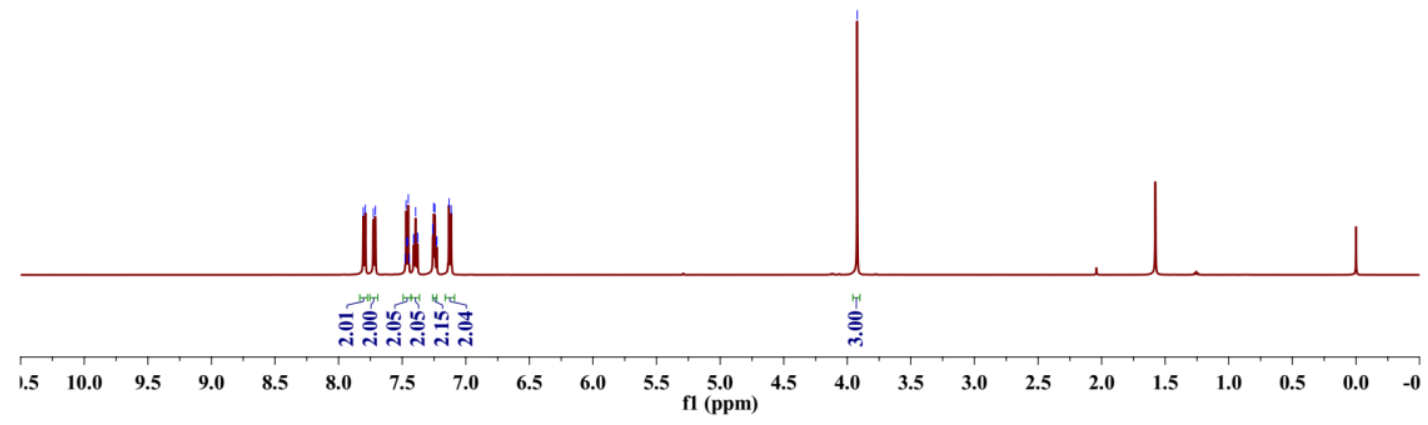

$>{ }^{13} \mathrm{C}$ NMR spectrum for $\mathbf{4 c}$

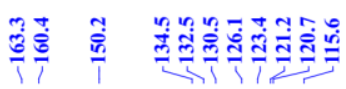

i.
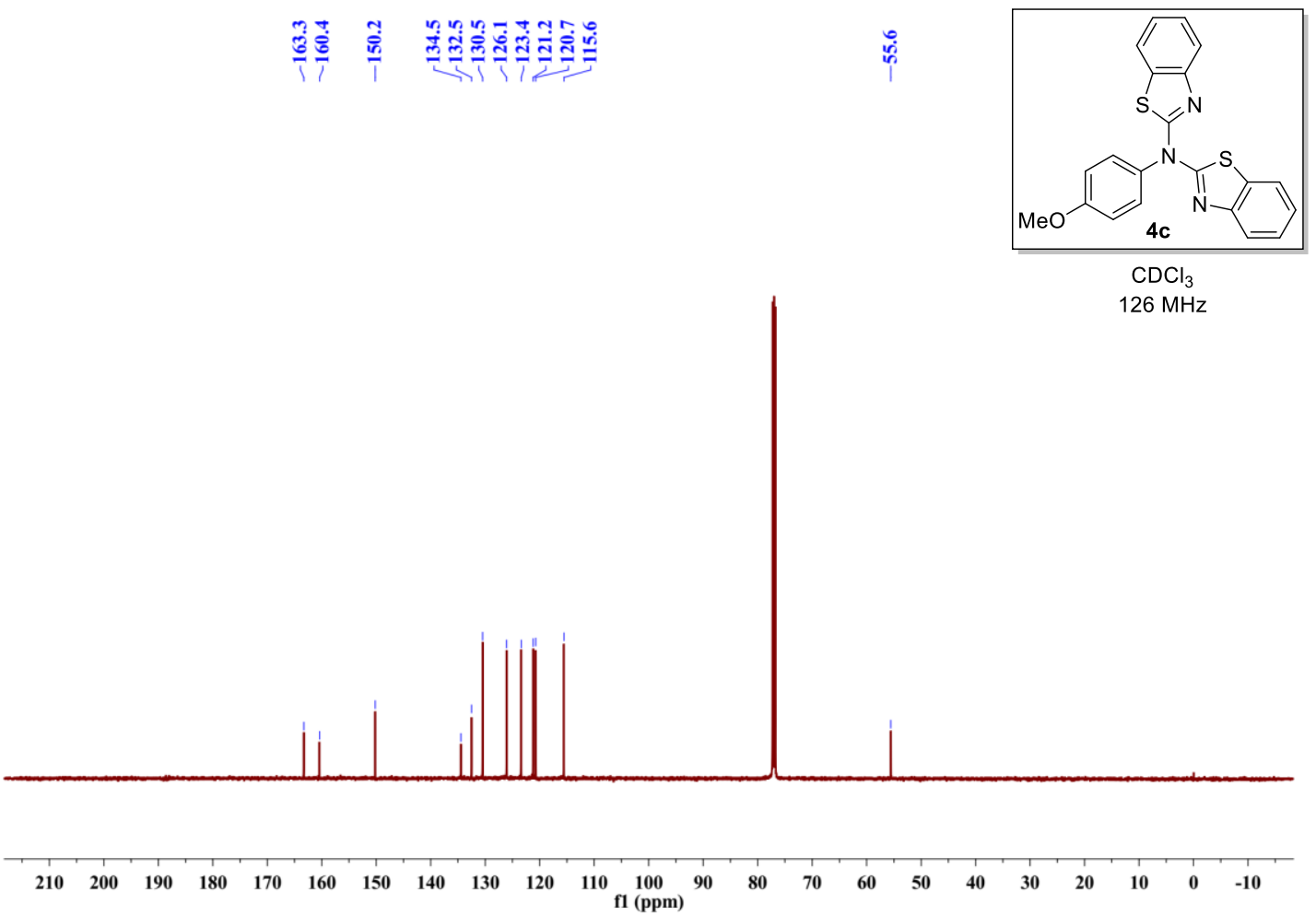
${ }^{1} \mathrm{H}$ NMR spectrum for $\mathbf{4 d}$

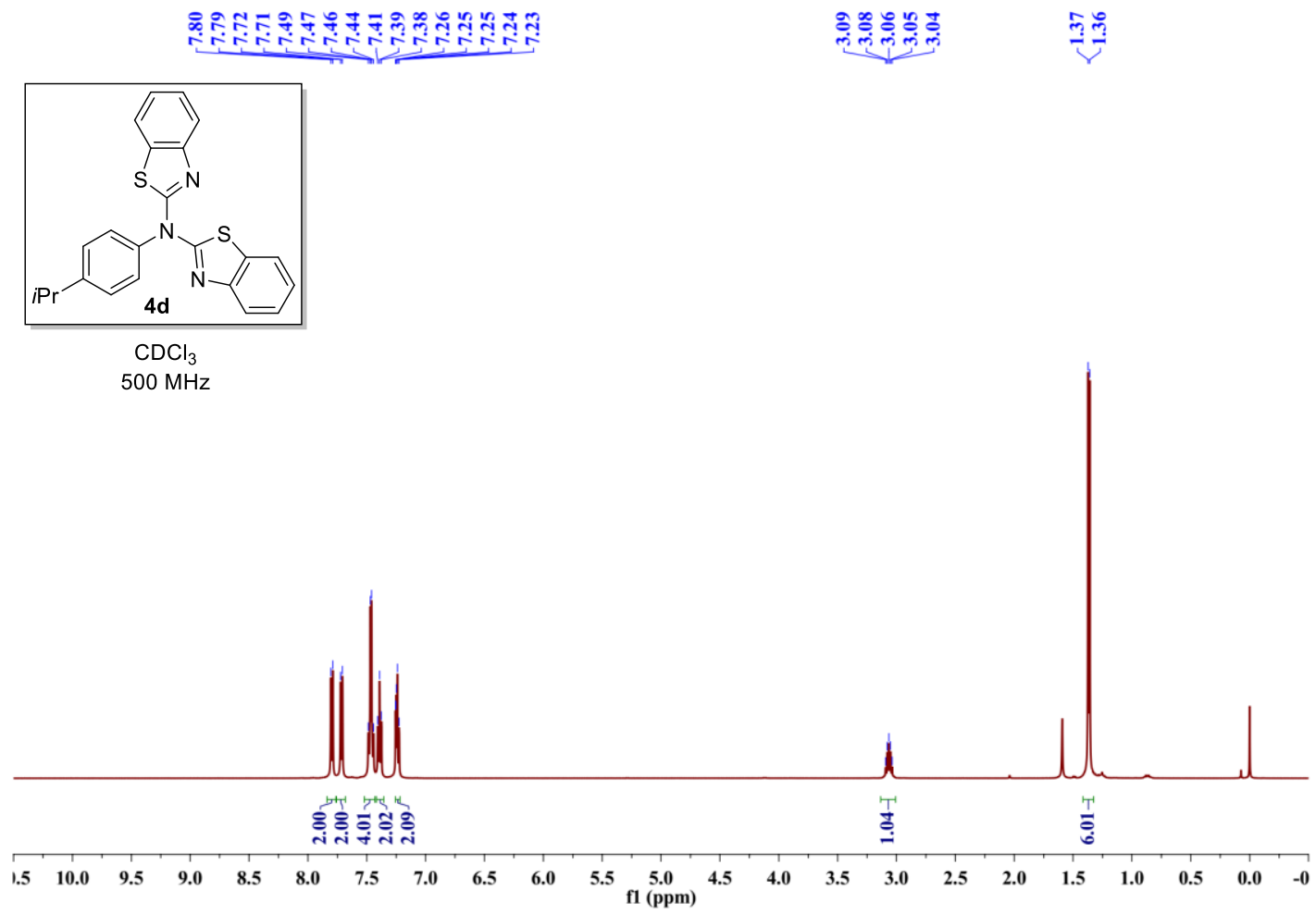

$>{ }^{13} \mathrm{C}$ NMR spectrum for $\mathbf{4 d}$

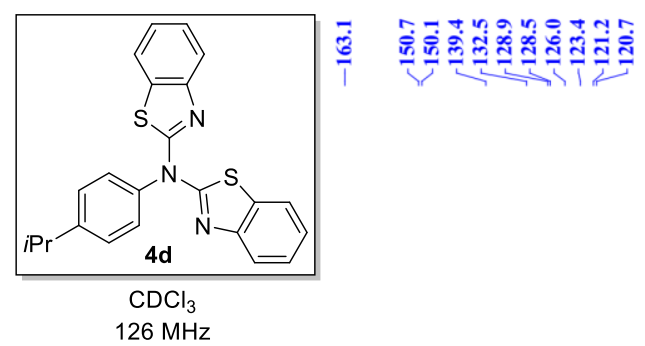

ثें సें

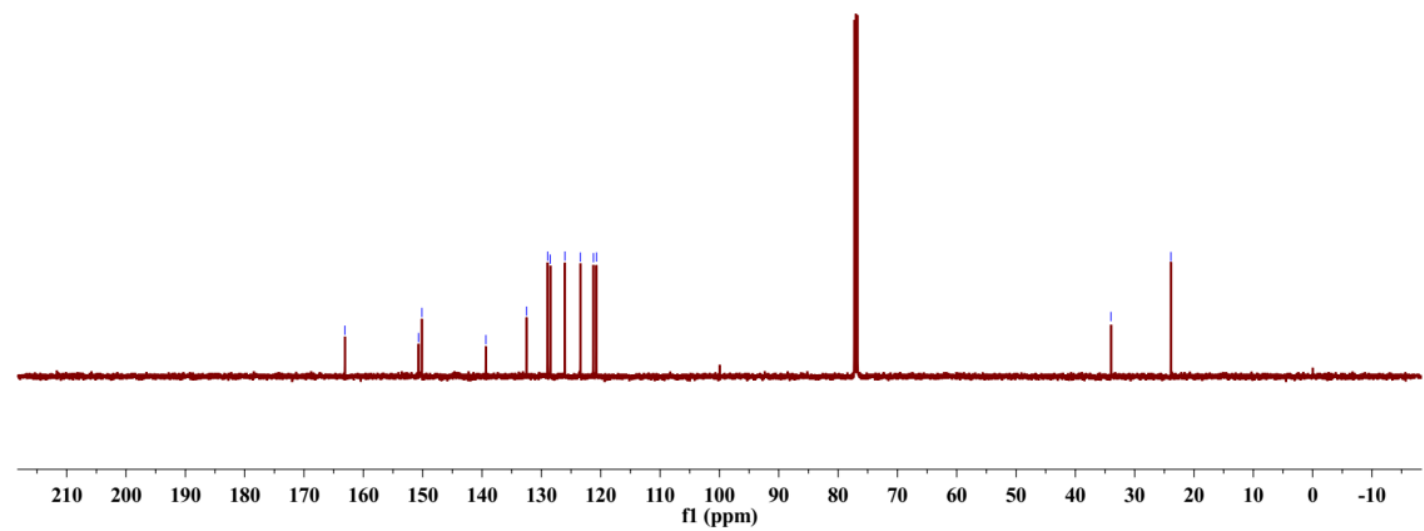


${ }^{1} \mathrm{H}$ NMR spectrum for $\mathbf{4 e}$

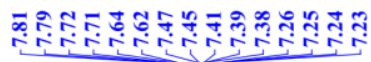

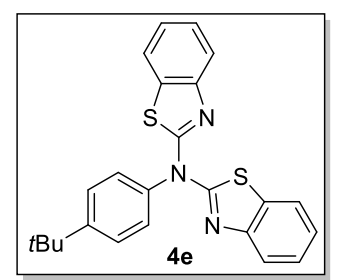

$\mathrm{CDCl}_{3}$

$500 \mathrm{MHz}$

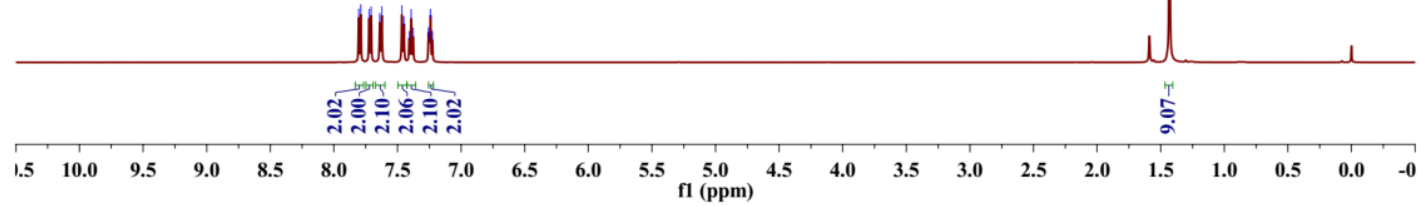

${ }^{13} \mathrm{C}$ NMR spectrum for $4 \mathrm{e}$
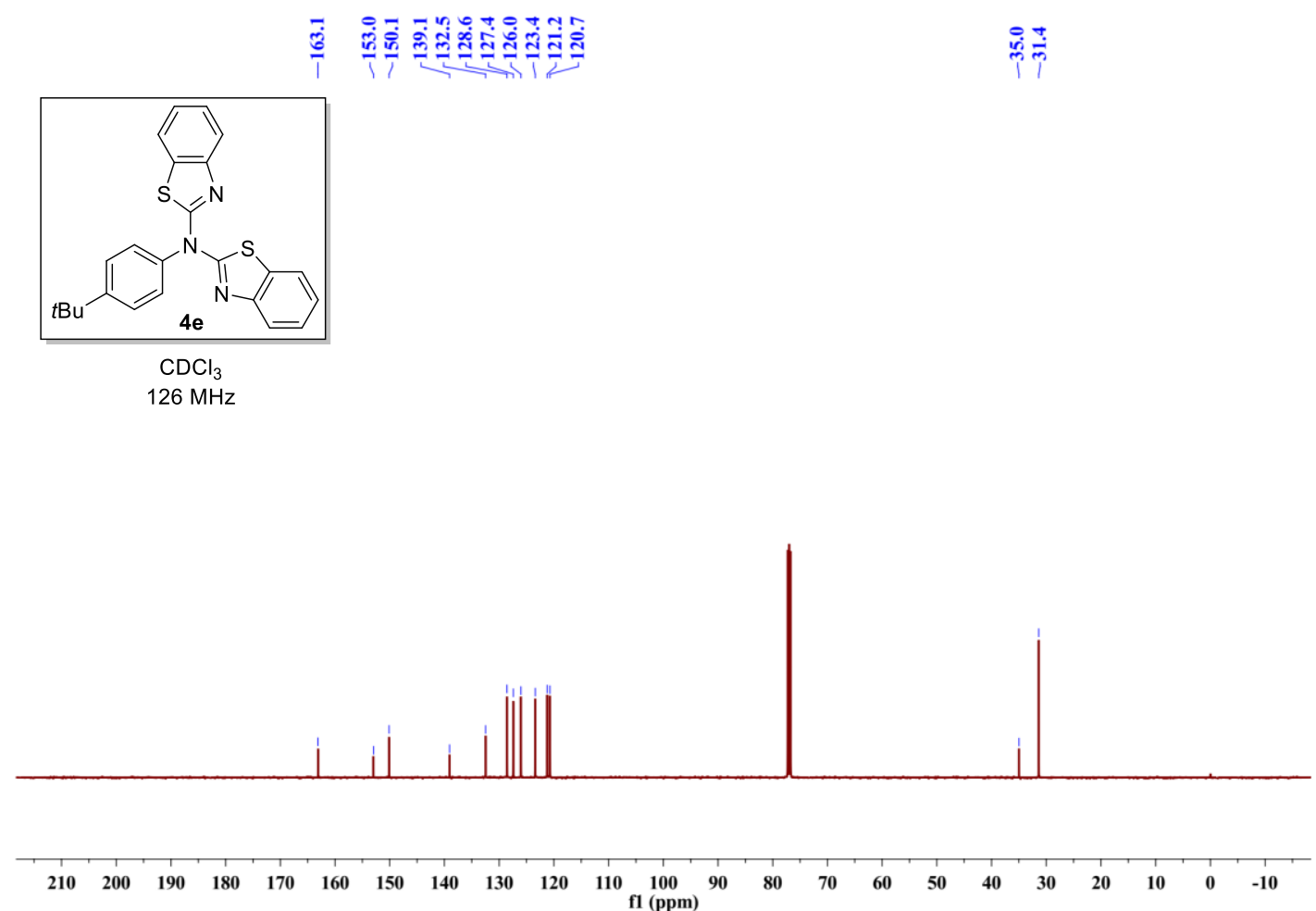
${ }^{1} \mathrm{H}$ NMR spectrum for $\mathbf{4 f}$

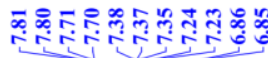

$\stackrel{5}{\circ}$
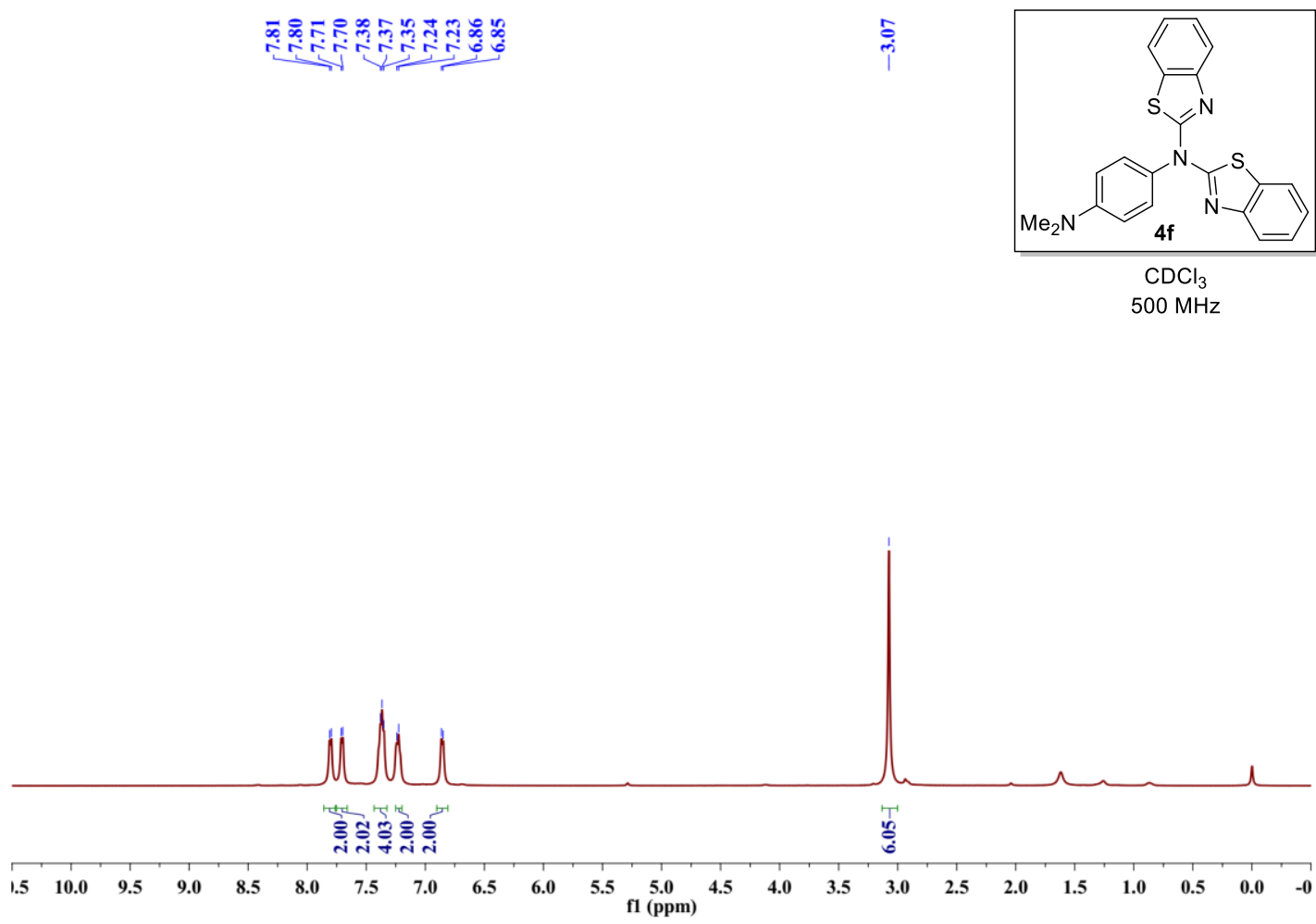

$>{ }^{13} \mathrm{C}$ NMR spectrum for $\mathbf{4 f}$
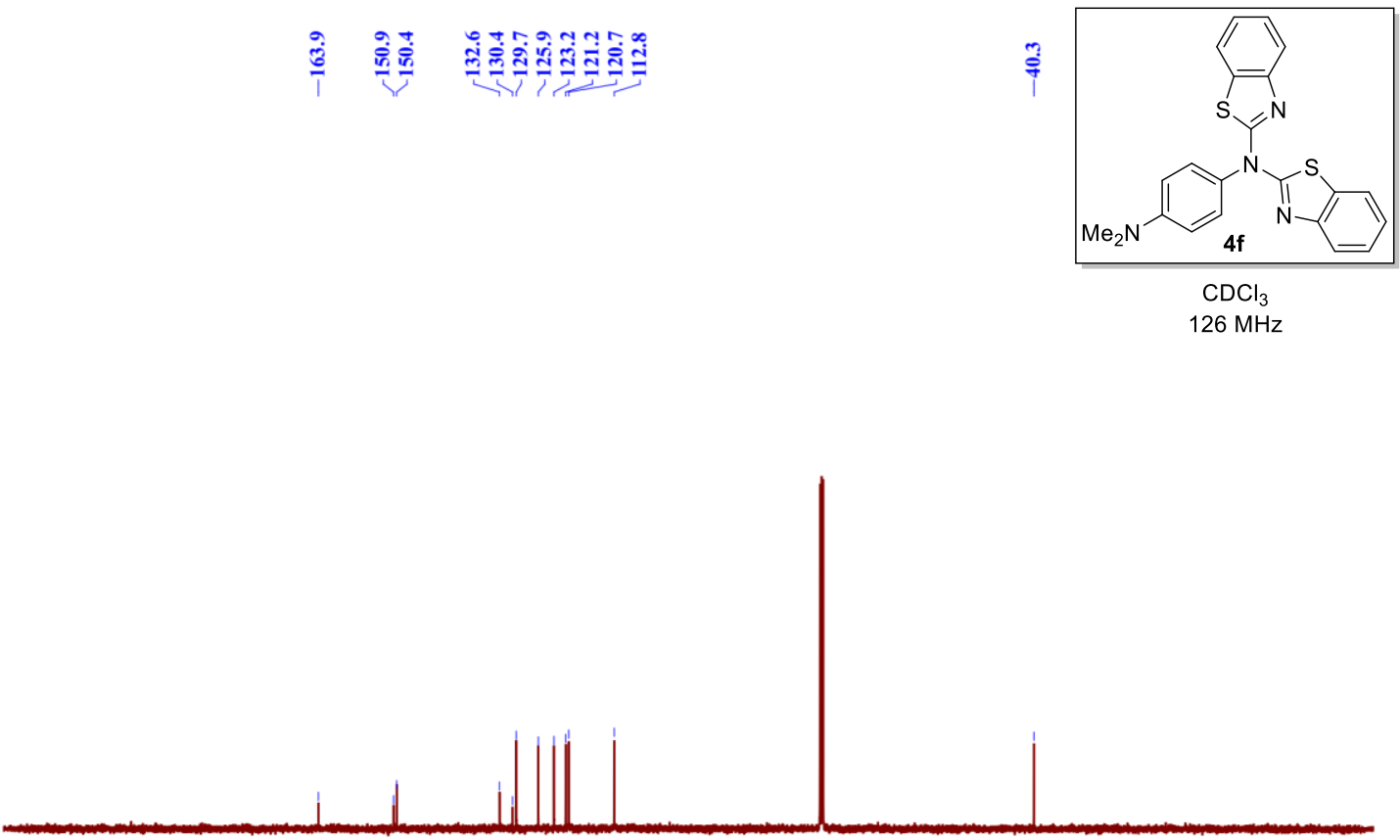

$\begin{array}{lllllllllllllllllllllll}210 & 200 & 190 & 180 & 170 & 160 & 150 & 140 & 130 & 120 & 110 & \begin{array}{c}100 \\ 10\end{array} & 90 & 80 & 70 & 60 & 50 & 40 & 30 & 20 & 10 & 0 & -10\end{array}$ 
${ }^{1} \mathrm{H}$ NMR spectrum for $\mathbf{4 g}$

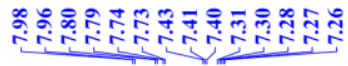

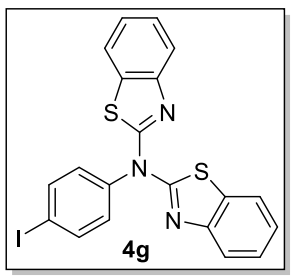

$\mathrm{CDCl}_{3}$

$500 \mathrm{MHz}$

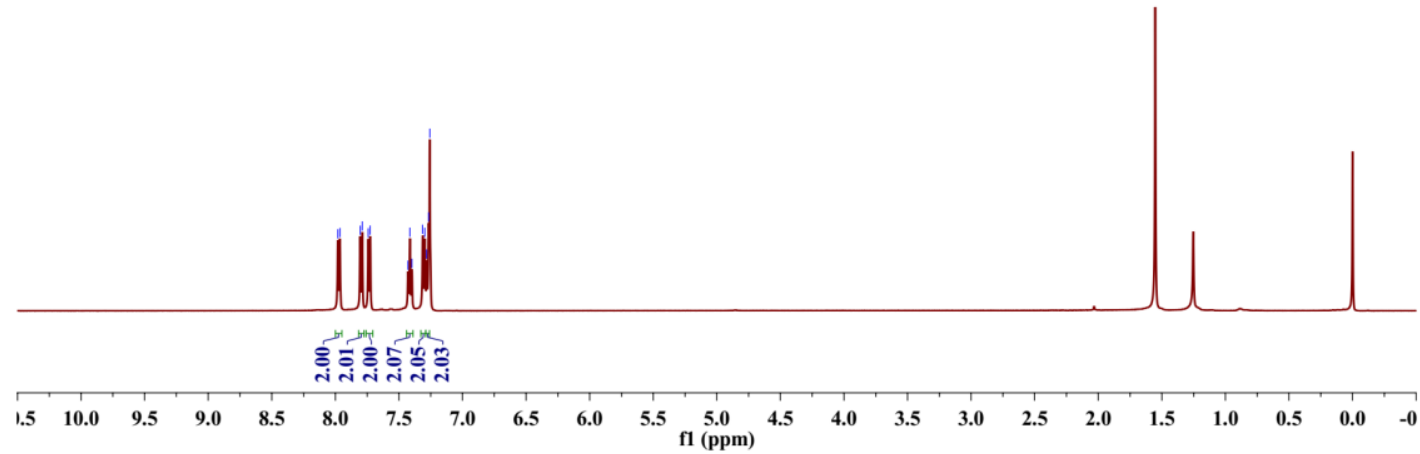

$>{ }^{13} \mathrm{C}$ NMR spectrum for $\mathbf{4 g}$

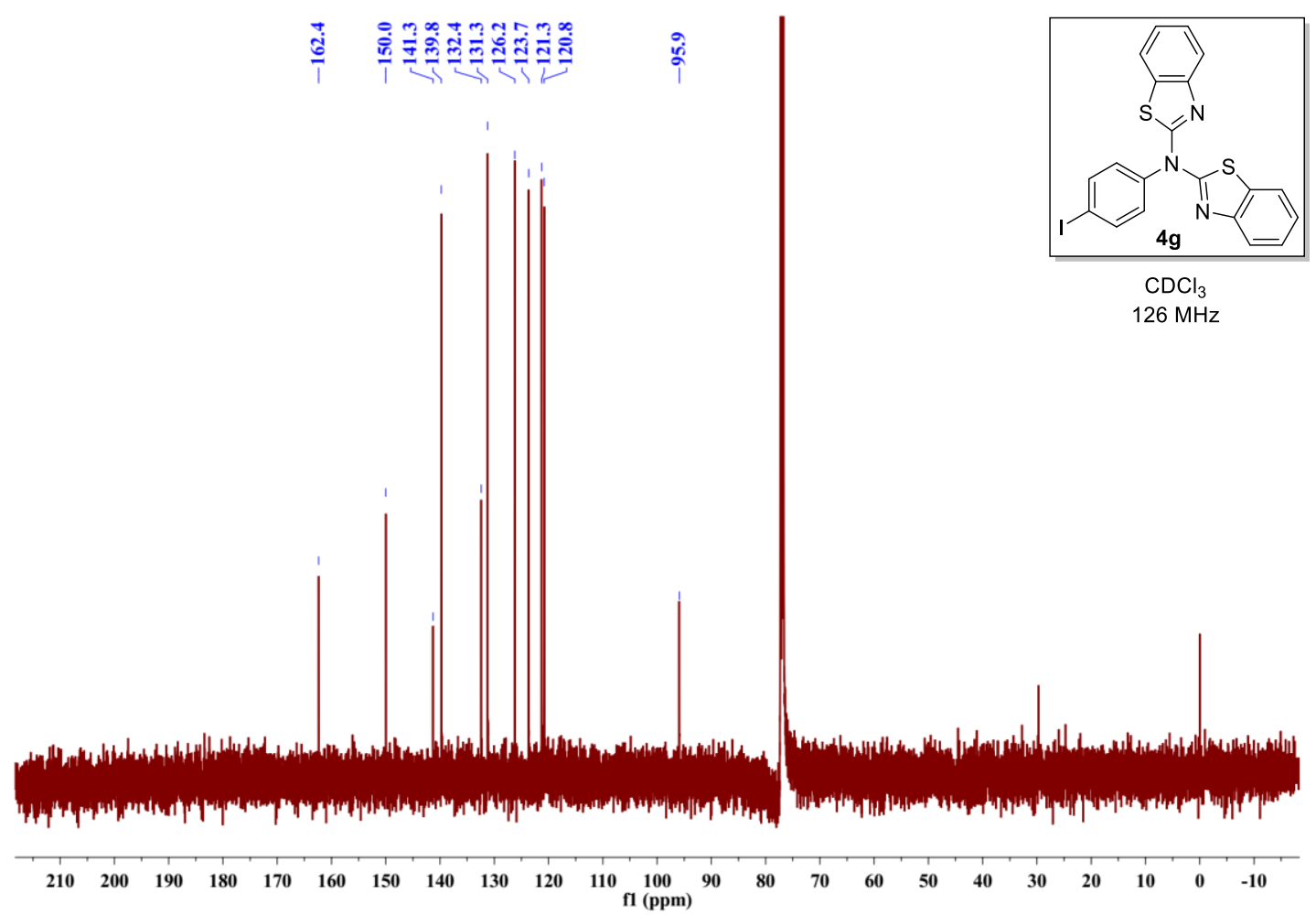


${ }^{1} \mathrm{H}$ NMR spectrum for $\mathbf{4 h}$

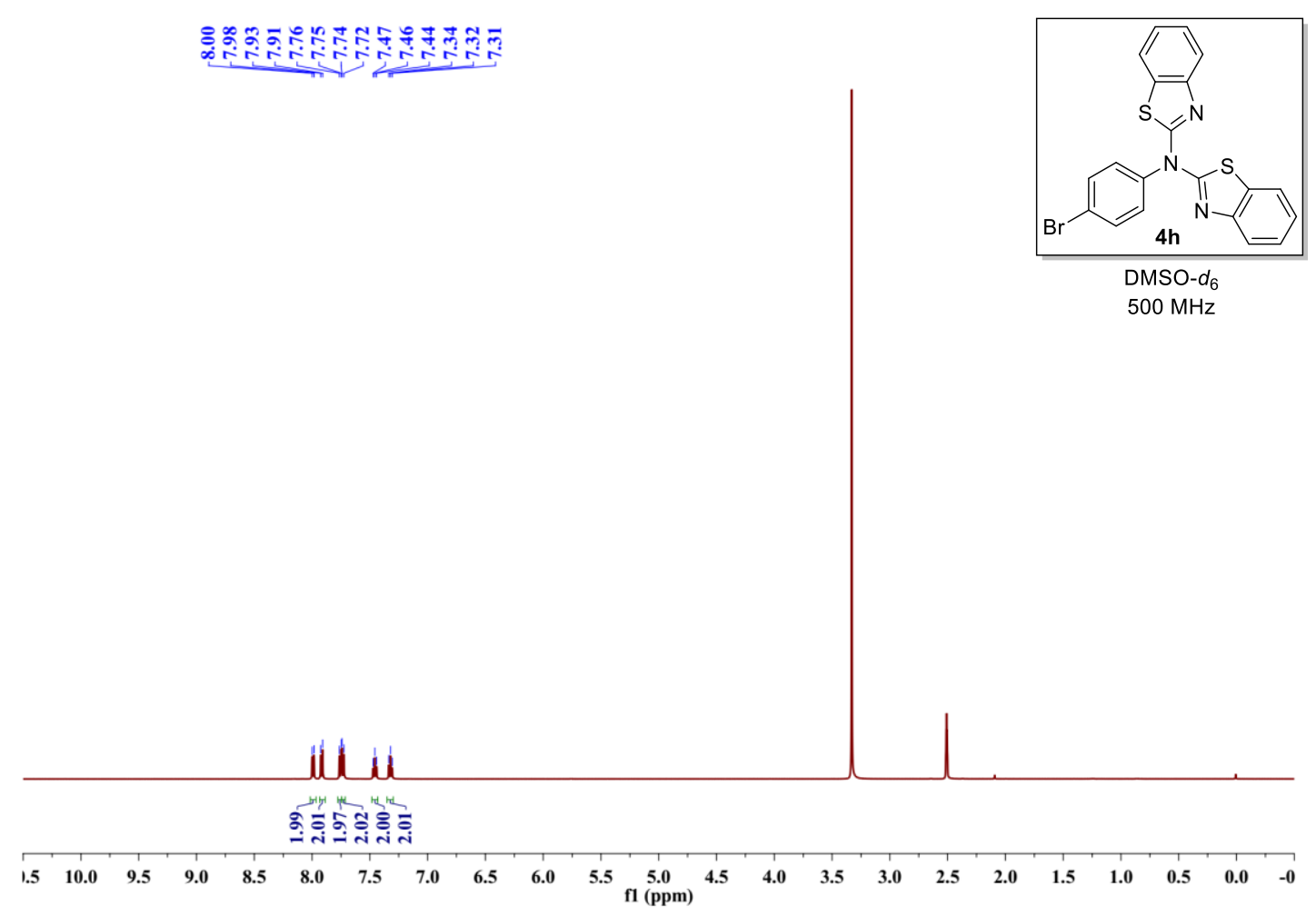

$>{ }^{13} \mathrm{C}$ NMR spectrum for $\mathbf{4 h}$

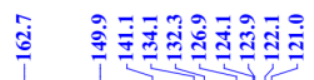

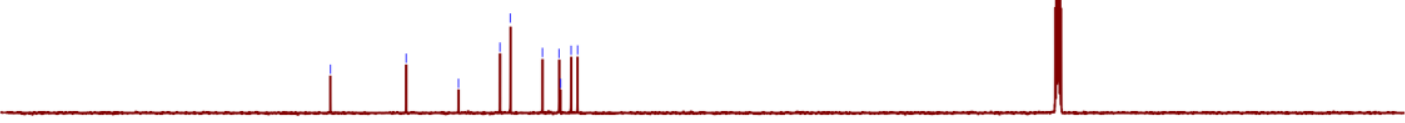

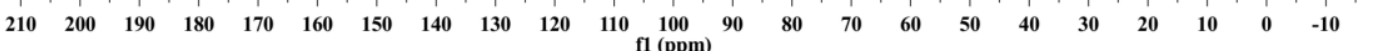


${ }^{1} \mathrm{H}$ NMR spectrum for $\mathbf{4 i}$

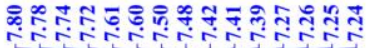
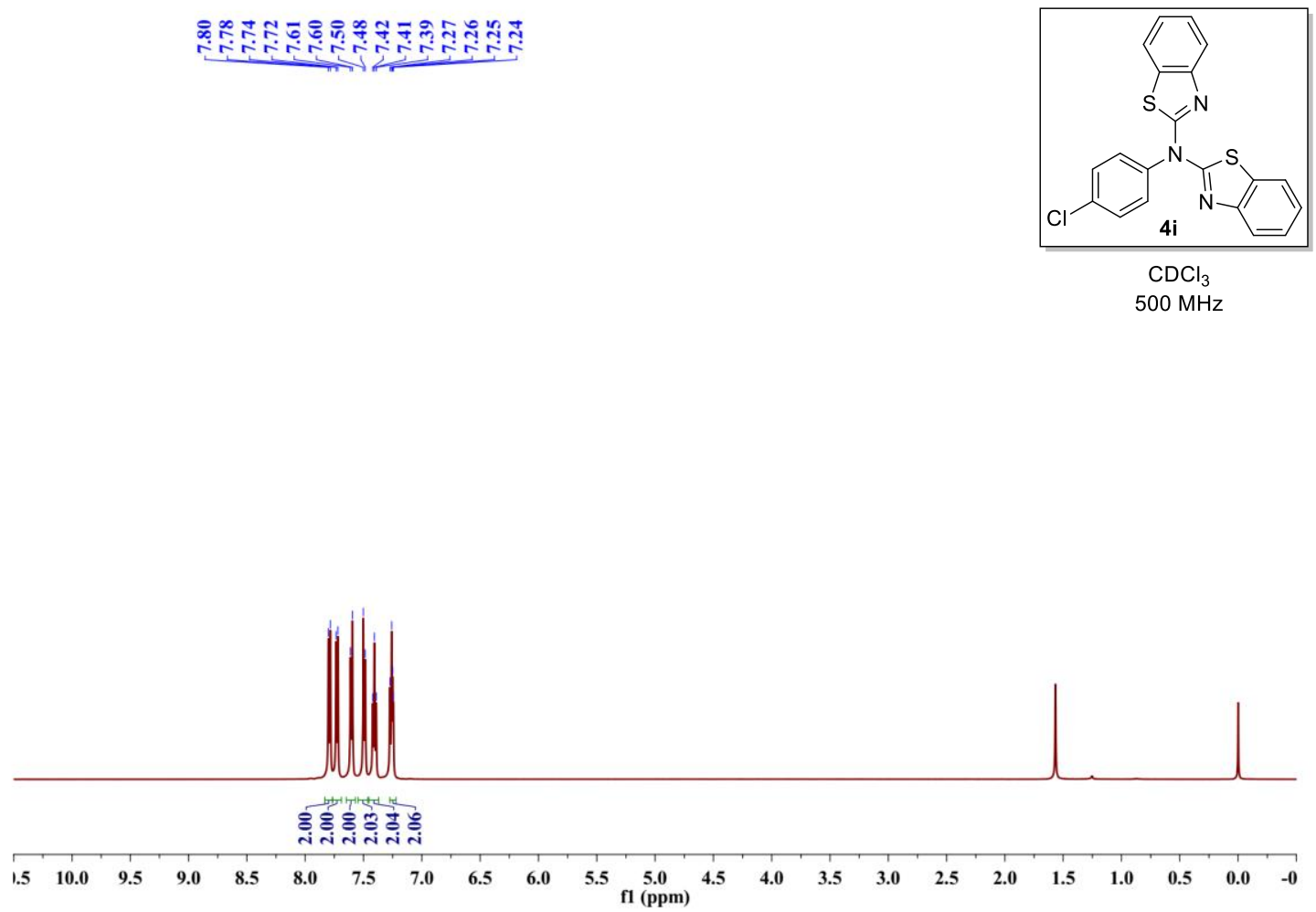

$>{ }^{13} \mathrm{C}$ NMR spectrum for $\mathbf{4 i}$

菅
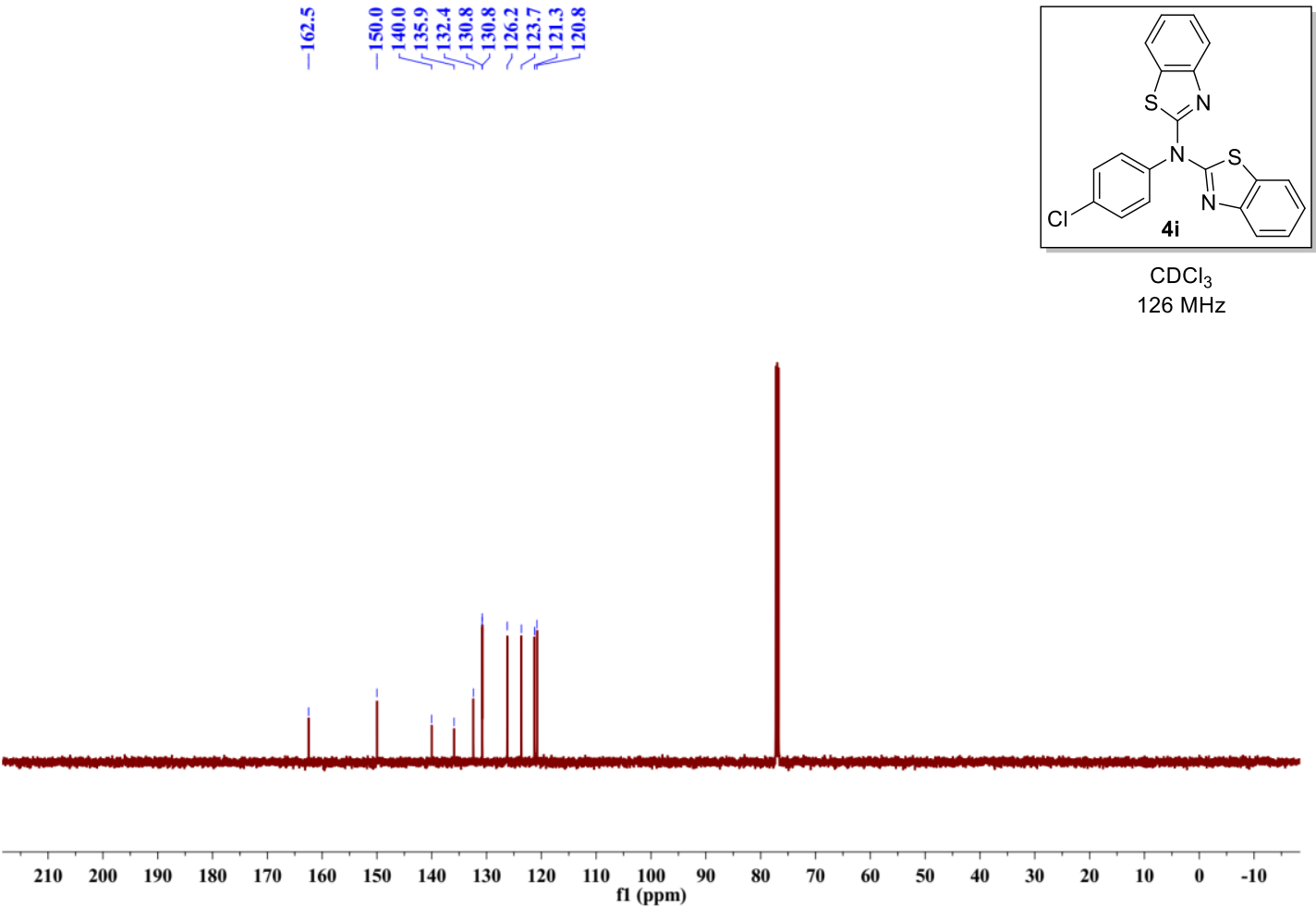
${ }^{1} \mathrm{H}$ NMR spectrum for $\mathbf{4 j}$

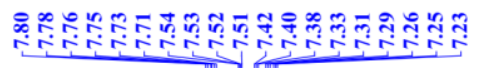
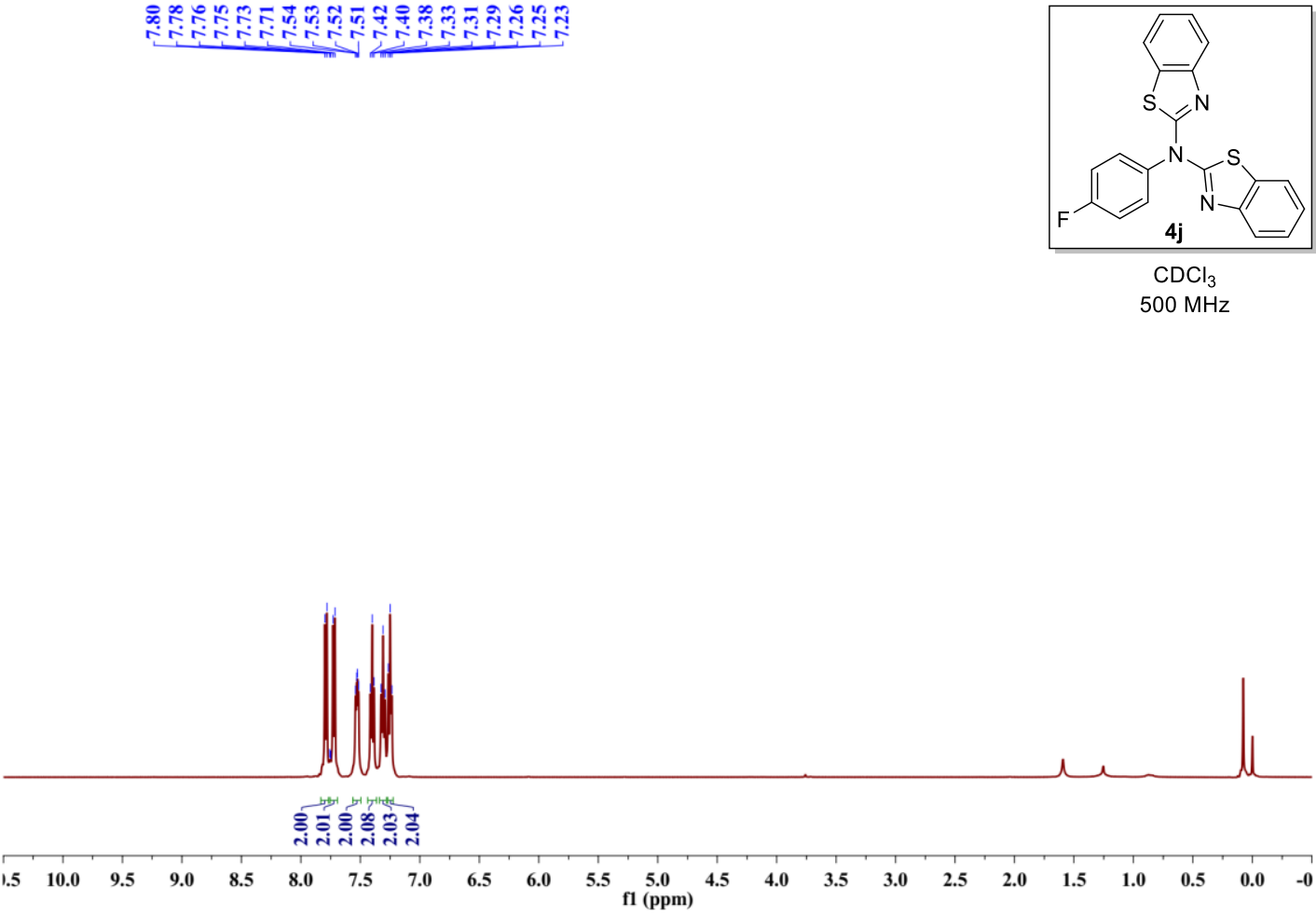

$>{ }^{13} \mathrm{C}$ NMR spectrum for $\mathbf{4 j}$

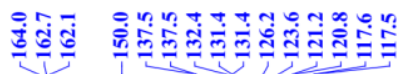
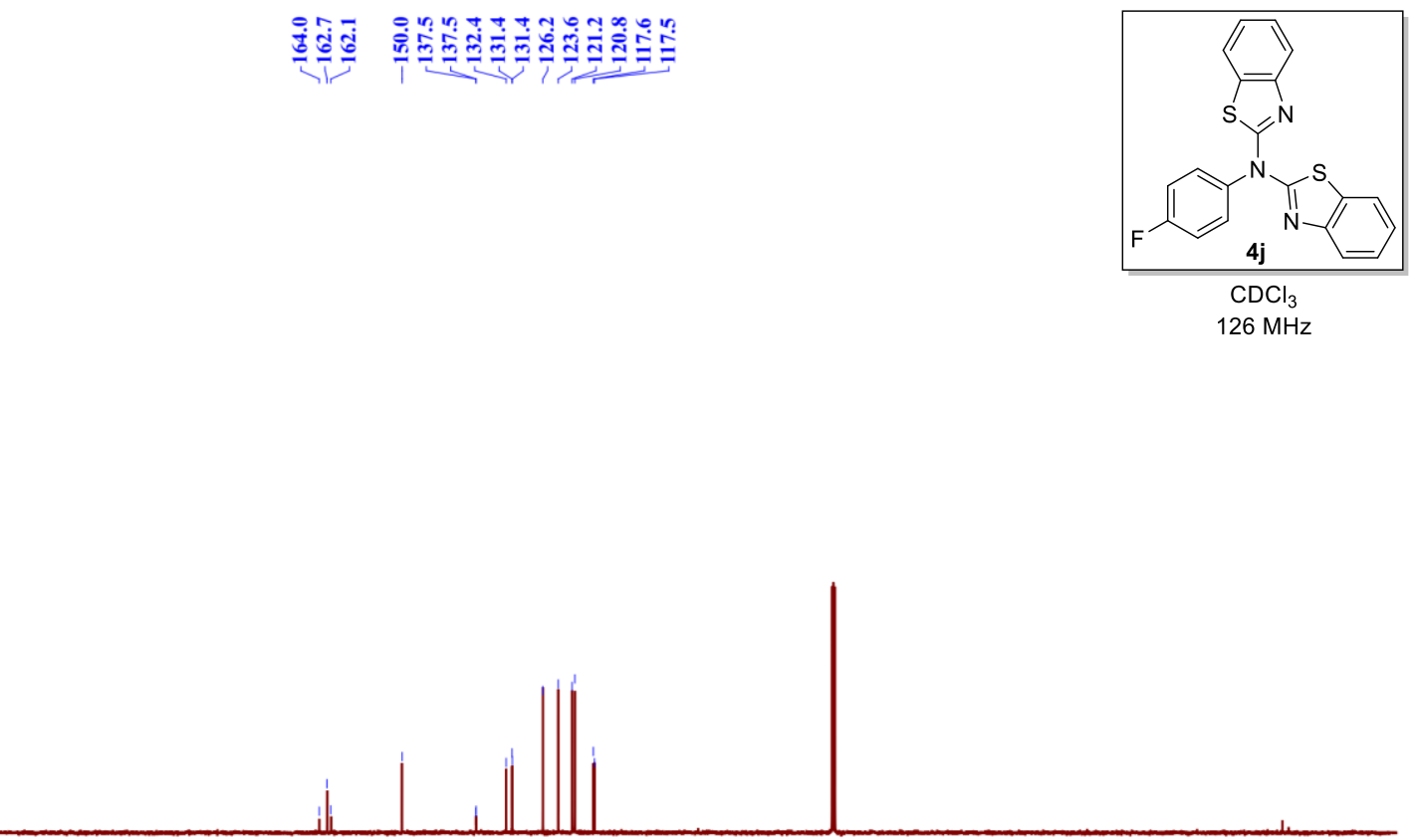

$\begin{array}{lllllllllllllllllllllll}210 & 200 & 190 & 180 & 170 & 160 & 150 & 140 & 130 & 120 & 110 & 100 & 90 & 80 & 70 & 60 & 50 & 40 & 30 & 20 & 10 & 0 & -10\end{array}$ 
${ }^{19} \mathrm{~F}$ NMR spectrum for $\mathbf{4 j}$

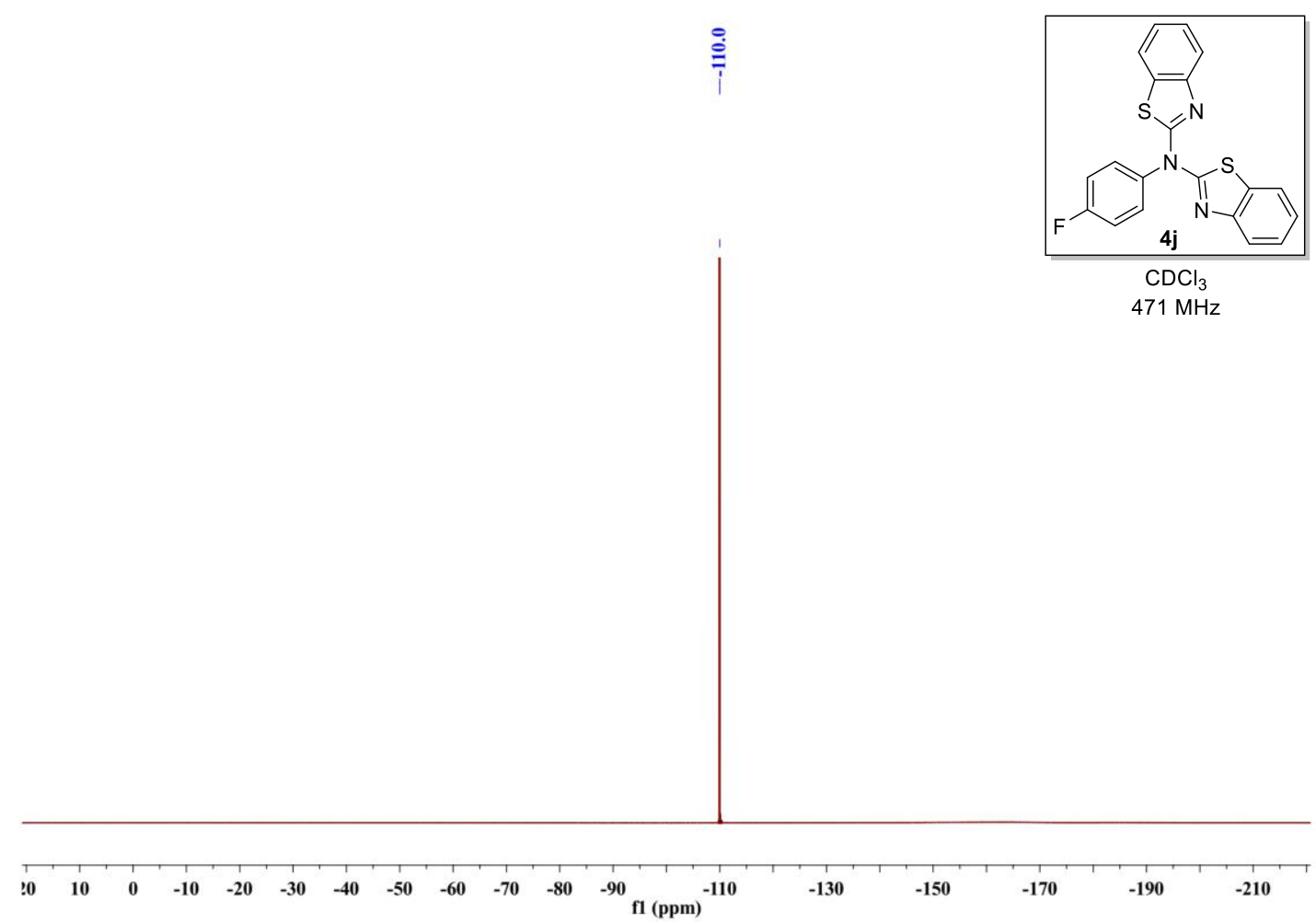


${ }^{1} \mathrm{H}$ NMR spectrum for $\mathbf{4 k}$

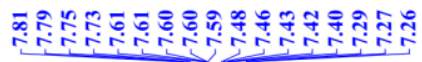
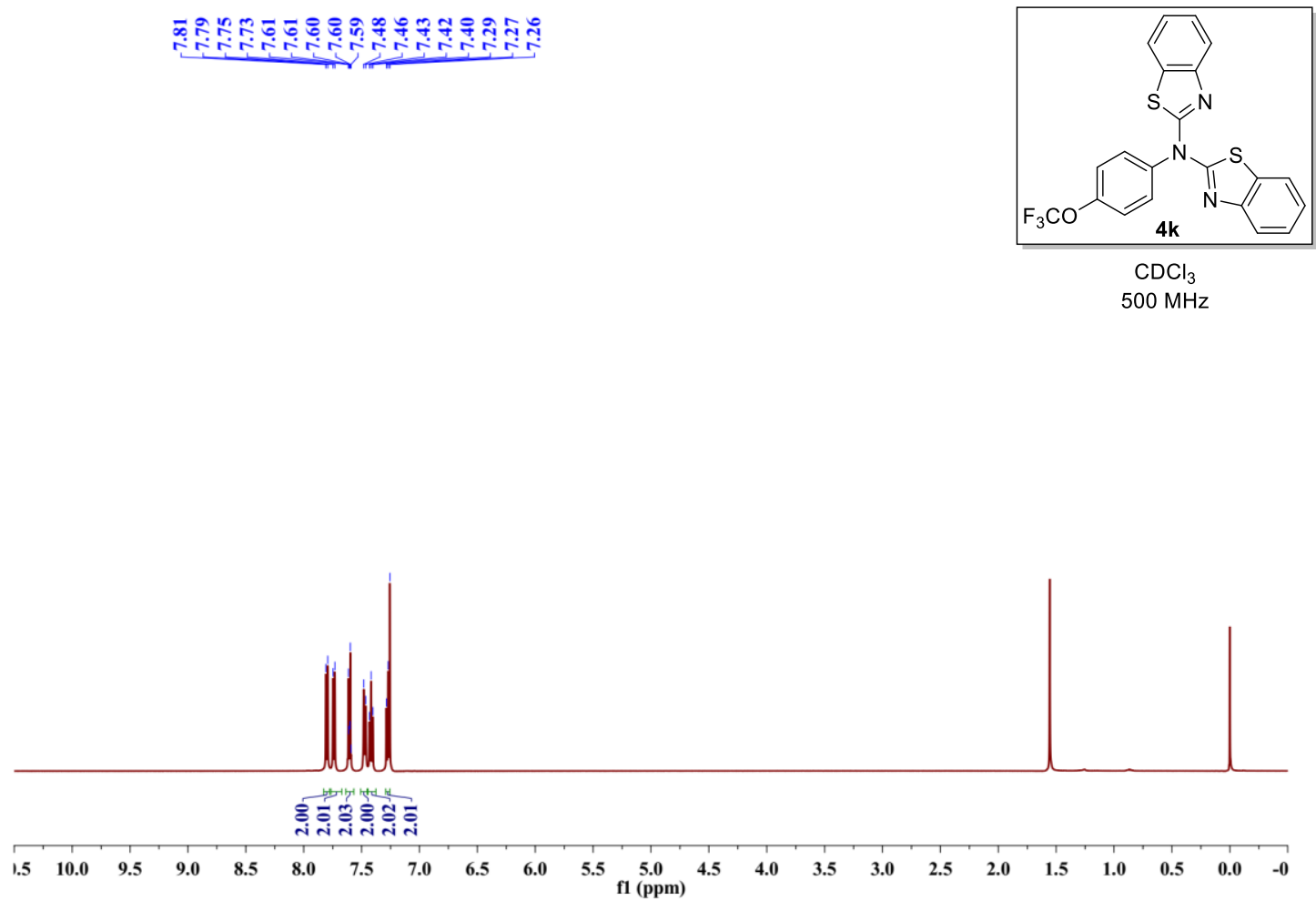

$>{ }^{13} \mathrm{C}$ NMR spectrum for $\mathbf{4 k}$

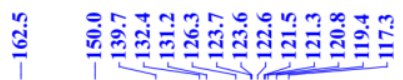

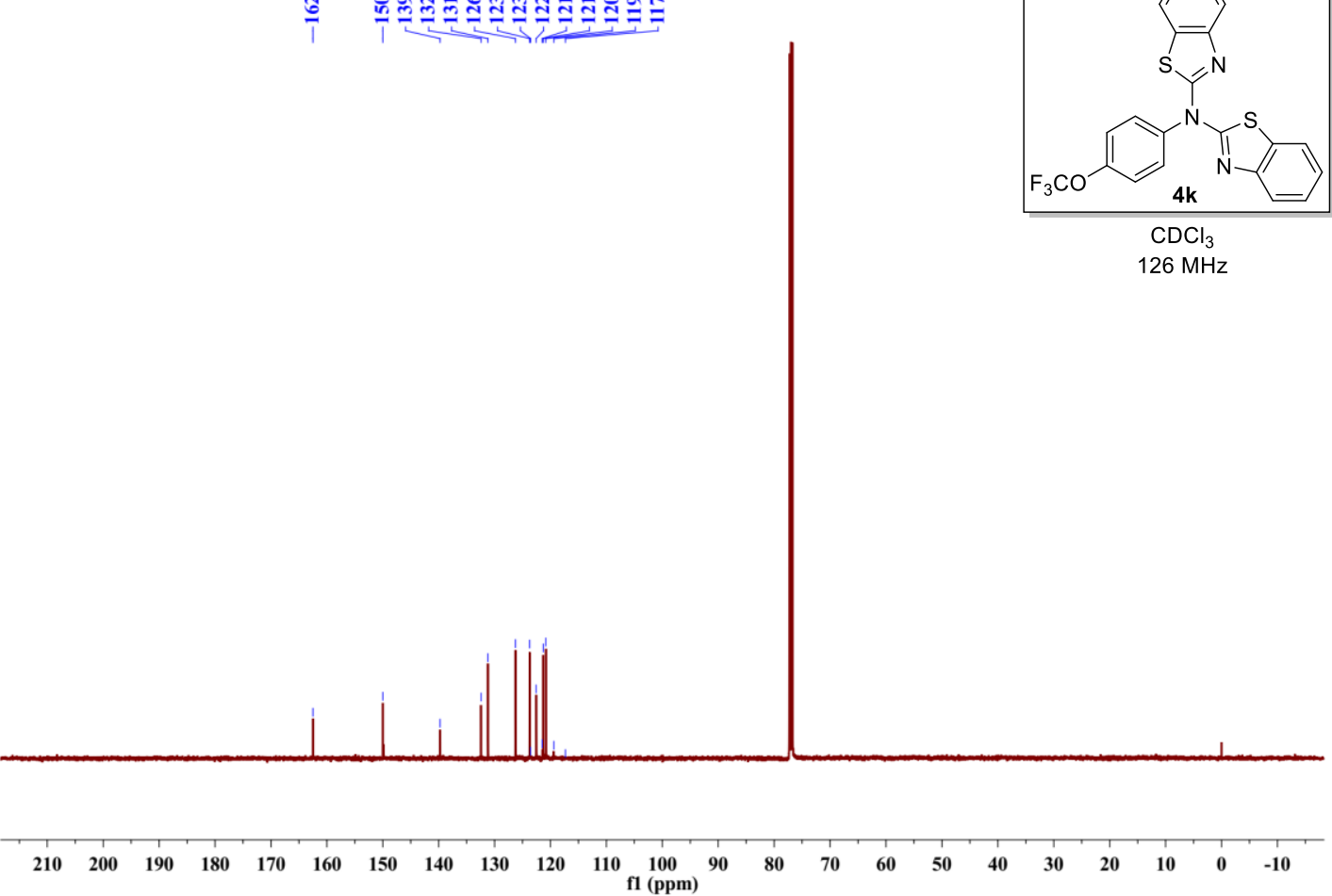


${ }^{19} \mathrm{~F}$ NMR spectrum for $\mathbf{4 k}$

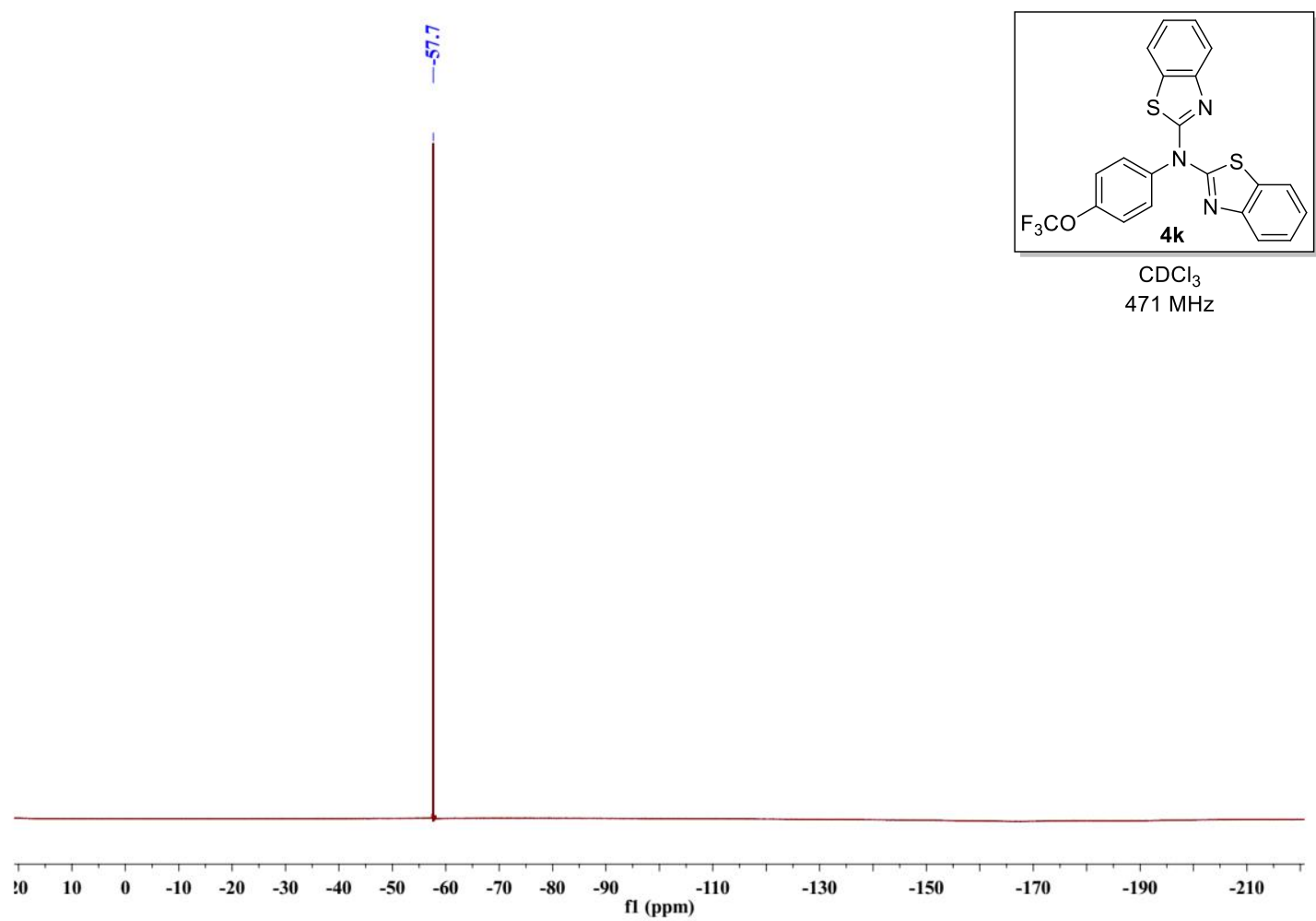


${ }^{1} \mathrm{H}$ NMR spectrum for $\mathbf{4 l}$

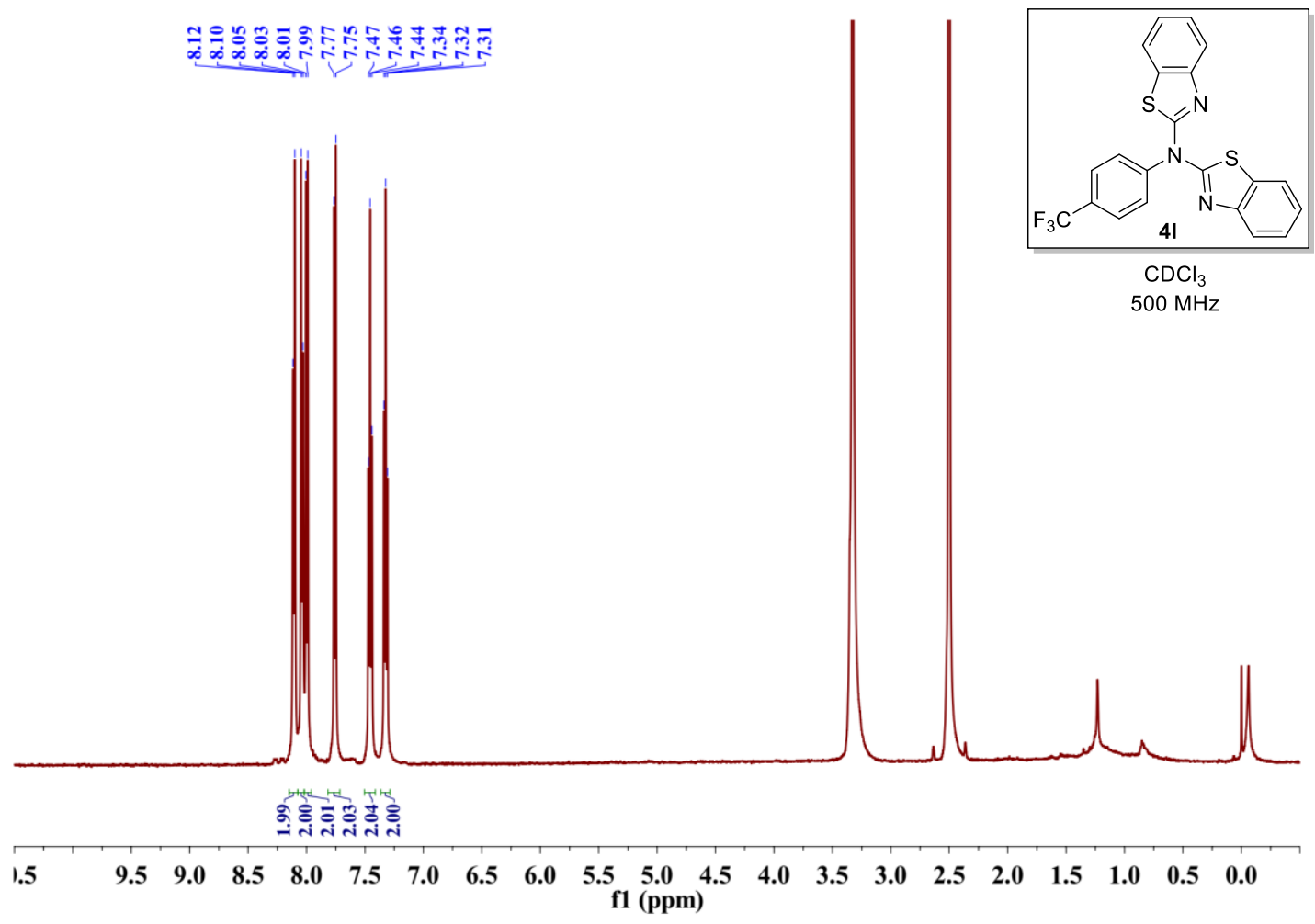

$>{ }^{13} \mathrm{C}$ NMR spectrum for $\mathbf{4 l}$

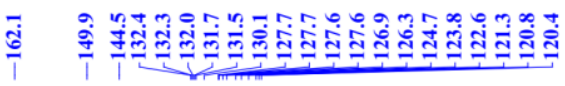

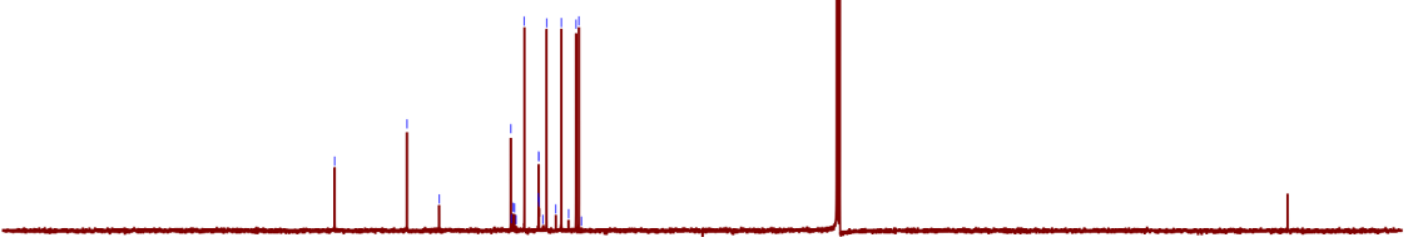

$\begin{array}{lllllllllllllllllllllll}210 & 200 & 190 & 180 & 170 & 160 & 150 & 140 & 130 & 120 & 110 & \begin{array}{c}100 \\ 100\end{array} & 90 & 70 & 60 & 50 & 40 & 30 & 20 & 10 & 0 & -10\end{array}$ 
${ }^{19}$ F NMR spectrum for $\mathbf{4 l}$

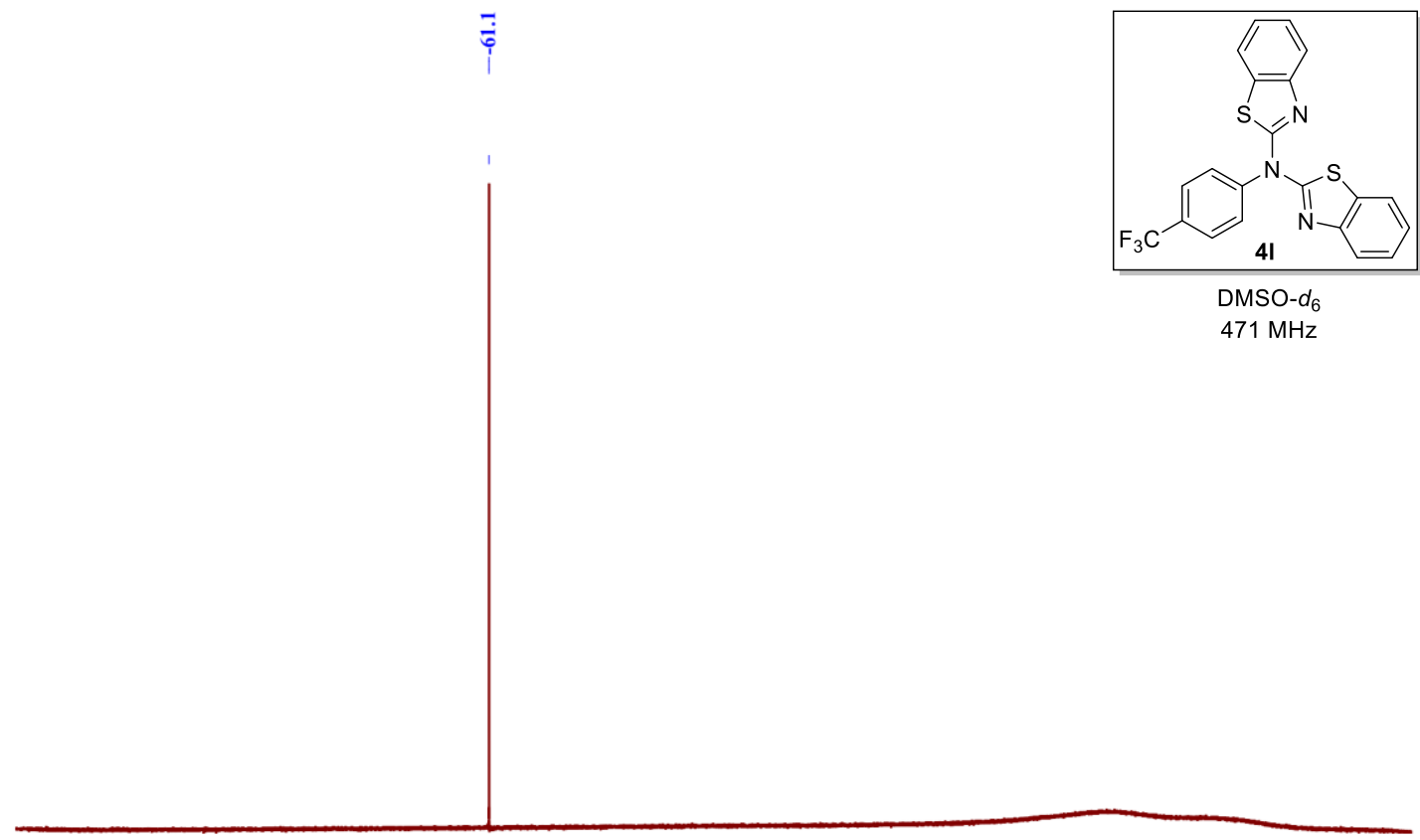

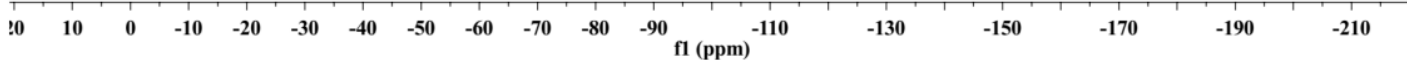


${ }^{1} \mathrm{H}$ NMR spectrum for $\mathbf{4 m}$

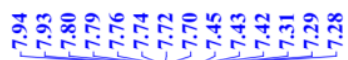
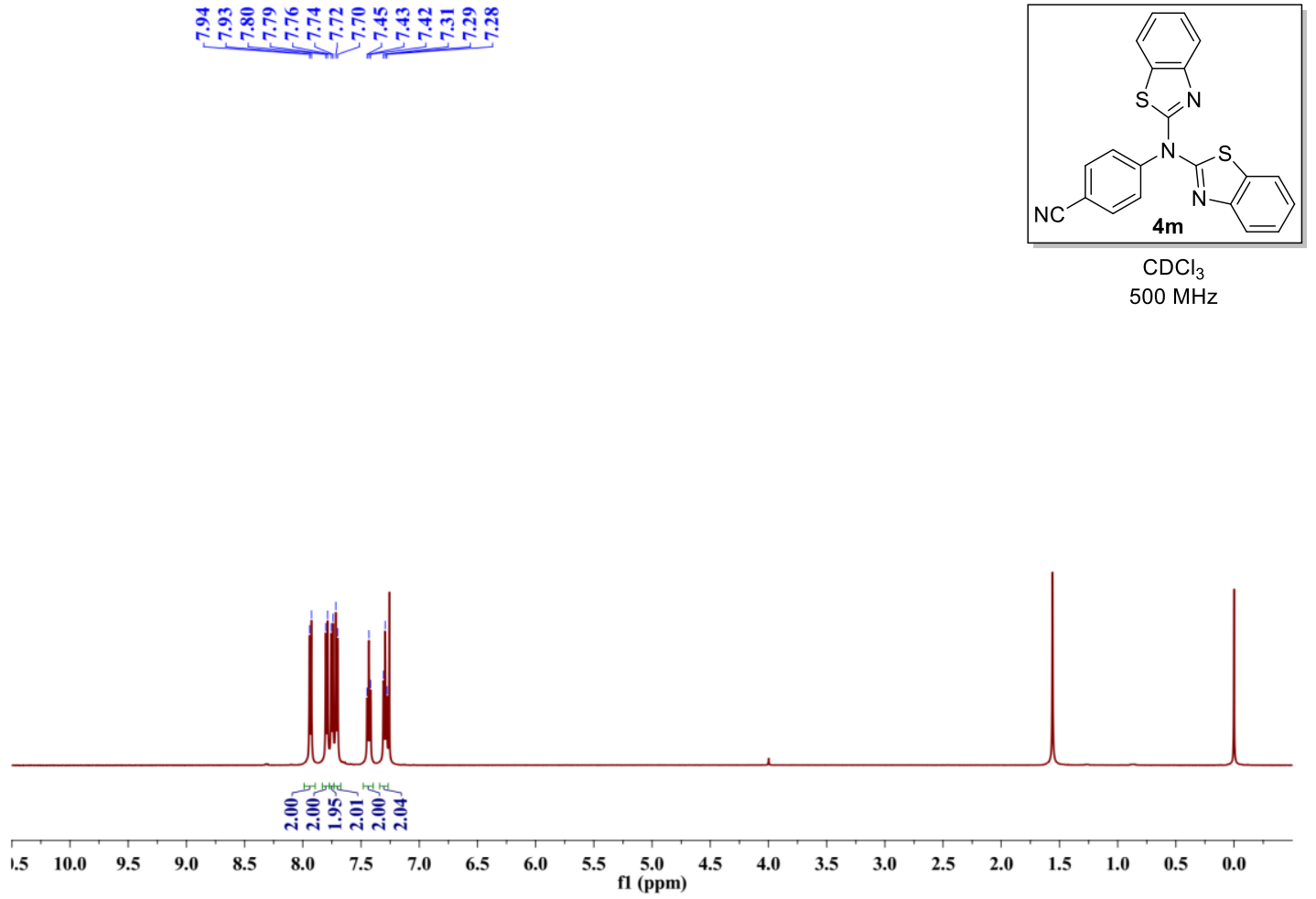

$>{ }^{13} \mathrm{C}$ NMR spectrum for $\mathbf{4 m}$

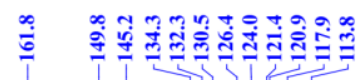

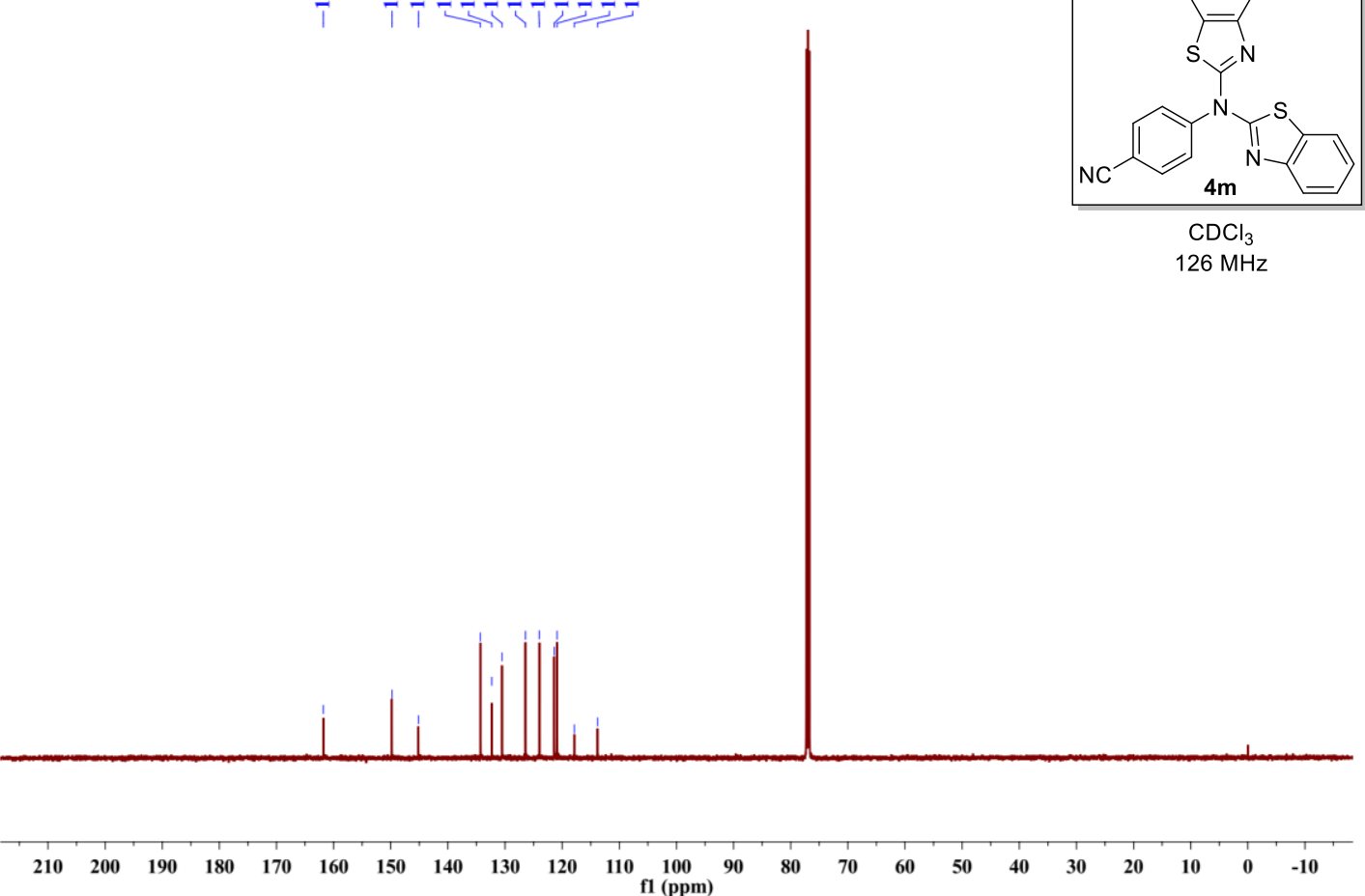


${ }^{1} \mathrm{H}$ NMR spectrum for 40

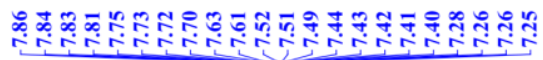

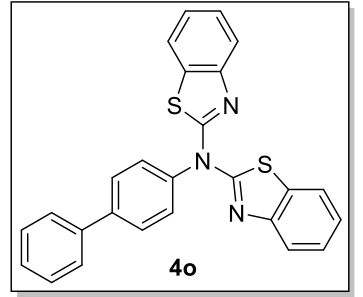

$\mathrm{CDCl}_{3}$

$500 \mathrm{MHz}$

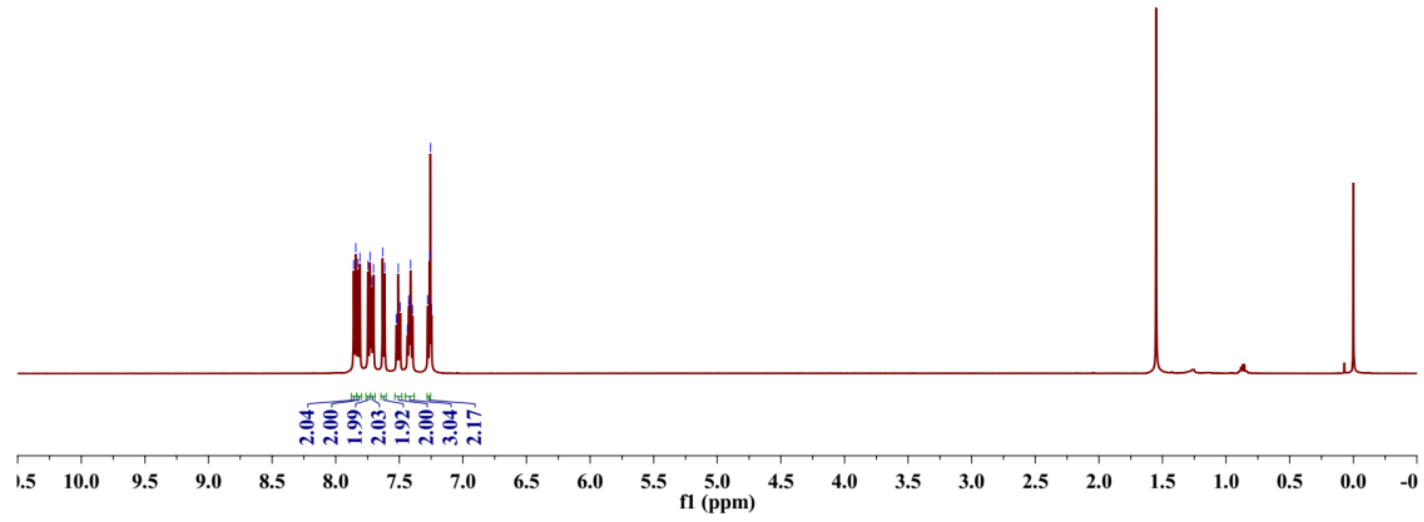

$>{ }^{13} \mathrm{C}$ NMR spectrum for 40

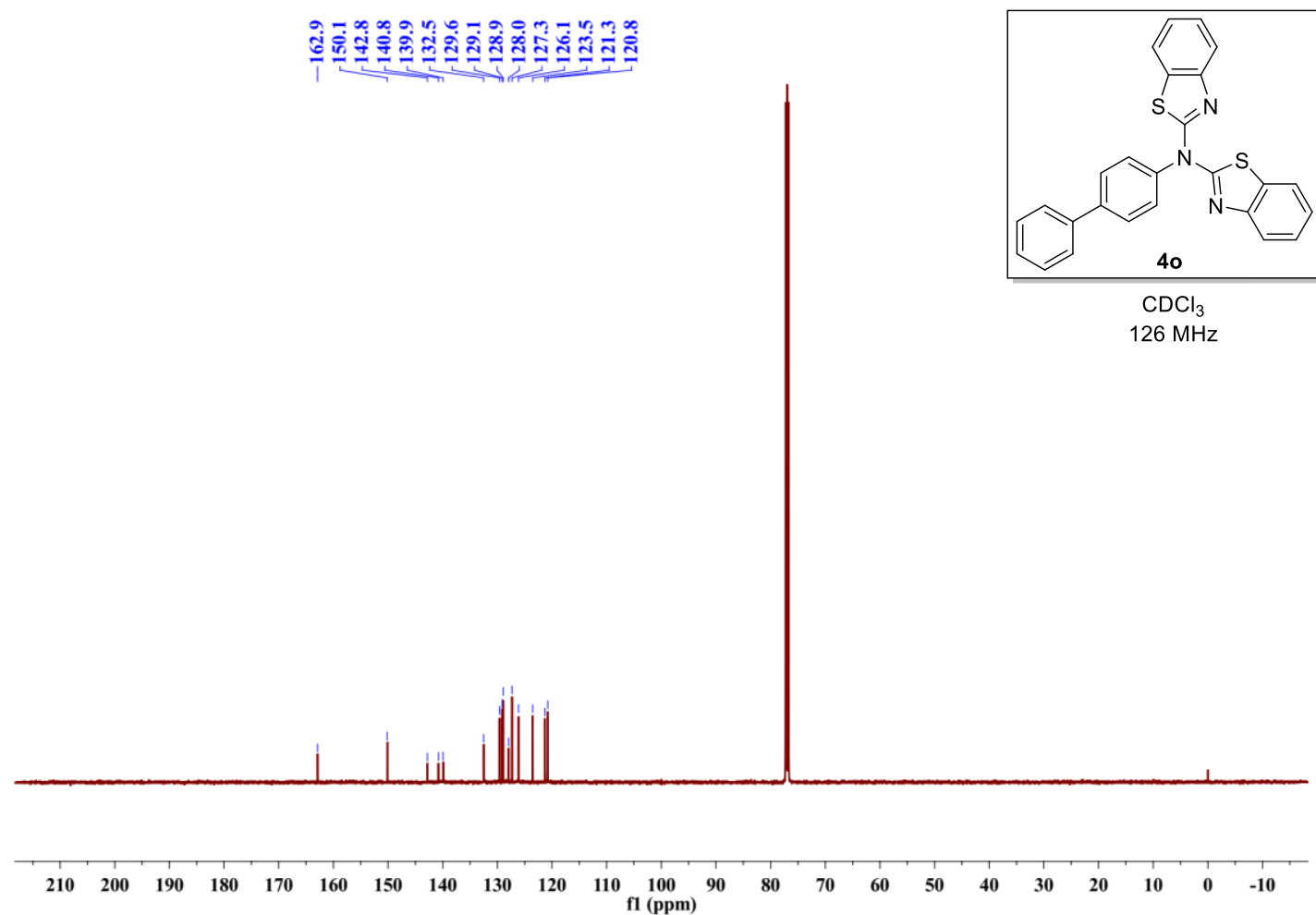


${ }^{1} \mathrm{H}$ NMR spectrum for $\mathbf{4 p}$

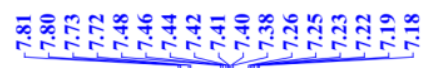
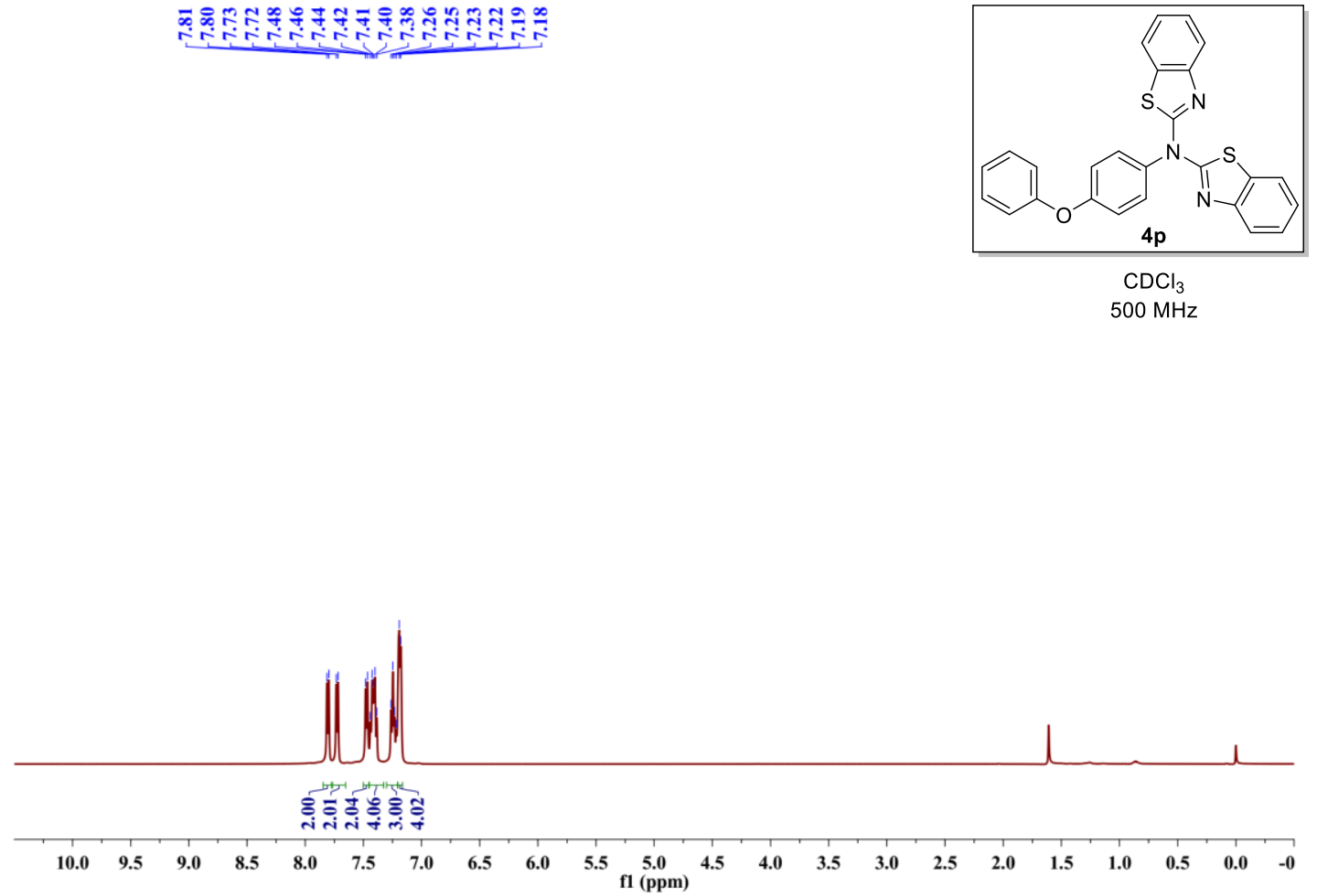

$>{ }^{13} \mathrm{C}$ NMR spectrum for $\mathbf{4 p}$

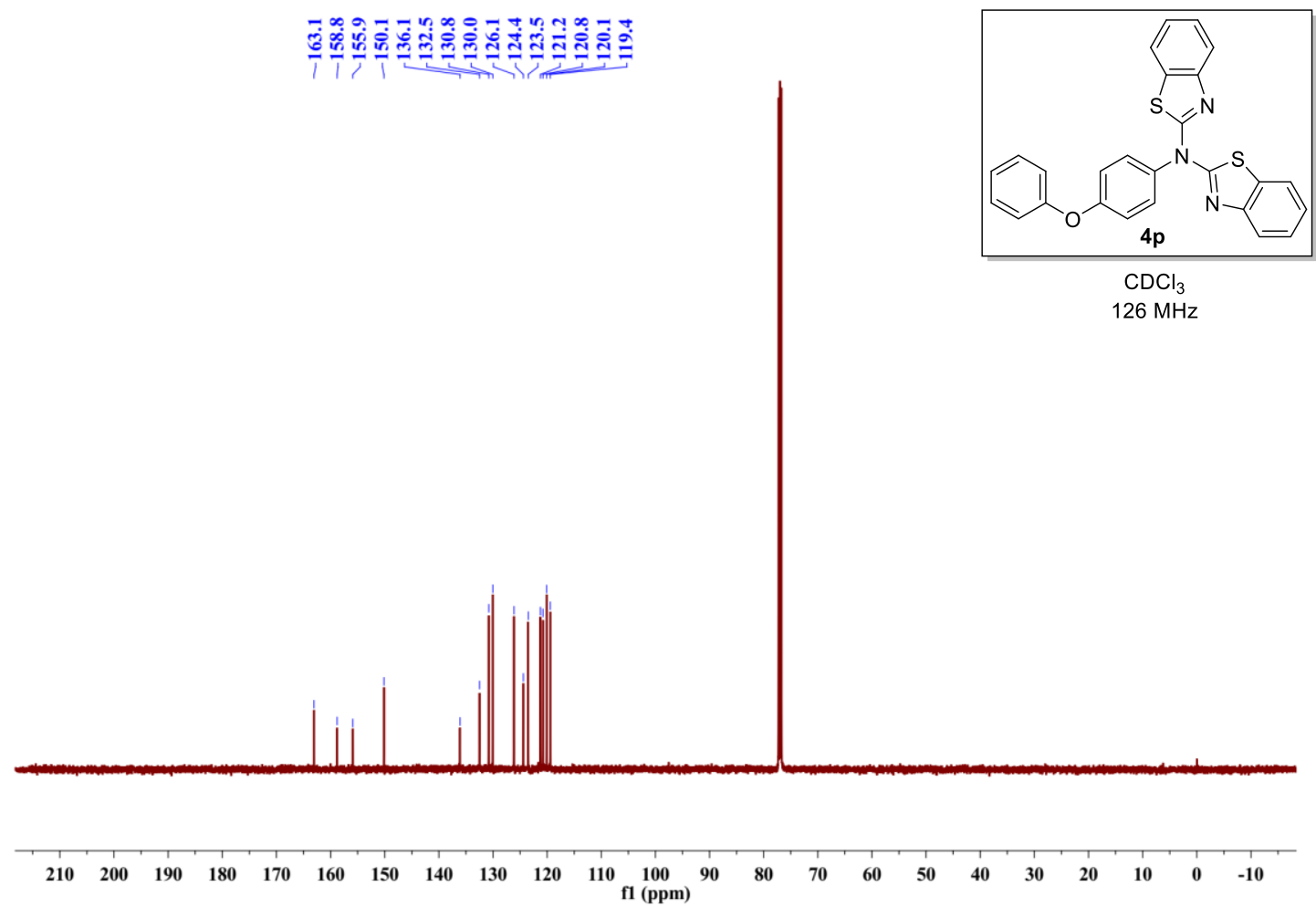


${ }^{1} \mathrm{H}$ NMR spectrum for $\mathbf{4 q}$

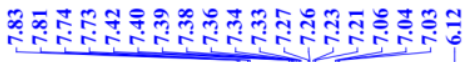

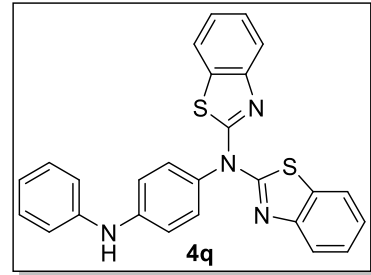

$\mathrm{CDCl}_{3}$ $500 \mathrm{MHz}$

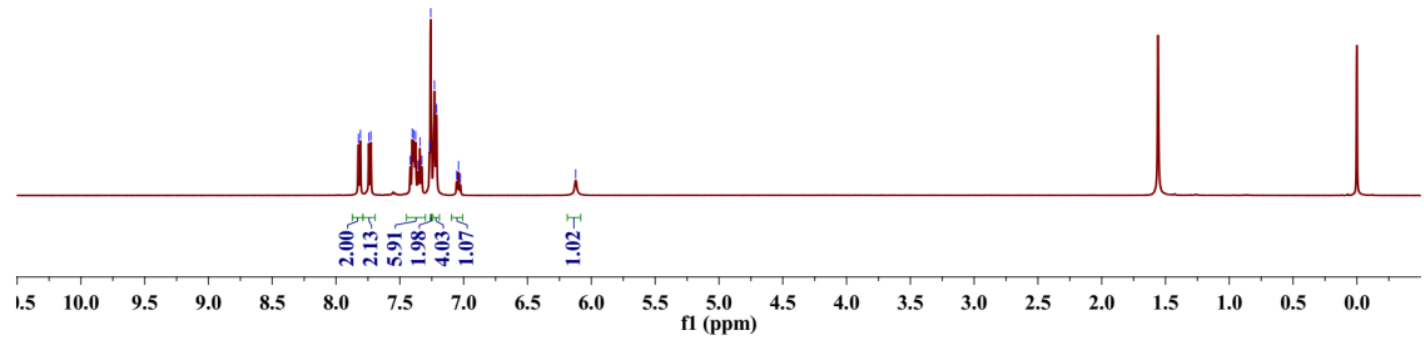

$>{ }^{13} \mathrm{C}$ NMR spectrum for $\mathbf{4 q}$

它

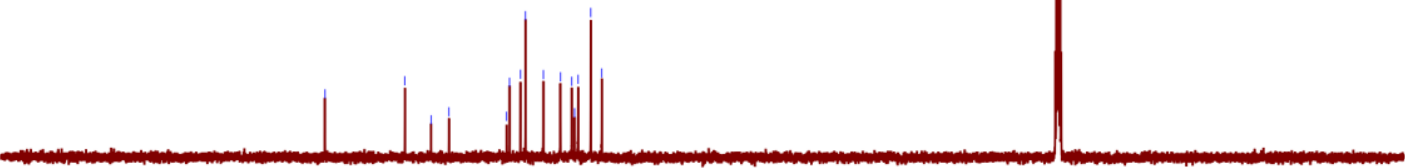

$\begin{array}{lllllllllllllllllllllll}210 & 200 & 190 & 180 & 170 & 160 & 150 & 140 & 130 & 120 & 110 & 100 & 90 & 80 & 70 & 60 & 50 & 40 & 30 & 20 & 10 & 0 & -10\end{array}$ 
${ }^{1} \mathrm{H}$ NMR spectrum for $\mathbf{4 r}$

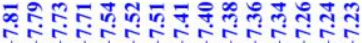

$\stackrel{\text { i }}{\text { ? }}$

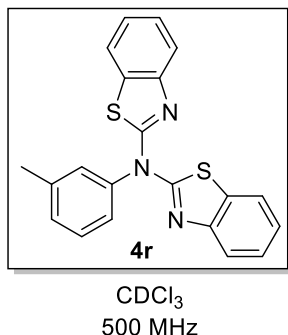

$500 \mathrm{MHz}$

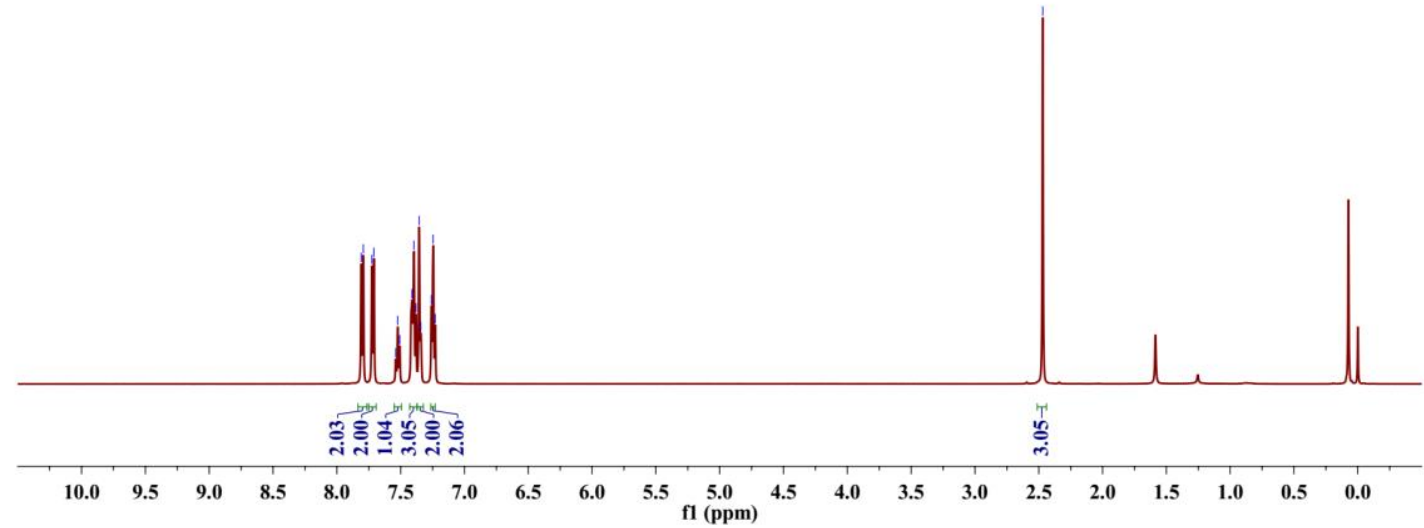

${ }^{13} \mathrm{C}$ NMR spectrum for $\mathbf{4 r}$

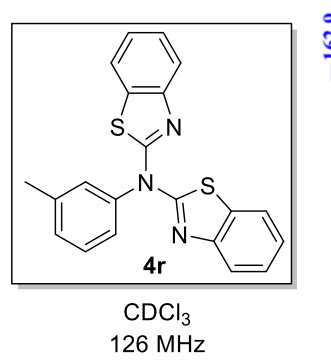

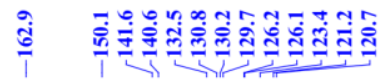

$\stackrel{4}{2}$

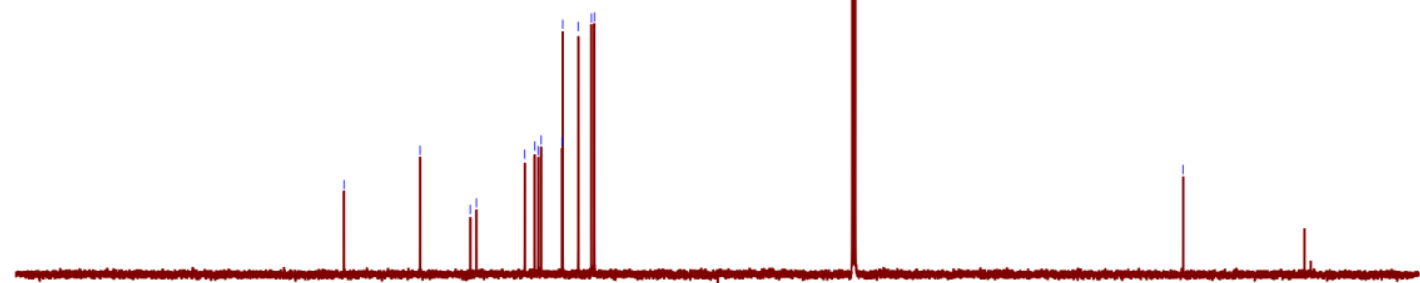

$\begin{array}{llllllllllllllllllllllll}210 & 200 & 190 & 180 & 170 & 160 & 150 & 140 & 130 & 120 & 110 & 100 & 90 & 80 & 70 & 60 & 50 & 40 & 30 & 20 & 10 & 0 & -10 & \end{array}$ 
${ }^{1} \mathrm{H}$ NMR spectrum for $\mathbf{4 s}$

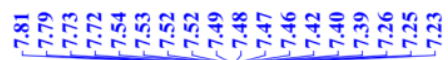

जे

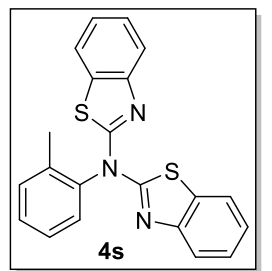

$\mathrm{CDCl}_{3}$ $500 \mathrm{MHz}$

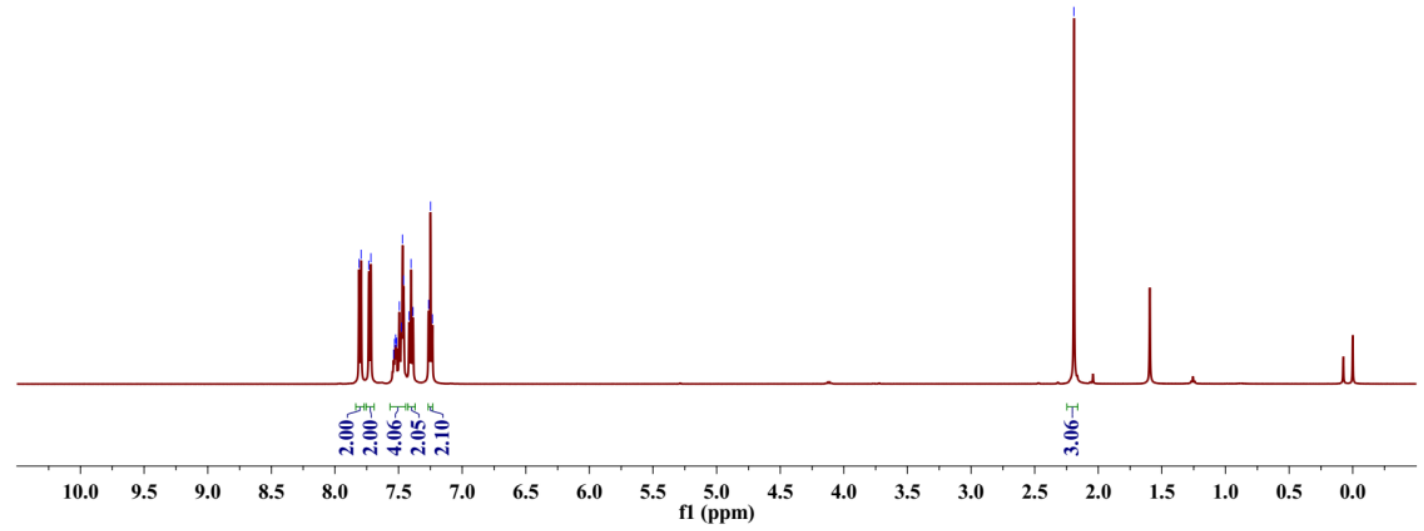

$>{ }^{13} \mathrm{C}$ NMR spectrum for $4 \mathrm{~s}$

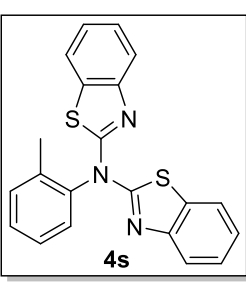

สี

$\stackrel{n}{7}$

$\mathrm{CDCl}_{3}$

$126 \mathrm{MHz}$

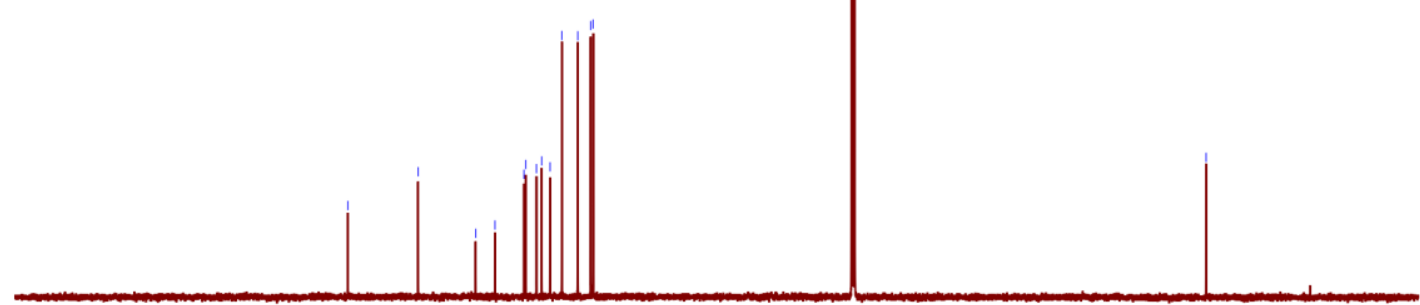

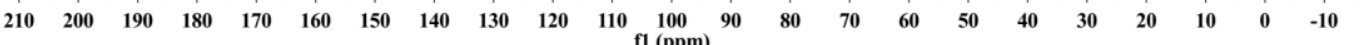


${ }^{1} \mathrm{H}$ NMR spectrum for $\mathbf{4 t}$

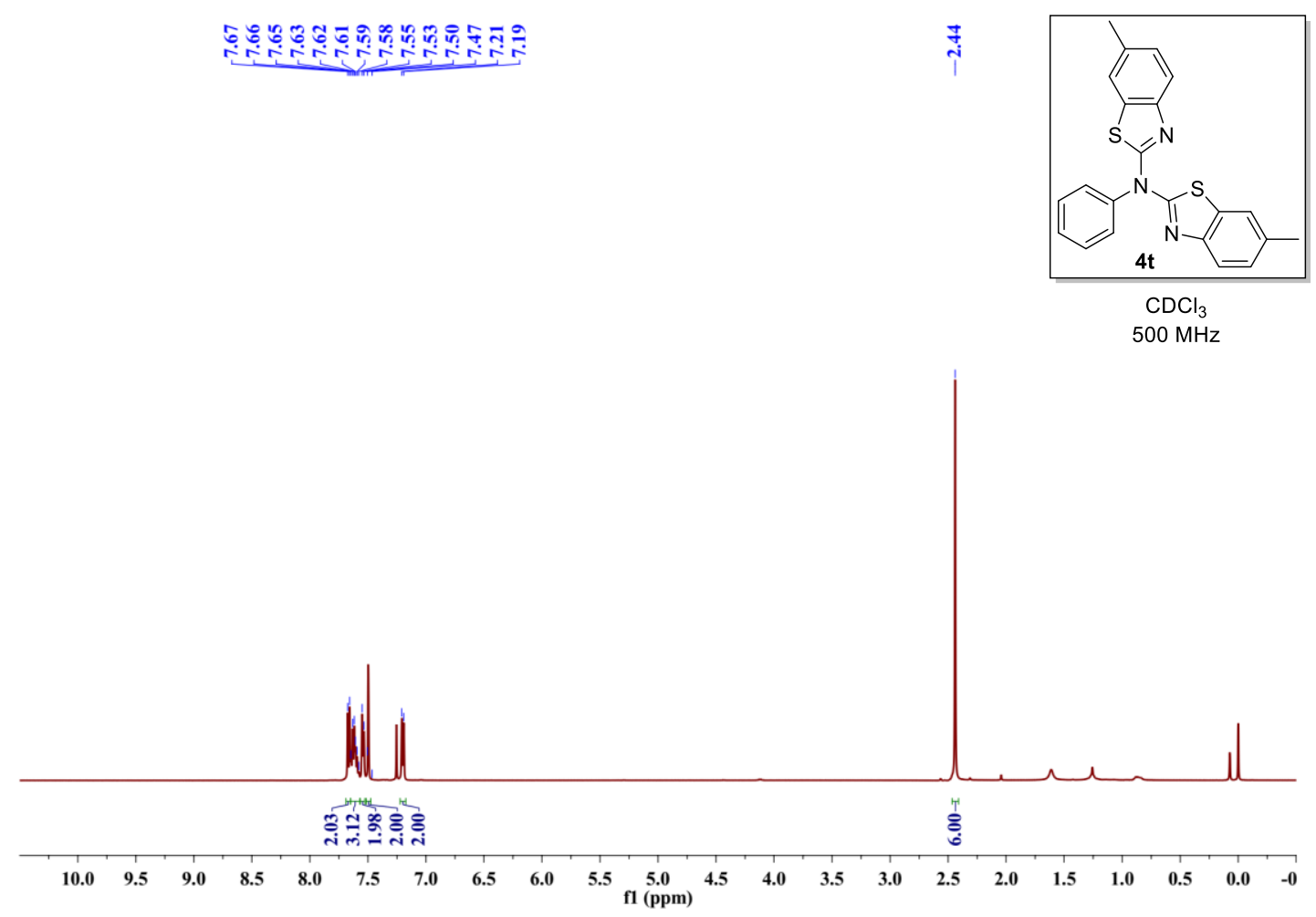

$>{ }^{13} \mathrm{C}$ NMR spectrum for $\mathbf{4 t}$

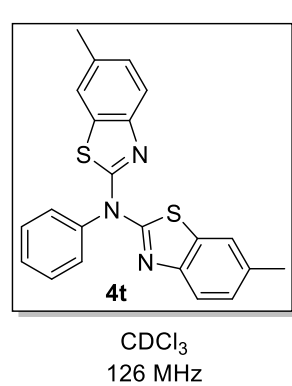

$\overline{\mathrm{i}} \quad \overline{\mathrm{s}}$

$\stackrel{n}{i}$

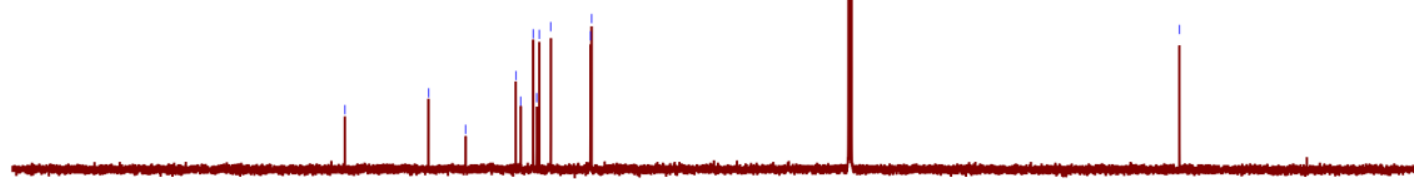

$\begin{array}{lllllllllllllllllllllll}210 & 200 & 190 & 180 & 170 & 160 & 150 & 140 & 130 & 120 & 110 & 100 & 90 & 80 & 70 & 60 & 50 & 40 & 30 & 20 & 10 & 0 & -10\end{array}$ 
${ }^{1} \mathrm{H}$ NMR spectrum for $\mathbf{4 u}$

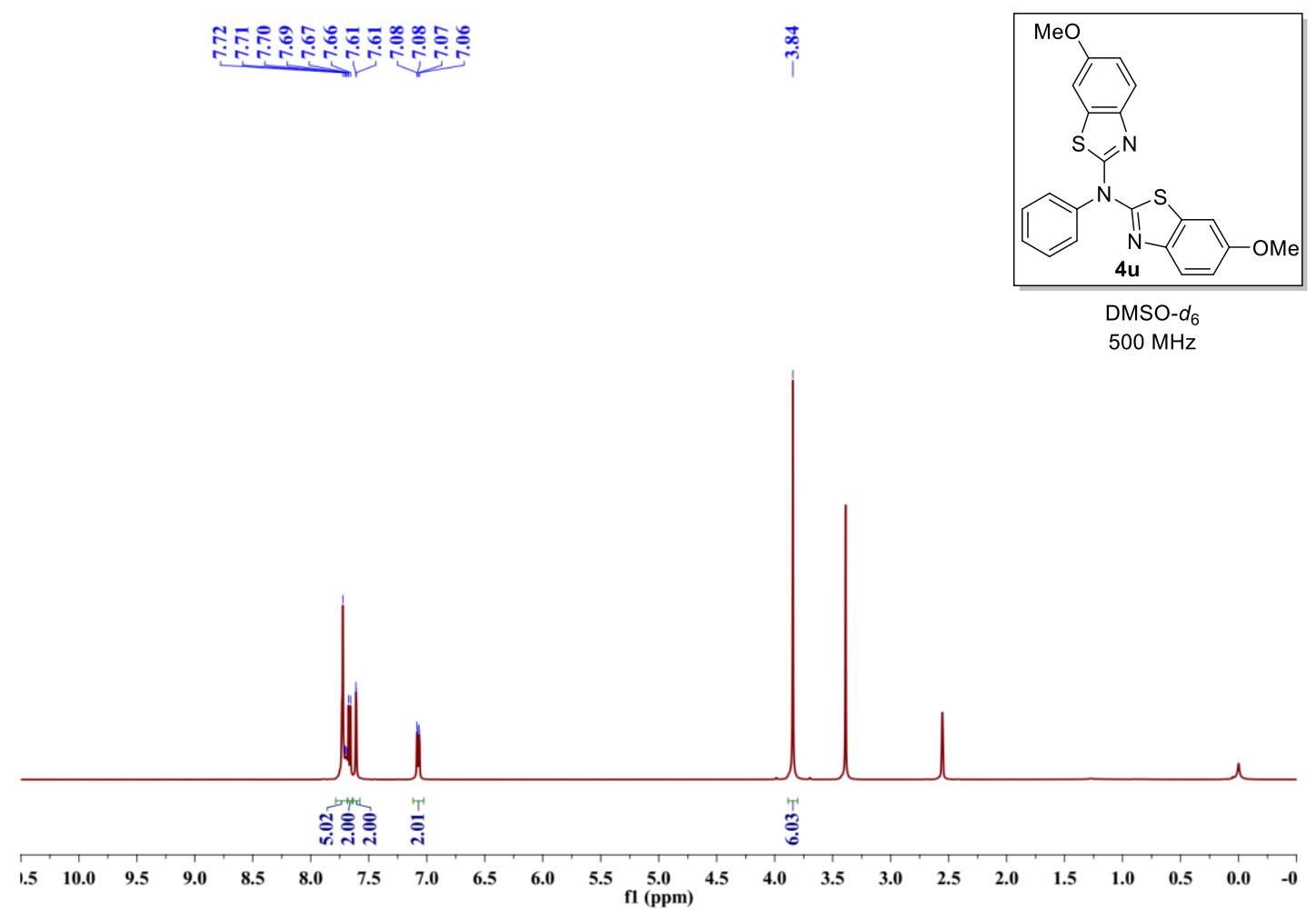

$>{ }^{13} \mathrm{C}$ NMR spectrum for $\mathbf{4 u}$

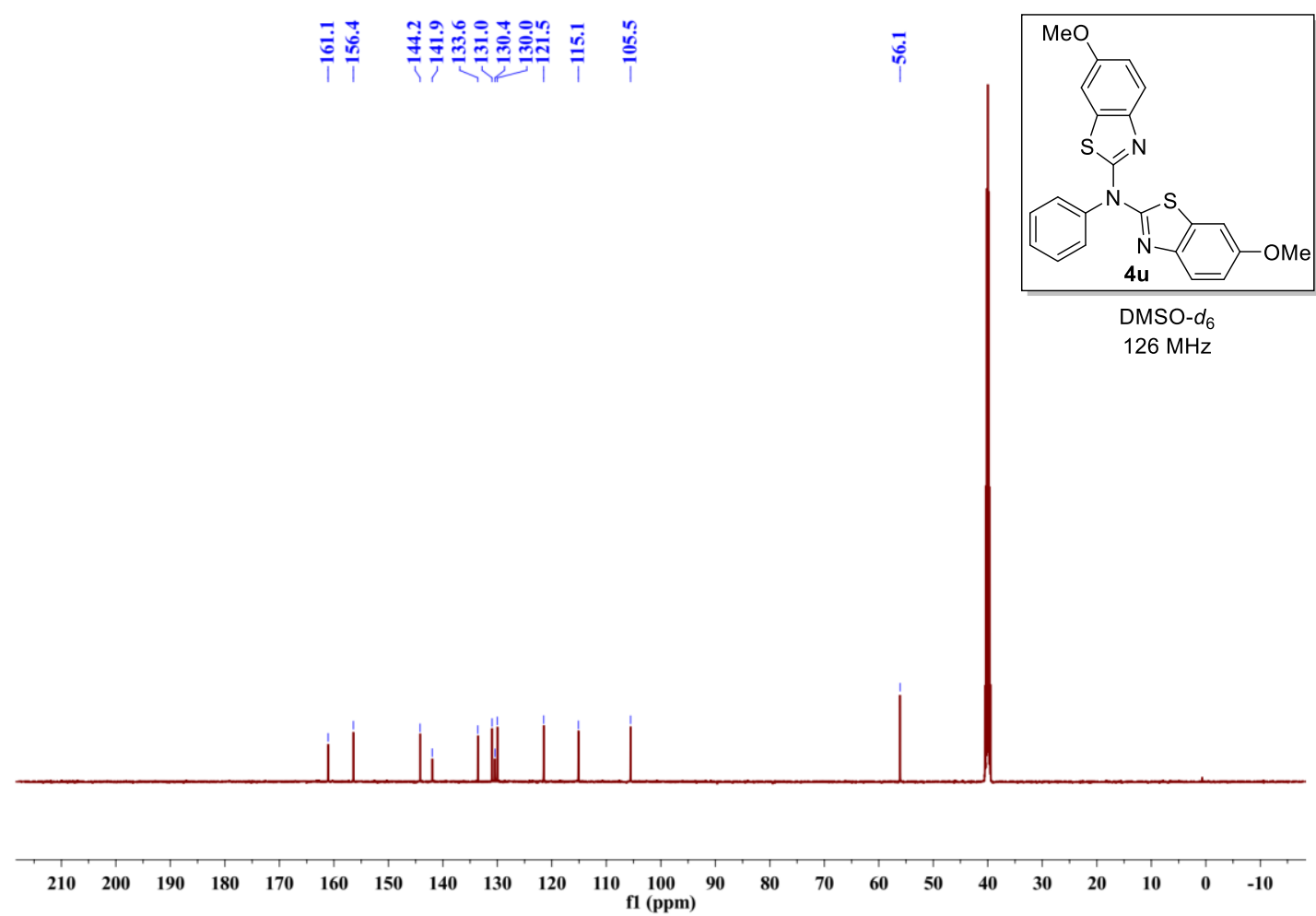


${ }^{1} \mathrm{H}$ NMR spectrum for $\mathbf{4 v}$

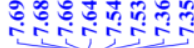
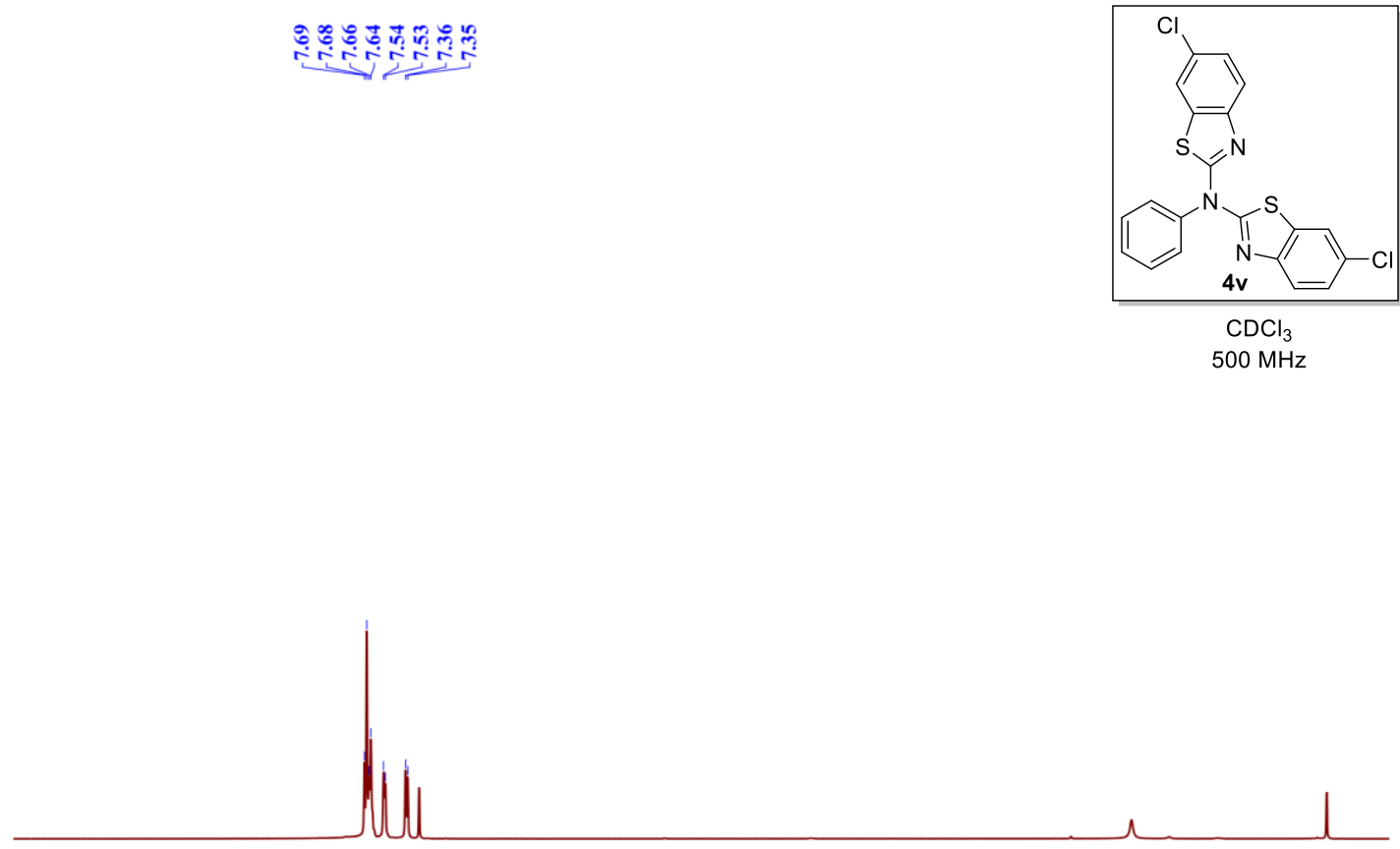

30

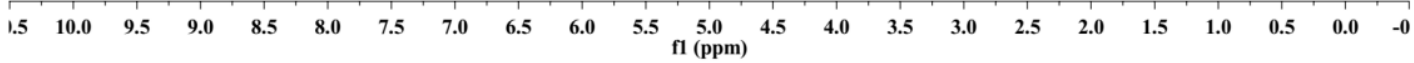

$>{ }^{13} \mathrm{C}$ NMR spectrum for $\mathbf{4 v}$

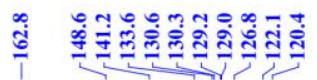

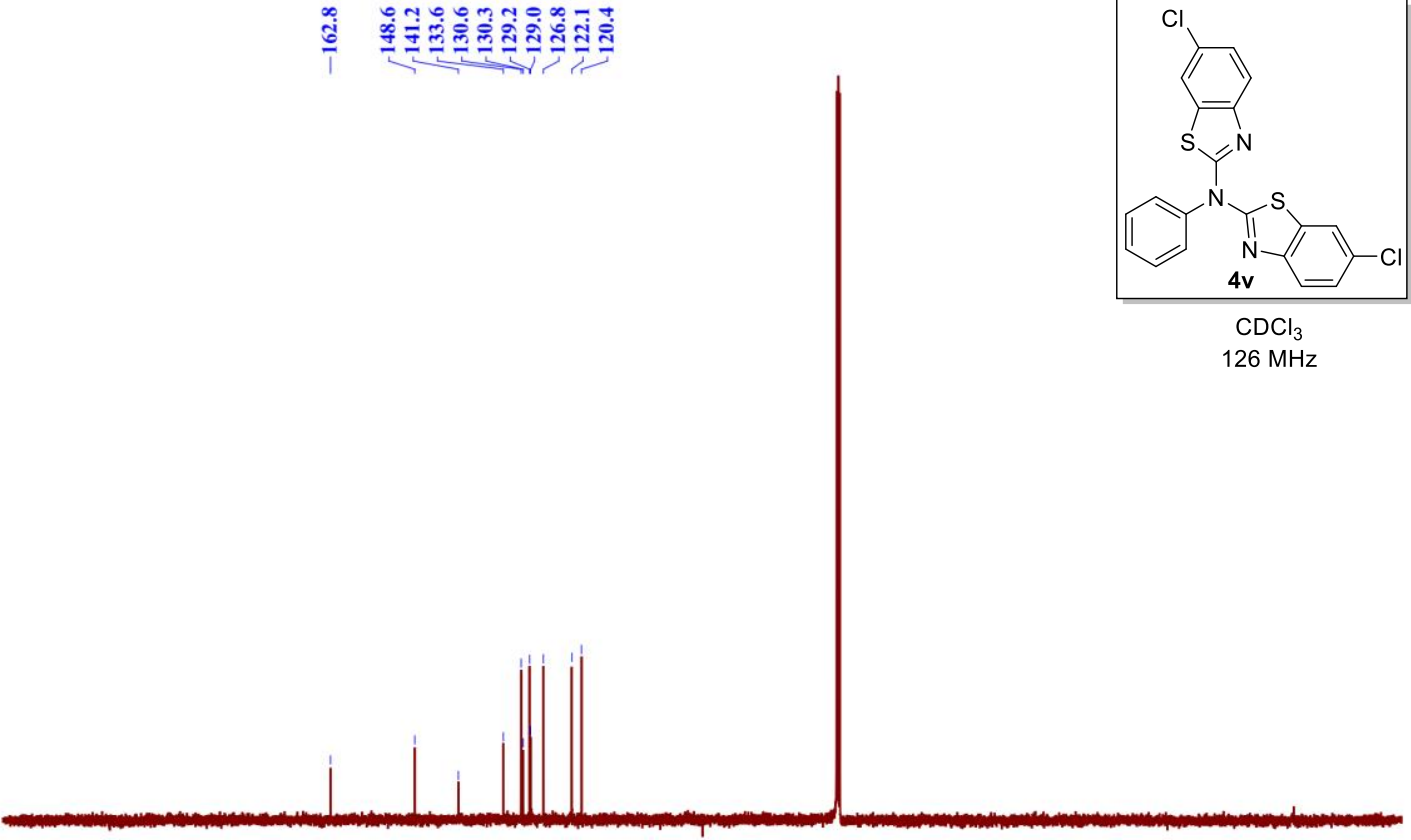

$\begin{array}{lllllllllllllllllllllll}210 & 200 & 190 & 180 & 170 & 160 & 150 & 140 & 130 & 120 & 110 & 100 & 90 & 80 & 70 & 60 & 50 & 40 & 30 & 20 & 10 & 0 & -10\end{array}$ 
${ }^{1} \mathrm{H}$ NMR spectrum for $\mathbf{4 w}$

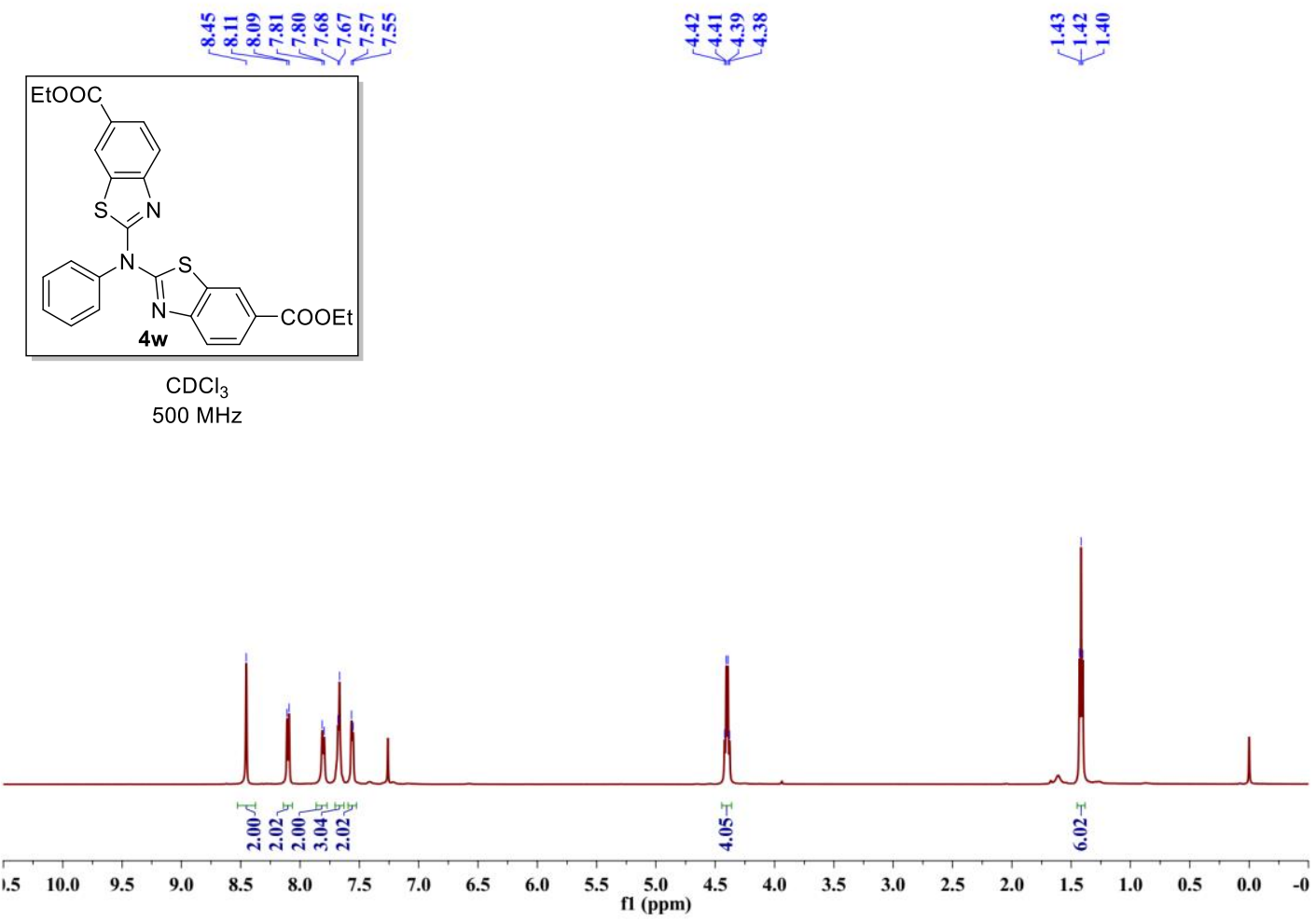

$>{ }^{13} \mathrm{C}$ NMR spectrum for $\mathbf{4 w}$

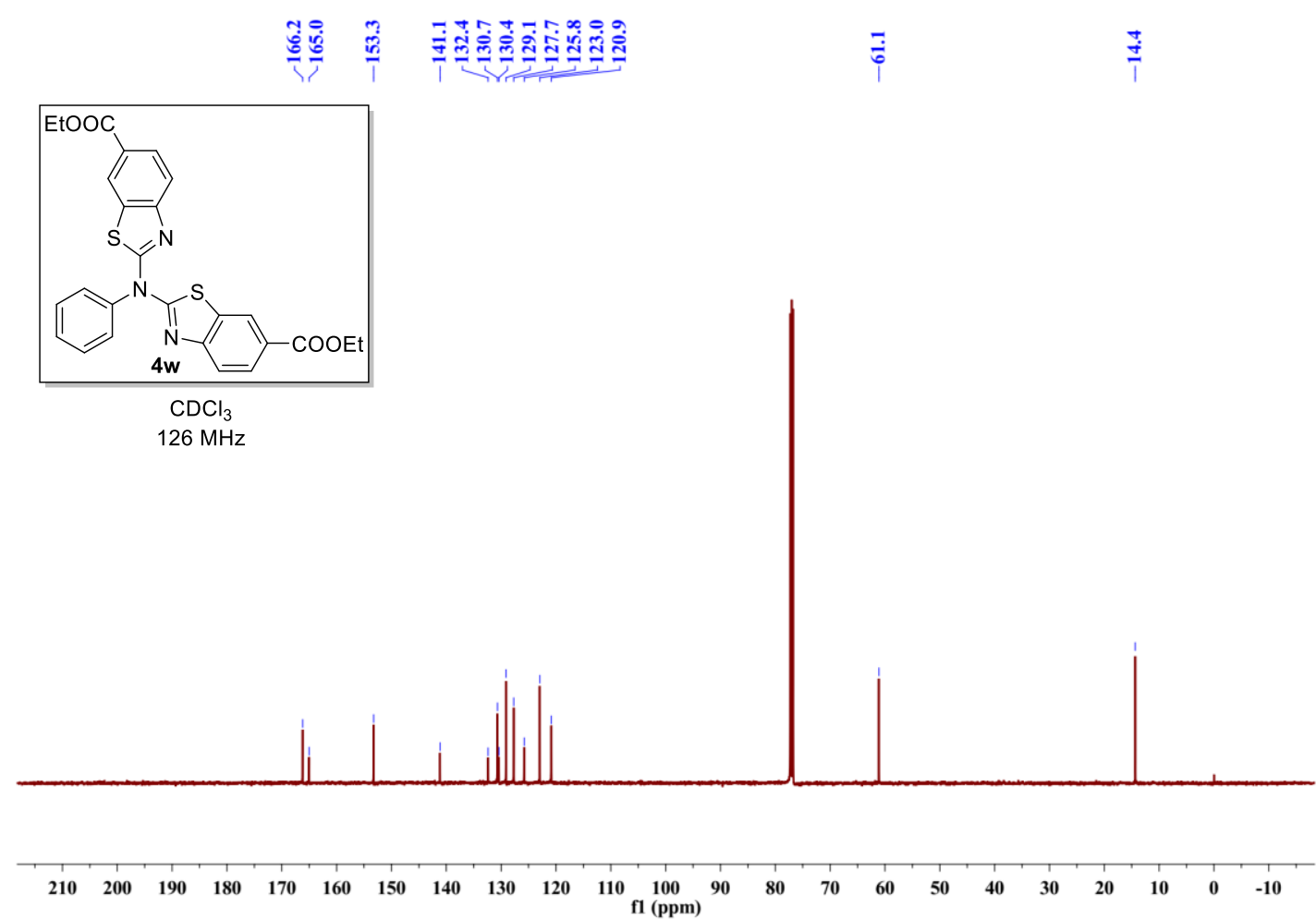


${ }^{1} \mathrm{H}$ NMR spectrum for $\mathbf{4 x}$

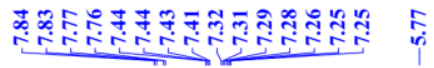

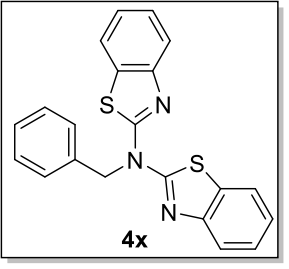

$\mathrm{CDCl}_{3}$ $500 \mathrm{MHz}$

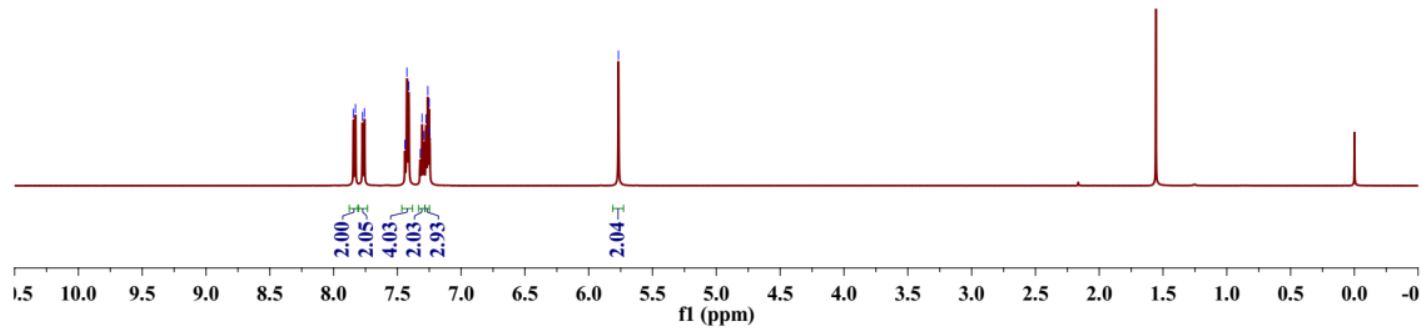

$>{ }^{13} \mathrm{C}$ NMR spectrum for $\mathbf{4} \mathbf{x}$

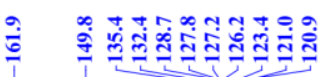

$\underset{i}{\infty}$

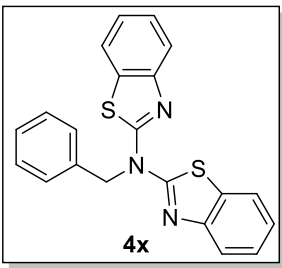

$\mathrm{CDCl}_{3}$ $126 \mathrm{MHz}$

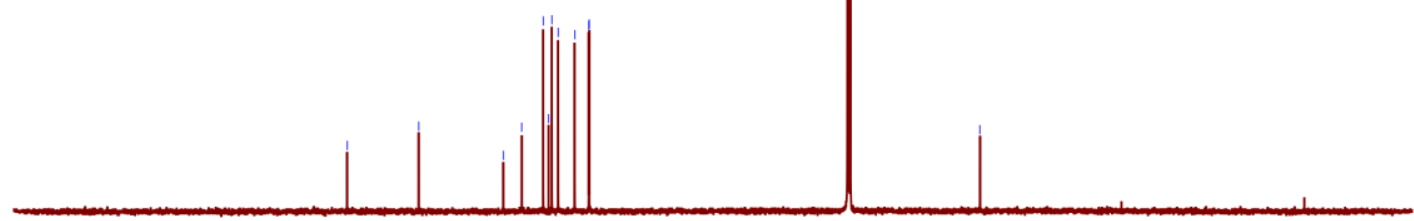

$\begin{array}{llllllllllllllllllllllll}210 & 200 & 190 & 180 & 170 & 160 & 150 & 140 & 130 & 120 & 110 & 100 & 90 & 80 & 70 & 60 & 50 & 40 & 30 & 20 & 10 & 0 & -10\end{array}$ 
${ }^{1} \mathrm{H}$ NMR spectrum for $\mathbf{4 y}$
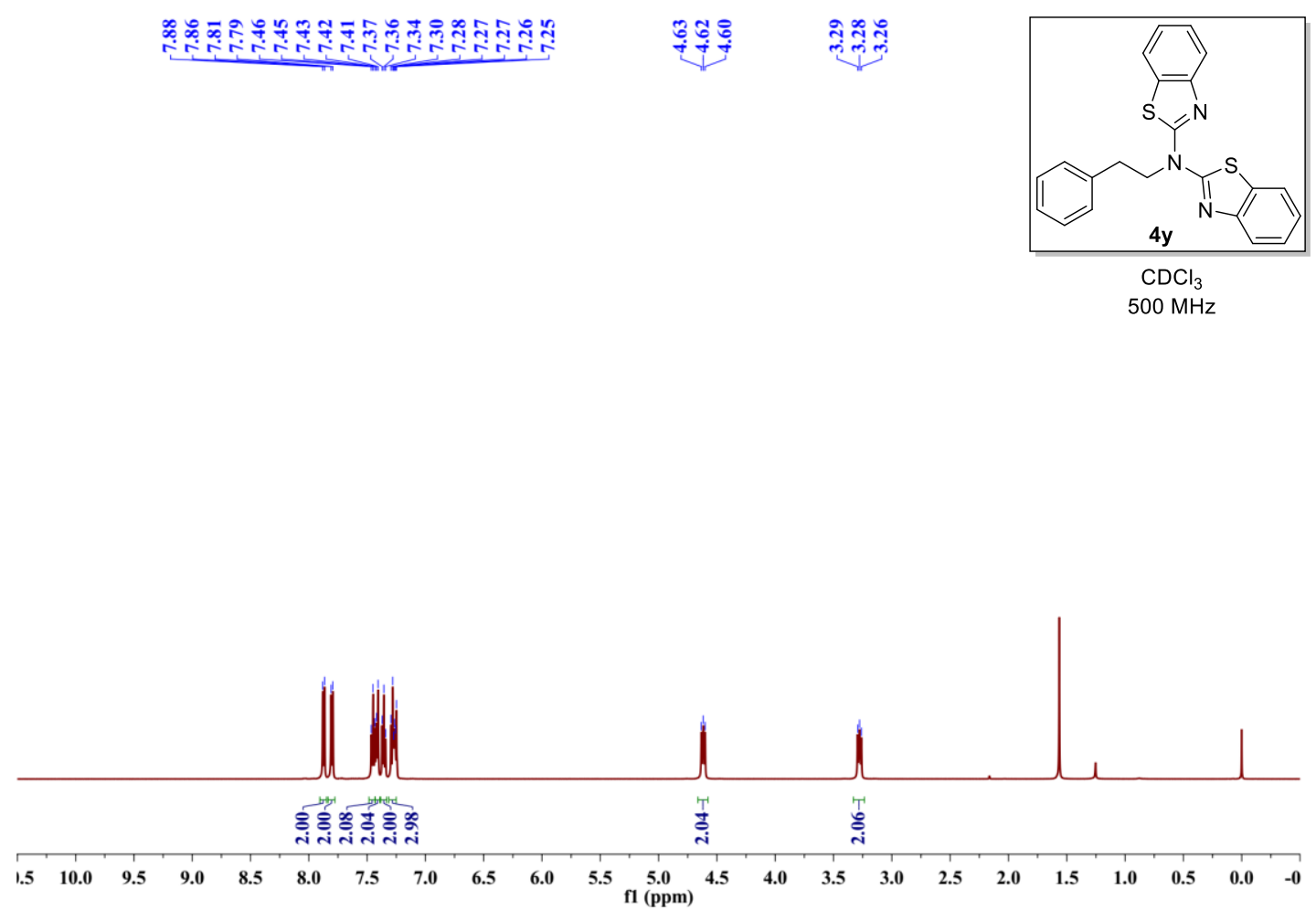

$>{ }^{13} \mathrm{C}$ NMR spectrum for $\mathbf{4 y}$

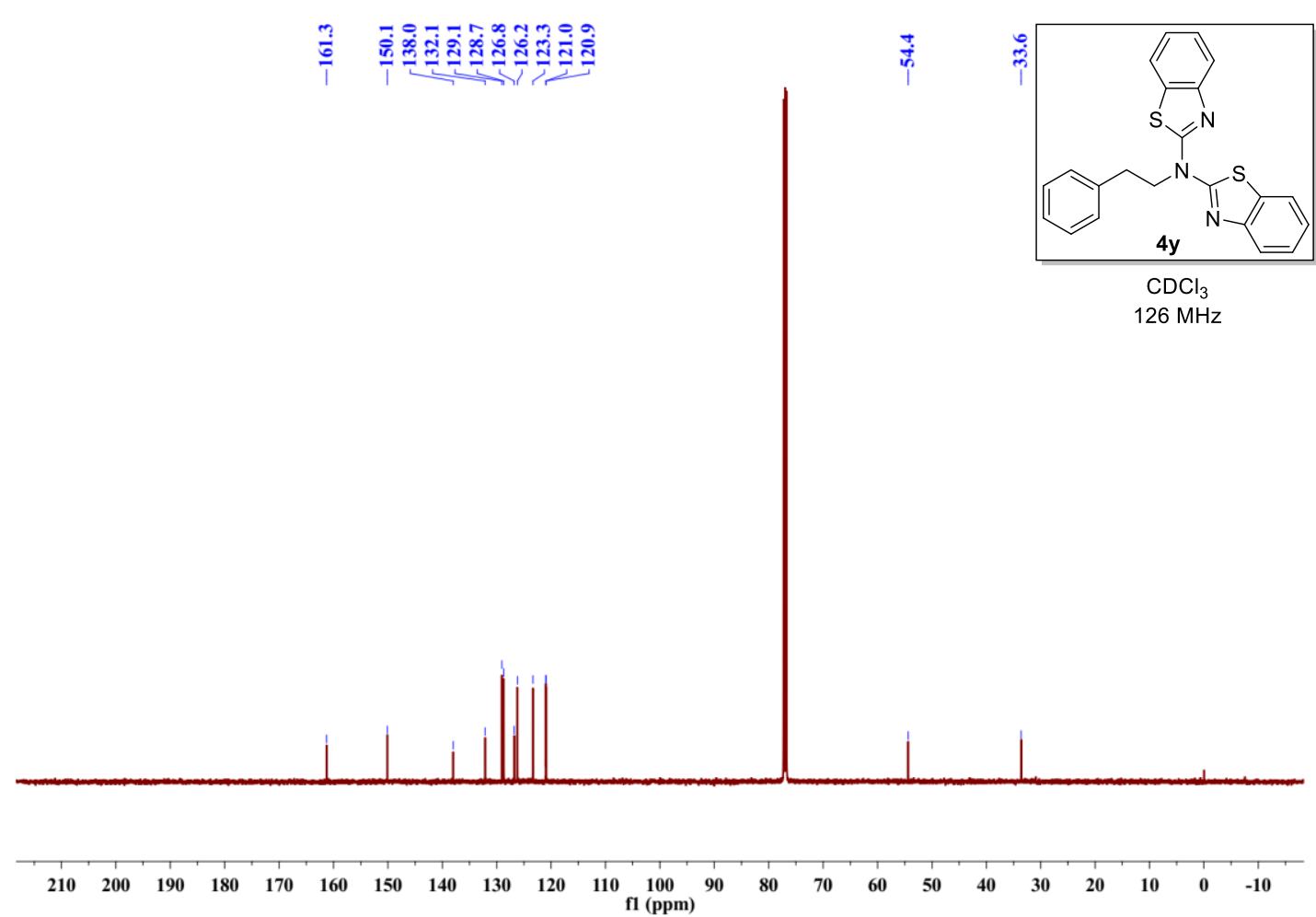


${ }^{1} \mathrm{H}$ NMR spectrum for $\mathbf{4 z}$

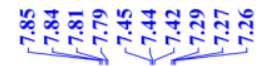
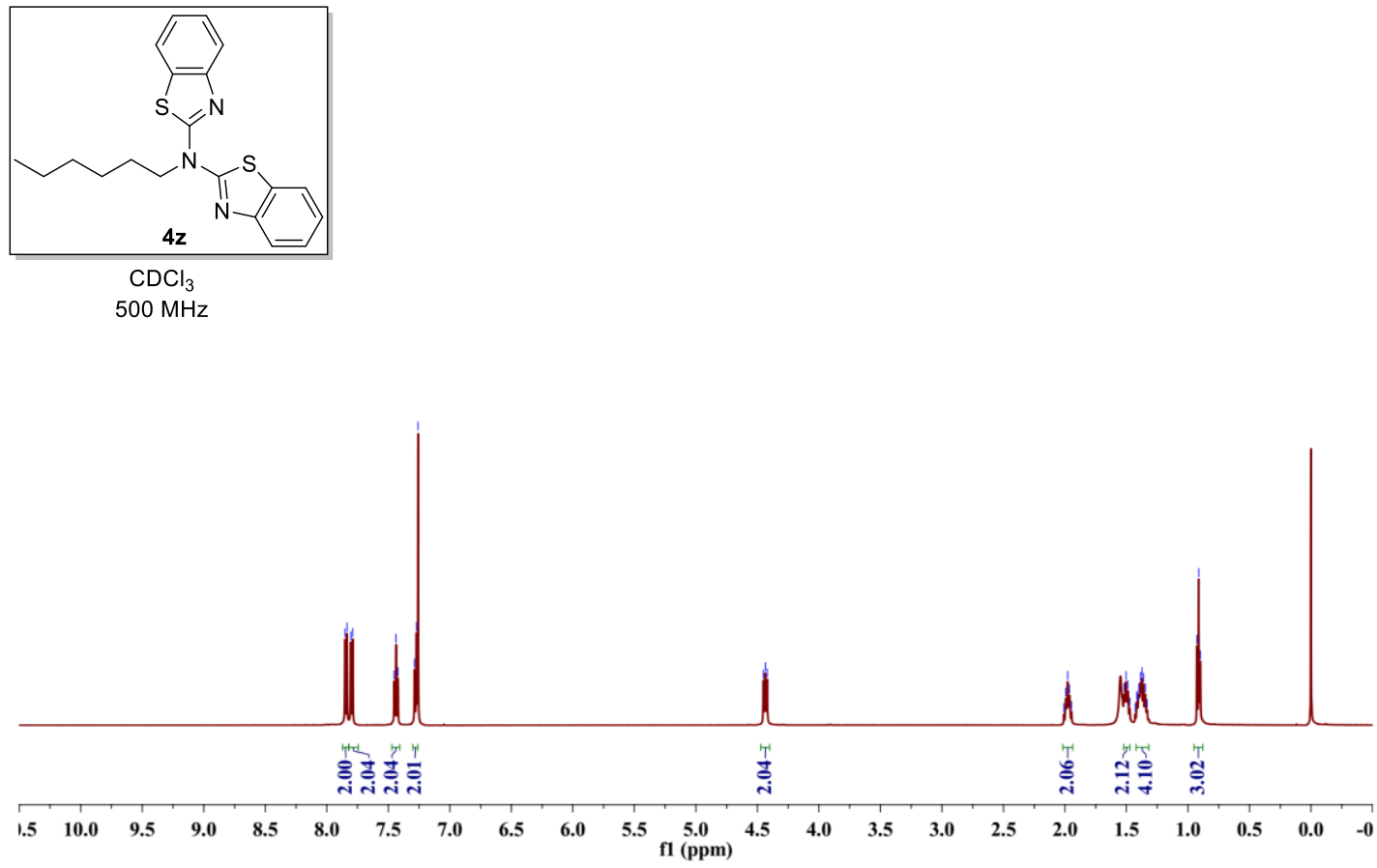

$>{ }^{13} \mathrm{C}$ NMR spectrum for $\mathbf{4 z}$

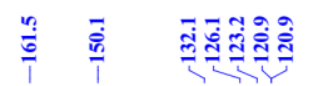

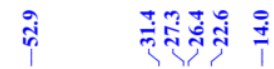

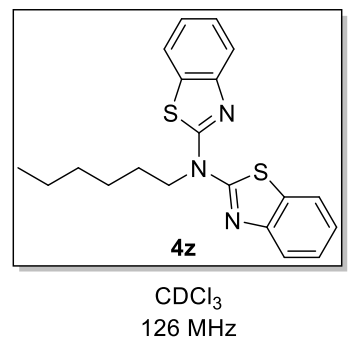

$\begin{array}{lllllllllllllllllllllll}210 & 200 & 190 & 180 & 170 & 160 & 150 & 140 & 130 & 120 & 110 & \begin{array}{c}100 \\ \mathrm{fl}(\mathrm{ppm})\end{array} & 90 & 80 & 70 & 60 & 50 & 40 & 30 & 20 & 10 & 0 & -10\end{array}$ 